\title{
MOLECULAR CONTROL OF \\ ENDOTHELIAL CELL POLARIZATION AND \\ TUBE FORMATION BY RAS SUPERFAMILY GTPASES
}

A Dissertation
presented to
the Faculty of the Graduate School
at the University of Missouri-Columbia
In Partial Fulfillment
of the Requirements for the Degree
Doctor of Philosophy
PIETER R. NORDEN
Dr. George E. Davis, Dissertation Supervisor

December 2015 
The undersigned, appointed by the dean of the Graduate School, have examined the dissertation entitled

\section{MOLECULAR CONTROL OF ENDOTHELIAL CELL POLARIZATION AND TUBE FORMATION BY RAS SUPERFAMILY GTPASES}

presented by Pieter R. Norden, a candidate for the degree of doctor of philosophy and hereby certify that, in their opinion, it is worthy of acceptance.

Dr. George E. Davis

Dr. Anand Chandrasekhar

Dr. William Durante

Dr. Luis Martinez-Lemus

Dr. Greg Sowa 


\section{ACKNOWLEDGEMENTS}

I would like to first and foremost thank my mentor Dr. George E. Davis for his incredible support and encouragement during the pursuit of my doctorate. It has been a great privilege to learn and develop in an inspiring and challenging environment under the mentorship of someone who is an exceptional representation of passion, innovation and integrity within the field of scientific investigation.

I would also like to thank my committee members Drs. Anand Chandrasekhar, William Durante, Luis Martinez-Lemus and Greg Sowa for their input and assistance in my training, as well as the faculty and staff of the Genetics Area Program, the department of Medical Pharmacology and Physiology, and the Life Sciences Fellowship Program. I am incredibly grateful for their assistance and support in my development as a young investigator.

Additionally, I would like to thank all of the past and present lab members of the Davis lab that I have had the privilege of working with. I am thankful to have learned and developed with the help of many talented, inspiring individuals and to be able to create long lasting relationships. I know I would not be where I am today without their time, support and guidance given to me.

Finally, I would like to thank my family, Steve, Jean, Billy, Mara and Charlie Norden and especially my wife Amy for their unconditional love and support. It is because of this, their sacrifices and belief in me that I have been able to pursue this degree. 


\section{TABLE OF CONTENTS}

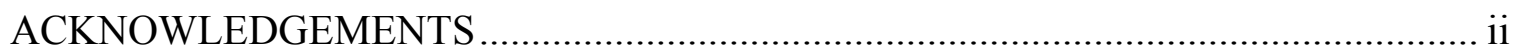

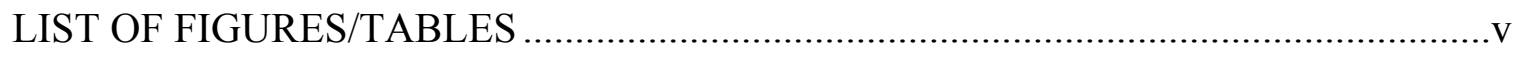

CHAPTERS:

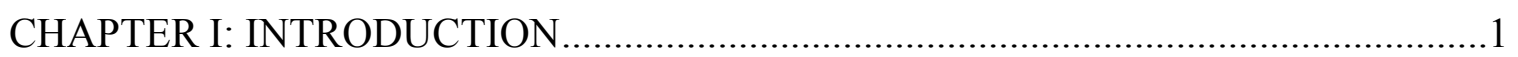

An overview of the vasculature ........................................................... 1

Regulation of asymmetric cell polarity during lumen formation ..................2

A comparison of endothelial and epithelial de novo lumen formation .........4

The endothelial lumen signaling cascade ................................................

An overview of key signaling mechanisms ................................ 7

Integrin and ECM interaction during EC tubulogenesis.................8

The role of hematopoietic cytokines, FGF-2 and VEGF signaling

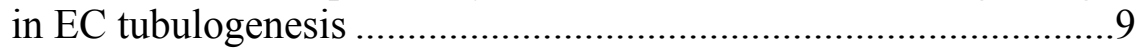

MT1-MMP proteolytic activity is required for the formation of vascular guidance tunnels and activation of $\mathrm{Cdc} 42$ during EC tubulogenesis

Src family kinase and protein kinase $\mathrm{C}$ activity in controlling EC tubulogenesis

The role of Ras superfamily GTPase regulation of endothelial lumen formation and vessel stabilization.

An overview of Ras superfamily GTPases ................................. 14

Rho-family GTPase regulation of cytoskeletal organization, cell

polarity and lumen formation.....

Ras superfamily members implicated in endothelial morphogenesis

and vessel integrity .......................................................... 18

Regulation of membrane trafficking events in lumen formation ..............22

Mechanisms of exocytic vesicle fusion controlled by GTPase activity ....25

The importance of integrating in vitro and in vivo models in investigating

mechanisms of vascular development and regulation ..........................28

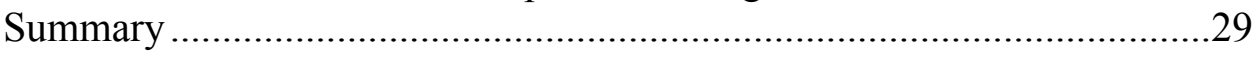

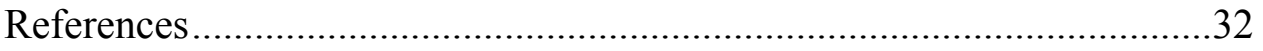


CHAPTER II: CDC42 AND K-RAS CONTROL ENDOTHELIAL TUBULOGENESIS AND APICAL POLARIZATION: NEGATIVE REGULATION BY ARHGAP31 AND RASA1 ...............................................................

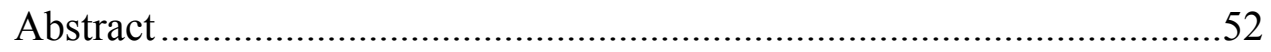

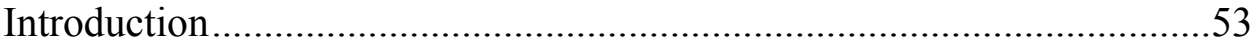

Materials and Methods.........................................................................55

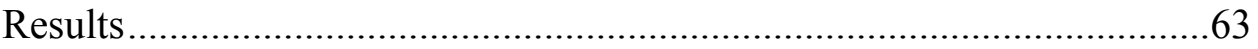

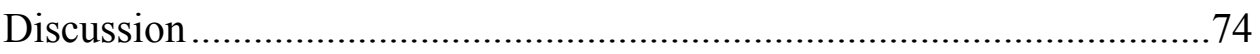

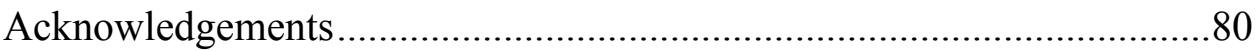

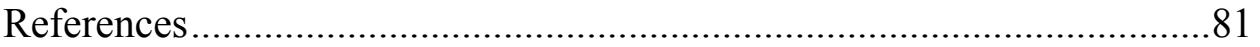

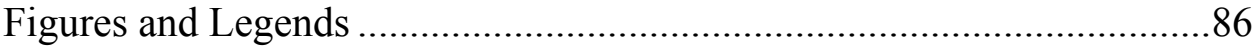

CHAPTER III: RAB AND RAL GTPASES CONTROL ENDOTHELIAL CELL TUBULOGENESIS DURING POLARIZED TRAFFICKING AND FUSION OF CAVEOLIN-1-LABELED PINOCYTIC VACUOLES TO GENERATE THE APICAL MEMBRANE SURFACE .........................100

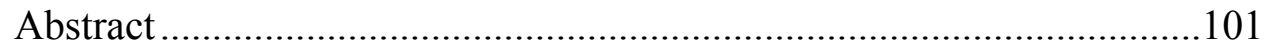

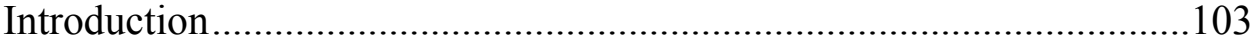

Materials and Methods......................................................................106

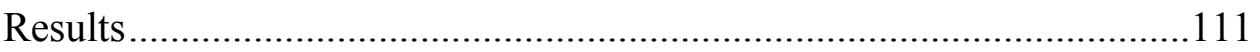

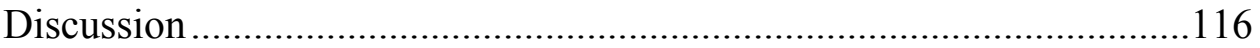

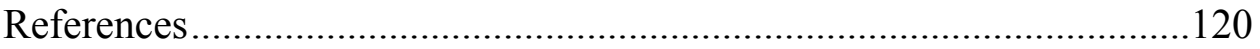

Figures and Legends ………………………………….................125

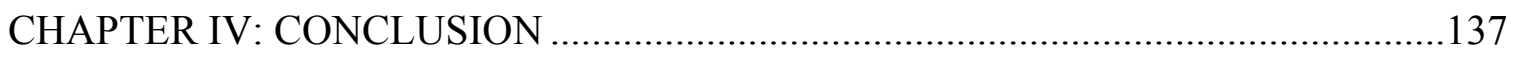

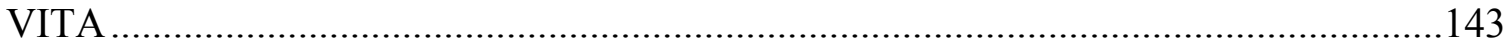




\section{LIST OF FIGURES/TABLES}

Figure

Page

1.1 The Ras superfamily of small GTPases .21

1.2 Cdc42-associated molecular signaling complexes control EC lumen formation and tubulogenesis in 3D collagen matrices

1.3 Localization and function of Rab GTPases in an epithelial cell .21

1.4 Polarized trafficking and fusion of pinocytic intracellular vacuoles and membrane vesicles creates the apical luminal area during endothelial tubulogenesis

2.1 Identification of key small GTPases that control EC tubulogenesis in 3D matrices

2.2 Signaling events that characterize EC tubulogenesis over time in 3D collagen matrices

2.3 Apical polarization of Cdc42, Rac1 and acetylated tubulin during EC tubulogenesis: Membrane trafficking of vacuoles along microtubule tracks toward the apical surface directs EC lumen formation in 3D matrices.

2.4 EC tubulogenesis is negatively regulated by the GAPs, Arhgap31 and Rasa1, and is positively regulated by Arhgap29

2.5 Increased Cdc42, Rac, and Ras activity via siRNA suppression of Arhgap31, Rasa1 or both stimulates key signaling pathways and molecules that control EC tubulogenesis

2.6 IQGAP1, MRCK $\beta$, Rasip1, and beta-Pix are critical downstream effectors of small GTPase signaling that control EC tubulogenesis: Rasip1 controls EC tubulogenesis in vivo during mouse development and targets to the EC apical surface in vitro and in vivo

2.7 Cdc42, Rac1, Rac2, and k-Ras co-associate with $\alpha 2$ integrin, MT1-MMP and key GTPase effectors in lumen signaling complexes during EC tubulogenesis in 3D collagen matrices 
2.8 EC tubulogenesis in 3D collagen matrices is controlled by activation of a Cdc42-, Rac-, and k-Ras-dependent signaling cascade: A process that is antagonized by Arhgap31, Rasa1 and RhoA .93

S2.1 Apical Polarization of small GTPases and effectors that control EC tubulogenesis

S2.2 Cdc42- and Rac-dependent signaling affect tubulin modifications that control EC lumen formation and apical polarization: Key role of small GTPase effectors during EC tubulogenesis

S2.3 Small GTPase effectors are fundamental regulators of EC tubulogenesis in 3D matrices

3.1 Identification of key membrane trafficking regulators that control EC tubulogenesis in 3D matrices

3.2 Combined siRNA suppression of Rab GTPases and Caveolin-1 delineate functional differences during EC tubulogenesis

3.3 EC tubulogenesis requires differential regulation of membrane trafficking events by Rab8A, Rab11A, Rab27A and Caveolin-1 127

3.4 RalA and RalB are required for EC tubulogenesis in 3D matrices 128

3.5 Functional differences of small GTPase regulators of exocytosis and endocytosis are critical during EC tubulogenesis in 3D matrices

3.6 RalB is a critical regulator of EC tubulogenesis functionally distinct from Rab3A, Rab3B, Rab27A and RalA 130

T3.1 Generation of recombinant adenovirus constructs to assess subcellular localization and polarization of key regulators of EC tubulogenesis and membrane trafficking during EC lumen formation 131

3.7 Caveolin-1 targets to pinocytic intracellular vacuoles and the apical membrane surface during EC tubulogenesis in 3D matrices

3.8 RalA targets to pinocytic intracellular vacuoles and the apical membrane surface during EC tubulogenesis in 3D matrices

3.9 Src targets to pinocytic intracellular vacuoles and the subapical domain during EC tubulogenesis in 3D matrices 134 
3.10 Src and Rac activity regulate pinocytic intracellular vacuolization necessary for EC tubulogenesis whereas MT1-MMP activity regulates fusion of intracellular vacuoles downstream

3.11 Generation, apical trafficking and fusion of pinocytic intracellular vacuoles along modified tubulin tracks is controlled by Cdc42, Rac1, Rab and Ral GTPases in coordination with Src and Caveolin-1 during EC tubulogenesis

4.1 Key molecular mechanisms controlling EC tubulogenesis by Cdc42-, Rac-, k-Ras- and Rap1b- dependent signaling

4.2 Functional differences in GTPase signaling control separate events during EC tubulogenesis in coordination with key kinase, integrin and proteolytic activity 


\section{CHAPTER I}

\section{INTRODUCTION}

\section{An overview of the vasculature}

The cardiovascular system is the earliest developing functional organ system in the yolk sac and embryos of all vertebrate species and is necessary for gas and nutrient exchange to support life ${ }^{1-4}$. The generation of new blood vessels that comprise the cardiovascular system occurs through two similar, but distinct processes: vasculogenesis and angiogenesis ${ }^{3,5-13}$. Vasculogenesis, also known as de novo tube formation, is the process of endothelial cells (ECs) or mesodermally derived angioblasts, also referred to as endothelial precursor cells, forming new blood vessels ${ }^{1,6,11-14}$. During this process ECs become polarized and undergo lumen formation to generate both an apical and basal membrane surface, separating the lumen from the extracellular matrix environment and thus establishing a tubular structure that allows for gas and nutrient exchange across the cell $^{11,12,15,16}$. Concurrently, ECs undergoing vasculogenesis also sprout and branch to assemble into a multicellular, continuous tube network known as the capillary plexus ${ }^{1,17}$, 18 . The capillary plexus is then remodeled through the process of angiogenesis, in which new blood vessels are generated through sprouting of ECs from the capillary plexus or other established vascular networks ${ }^{5,6,8-10}$. Maturation of these vascular networks then occurs through recruitment of mural support cells (i.e. pericytes and smooth muscle cells) that help to transition vascular networks from morphogenesis to stabilization through structural support as well as establishing basal polarity by vascular basement membrane 
matrix assembly via EC and pericyte interaction ${ }^{7,19-24}$. Recently, substantial interest and effort has been made in understanding the molecular mechanisms controlling vasculogenesis, angiogenesis, and the signaling events controlling EC and mural cell interactions with considerable attention being paid to understanding the mechanisms controlling EC lumen formation. Together, these processes are critical in embryonic development ${ }^{2-4,25}$, the female reproductive cycle ${ }^{26,27}$, injury and wound repair ${ }^{28,29}$, cancer metastases and tumor growth ${ }^{30,31}$, and a number of disease states associated with ischemia, atherosclerosis, diabetes and genetic mutation as cardiovascular disease continues to be a leading cause of death globally ${ }^{32-35}$.

\section{Regulation of asymmetric cell polarity during lumen formation}

Cell polarity must be coordinated for individual cells to form into a tissue and involves the asymmetric organization of the cell surface, intracellular organelles and the cytoskeleton $^{36}$. Actin filaments and microtubules are polar polymers that comprise the cytoskeleton and are composed of globular actin and $\alpha$ - and $\beta$ - tubulin heterodimeric subunits respectively. Cytoskeletal polarity results from the unidirectional assembly of these subunits, and these polymers are highly dynamic as each end can either polymerize or depolymerize. This unicellular polarity is critical for motor proteins that associate with the cytoskeleton and function to traffic vesicles and membrane cargo along oriented actin or microtubule arrays. These motor proteins consist of myosins that associate with actin and move towards actin barbed ends, kinesins that move towards microtubule plus ends and dyneins that moves towards microtubule minus ends in the presence of the dynactin 
complex, which are often anchored at the centrosome or other microtubule minusorganizing centers ${ }^{37}$.

For actin and microtubules to assemble to into organized arrays, nucleation must occur in order to form small oligomers that can rapidly elongate ${ }^{38,39}$. The assembly of polarized actin arrays is regulated in part by actin nucleation factors such as the actinrelated protein-2/3 (Arp2/3) complex and formin-family proteins activated upstream by Rho-family GTPases ${ }^{40,41}$, whereas a mechanism of organization of microtubules results from cortical capture of microtubule plus ends regulated by proteins associated with microtubule plus ends and cortical factors controlled by Rho GTPases ${ }^{42,43}$. Furthermore, maintenance of polarity and microtubule stability has been associated with differences in post-translationally modified tubulins, such as detyrosination, acetylation and polyglutamylation as these modifications have been associated with enhanced kinesin-1 binding to microtubules to facilitate transport processes in neurons and fibroblasts ${ }^{44-47}$. Another component of cellular asymmetry results from centrosome orientation and selective stabilization of a subset of microtubules, which serves to position the centrosome around the nucleus. Control of this orientation has been associated with the activity of Cdc42, partitioning defective protein (PAR)6, atypical protein kinase C (aPKC) and dynein ${ }^{48-50}$. However, Cdc42 effectors including IQ motif- containing GTPase-activating protein-1 (IQGAP1), myotonic dystrophy kinase-related Cdc42binding kinase (MRCK) and mammalian formin diaphanous-1 (mDia1) have also been implicated in controlling centrosome orientation suggesting that the mechanism of orientation may depend on cell type or distinct downstream pathways ${ }^{51-53}$. 
The asymmetric distribution of cytoskeletal components and its ability to regulate components of polarity signaling is crucial in both endothelial and epithelial de novo lumen formation in the mechanism of basal to apical membrane transport ${ }^{11,12,15,36,37,54}$. It has been demonstrated in models of epithelial and endothelial lumen formation that activity of the Cdc42/Par3/Par6/aPKC complex is required ${ }^{55-57}$. Interestingly, Par3/Par6 has been reported to associate with VE-Cadherin, which has a key role in the development of the vasculature and serves as the principal EC adherens junction molecule and was also shown to be a regulator of EC polarity ${ }^{58-60}$. It was recently reported that acetylated tubulin and detyrosinated tubulins localize at the subapical domain of the developing EC lumen and that these tubulin modifications correlate with EC lumen formation whereas F-actin is distributed basally. Furthermore, these tubulin modifications are controlled by key microtubule tip complex proteins, and the tubulin deacetylases HDAC6 and SIRT2 negatively regulate EC tubulogenesis ${ }^{15}$. Additionally, a critical component of endothelial basal polarity is the recruitment of mural cells to the abluminal EC surface and the deposition of vascular basement membrane matrix ${ }^{21,22}$. Thus, multiple regulators control asymmetric polarization necessary for de novo EC lumen formation and maintenance of a functional vasculature although many of the molecular mechanisms associated with EC polarity are not well understood including those controlled by flow.

\section{A comparison of endothelial and epithelial de novo lumen formation}

The mechanism of de novo lumen formation is a critical step that precedes tubulogenesis in both the development of the blood filled vasculature consisting of EC 
tubular networks, as well as critical tubular structures that characterize epithelial tissues such as the kidney, lung and brain. Recently, great advancements in the understanding of the molecular mechanisms contributing to lumen formation and tubulogenesis in both ECs and epithelial cells have been made from the development of both in vitro models utilizing culture of endothelial and epithelial cells in three-dimensional extracellular matrix environments and in vivo models investigating the development of the vasculature and epithelial tubular structures in D. melanogaster, C. elegans, mice, and zebrafish ${ }^{11-13 \text {, }}$ ${ }^{61-63}$. From these models, it has become evident that lumen formation consists of a series of steps in which cells first become polarized to establish where membrane material will be trafficked to develop the lumen area, then growth of the apical membrane surface and expansion of the lumen, and finally maturation of the lumen area to the appropriate diameter and stabilization of the structure to begin to fulfill its physiological roles. These models have identified that lumen initiation, expansion and maturation consists of a number of highly coordinated cell signaling events. However, the intricate details of these cell-signaling events remain to be well understood and are of great interest.

During epithelial lumen formation, work in Madin-Darby Canine Kidney (MDCK) 3D cyst models demonstrated that the initial cue for apicobasal polarization results from $\beta 1$ integrin interaction with different protein components of the extracellular matrix including laminins, collagens, entactin and perlecan and downstream signaling through the GTPase Rac $1^{64-66}$. As these cells are interacting with one another in multicellular cysts, intercellular junctions form, consisting of polarized Epithelial (E)cadherins that induce the differential distribution of phosphoinositide species to specify membrane identity where $\operatorname{PtdIns}(3,4,5) P_{3}$ localizes to the basolateral membrane, while 
$\operatorname{PtdIns}(4,5) P_{2}$ functions in part to define apical identity ${ }^{55,67}$. Additionally, polarity is controlled by key Par and Crumbs protein complexes that regulate apical polarity while the Scribble protein complex is a regulator of basal polarity. These different polarity regulators then have a critical role in the polarized trafficking of membrane vesicles from the cell periphery to the apical membrane initiation site, which proceeds to form the preapical patch as vesicles fuse with one another before lumen expansion in a process referred to as cell hollowing. In this mechanism, PTEN is recruited to the apical membrane by the Cdc42/Par3/Par6/aPKC signaling complex to maintain apical PtdIns $(4,5) P_{2}$ in association with Annexin $2^{55}$. Rab11A and Rab8A positive vesicles were then shown to associate with these complexes and control delivery of vesicles to the apical membrane initiation site along with exocyst regulators as discussed in additional detail below ${ }^{66,68}$.

In contrast, the identification of polarity regulators controlling de novo lumen formation of ECs has proven a significant challenge, as apicobasal markers have been difficult to identify. However, previous work has identified a number of GTPase regulators controlling EC polarity, as well as the contributions of asymmetric distribution of the cytoskeleton and recruitment of mural cells to the abluminal surface as discussed above $^{15,21,22,54,69-71}$. An important distinction between epithelial cells and ECs is that epithelial tubes are specialized for apical secretion or absorption and are thus dependent on specialized organization of transmembrane proteins and cytoskeletal components to accomplish this task whereas EC-lined tubes are primarily channels for blood flow and gas-exchange. Thus, EC requirement for specialized polarity appears to be less compared to the epithelium whereas flow forces in contact with the apical membrane surface likely 
has a major role in EC polarity signaling ${ }^{12}$. Additionally, EC tubulogenesis in vitro (1-3 days) is observed to be much more rapid than that of epithelial tubulogenesis (1-2 weeks $)^{10,12,72,73}$. In accordance with this observation of ECs rapidly generating lumens during tubulogenesis, it has been reported that ECs are able to undergo pinocytic intracellular vacuolization to rapidly generate and transport vacuoles from the basal membrane to the site of apical lumen formation where they coalesce ${ }^{74-80}$. Great effort has been put forth in investigating the molecular mechanisms controlling pinocytic vacuolization and other signaling events during EC tubulogenesis and details of significant findings are discussed below.

\section{The endothelial lumen signaling cascade}

\section{An overview of key signaling mechanisms}

Considering EC lumen morphogenesis and tubulogenesis, a large body of research suggests that key cell signaling events include initiation through integrins, tyrosine kinase receptors, G-protein coupled receptors and matrix metalloproteinases ${ }^{7,57}$, $70,73-75,77,81-88$. These molecules then activate a downstream signaling cascade involving kinase activity of protein kinase $\mathrm{C}(\mathrm{PKC})$ isoforms and Src family kinase (SFK) members, activation of small GTPases that regulate cytoskeletal rearrangements through association with downstream GTPase effectors and activation of the Raf-MEK-Erk pathway $^{56,57,73,76,86,89}$. These signaling events initiate development of the lumen and capillary assembly through membrane trafficking events and digestion of the extracellular matrix environment to expand the lumen area as well as allowing for migration of ECs in matrix tunnel spaces to assemble into a network of EC tubes by matrix metalloproteinase 
activity. Additionally, these signaling events are regulated by key microtubule tip

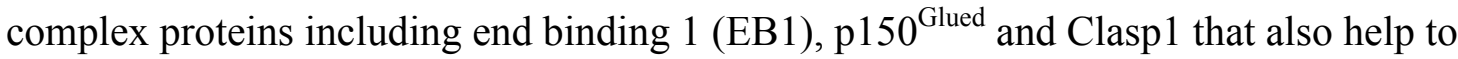
regulate asymmetric EC cytoskeletal polarity through tubulin acetylation and detyrosination ${ }^{15}$.

\section{Integrin and ECM interaction during EC tubulogenesis}

The extracellular matrix is composed of different families of molecules that include glycosaminoglycans and proteoglycans, collagens and non-collagenous glycoproteins ${ }^{90}$ and among the vasculature, these matrix proteins can vary depending on the state the vessel is in, e.g., quiescent, injured or angiogenic ${ }^{85,91}$. For ECs, the extracellular matrix not only serves as a scaffold that is necessary for EC migration, survival and proliferation but also as a critical morphogenic cue during vasculogenesis and angiogenesis through integrin signaling with extracellular matrix components such as the collagens and fibrin/fibronectin ${ }^{28,74,75,77,82,85,87,88,91,92}$. The integrins are a major cell surface receptor class composed of $\alpha$ - and $\beta$ - chains that heterodimerize into different combinations dependent on ligand interaction with a particular matrix protein. In mammalian genomes, 18 different $\alpha$ - chains and 8 different $\beta$ - chains have been identified to assemble into 24 different combinations ${ }^{93,94}$. Collagens are of the most abundant proteins in the animal kingdom and in collagen type I matrix environments, the collagen integrin receptors $\alpha 1 \beta 1$ and $\alpha 2 \beta 1$ have been identified to be necessary in initiating EC tubular morphogenic events ${ }^{74,90,95}$. Furthermore, in fibrin and fibronectin enriched matrices that are typically induced in response to tissue injury, $\alpha 5 \beta 1$ integrin 
was primarily identified to be necessary for EC tubular morphogenesis, whereas a possible role for $\alpha v \beta 3$ integrin was identified ${ }^{85,88}$.

\section{The role of hematopoietic cytokines, FGF-2 and VEGF signaling in EC tubulogenesis}

Vascular endothelial growth factor (VEGF) has previously been reported to control EC tubulogenesis and has been a primary area of focus for the vascular biology field in evaluation of its signaling mechanisms downstream of receptor-ligand binding ${ }^{96-}$ ${ }^{99}$. However, the dependence of other critical factors that act in concert with VEGF during EC tubulogenesis has been difficult to assess due to complexities associated with the experimental models used. Interestingly, using defined serum-free culture conditions it was demonstrated that VEGF and fibroblast growth factor-2 (FGF-2) addition alone or in combination were not sufficient to stimulate EC tubulogenesis in $3 \mathrm{D}$ collagen matrices. However, the addition of hematopoietic cytokines stem cell factor (SCF), interleukin-3 (IL-3) and stromal derived factor-1 $\alpha$ with FGF-2 (as FGF-2 is fundamental for EC survival, arteriogenesis and branching morphogenesis) ${ }^{98,100,101}$ allowed for EC tubulogenesis and capillary assembly in 3D collagen matrices. Additionally, it was shown that VEGF and FGF-2 function as critical priming cues that stimulate EC morphogenesis in response to the hematopoietic cytokines suggesting that VEGF signaling and hematopoietic cytokine signaling are controlling distinct pathways regulating EC tubulogenesis.

A number of studies have documented that ECs can produce SCF and that they

express its receptor, the tyrosine kinase $\mathrm{c}-\mathrm{Kit}^{102-104}$. Upon ligand binding, c-Kit dimerizes 
and induces autophosphorylation of tyrosine residues in the cytoplasmic tail resulting in the recruitment of a number of signaling molecules controlling various downstream pathways. Interestingly, in addition to activation of JAK-STAT pathways, activation of SFKs and the Ras-Raf-MEK-Erk pathway are induced in response to c-Kit signaling ${ }^{105}$, and both of these signaling pathways are critical regulators of EC tubulogenesis ${ }^{56,89}$. ECs have also been reported to express IL-3 receptor ${ }^{106}$. IL-3 binding to low affinity IL-3R $\alpha$ and subsequent heterodimerization with the common $\beta$ subunit forms a high-affinity receptor that signals through the JAK-STAT pathway, primarily through JAK2 and STAT5. However, IL-3 signal transduction is also capable of activating the Ras-RafMEK-Erk kinase cascade ${ }^{107}$. SDF-1 $\alpha$, a member of the C-X-C cytokines, in turn signals through the G-protein coupled receptor $\mathrm{CXC} 4$ and activates downstream signaling pathways including PI-3 kinase, Rho family GTPases, and the Ras-Raf-MEK-Erk kinase cascade $^{108}$.

Together, the transduction of signals from these pathways along with FGF-FGFR signaling ${ }^{101}$ suggests that synergistic activation of the Ras-Raf-MEK-Erk kinase cascade is critical during EC tubulogenesis. It was also demonstrated that mRNA and protein expression of c-Kit, IL-3R $\alpha$ and CXCR4 were increased in response to priming with VEGF and FGF-2, which correlates with a stimulated EC morphogenic response during tubulogenesis ${ }^{73}$ supporting this notion. Because GTPase signaling also activates this pathway in concert with Src, which is activated in response to SCF signaling, it is likely that these cytokines influence activation of key downstream GTPases involved in EC tubulogenesis as well. However, details of this possible molecular mechanism have not yet been elucidated. 


\section{MT1-MMP proteolytic activity is required for the formation of vascular guidance tunnels and activation of $\mathrm{Cdc42}$ during EC tubulogenesis}

The matrix metalloproteinases (MMPs) are a family of enzymes that regulate a number of cell processes including ECM degradation, proteolysis of cell surface proteins,

liberation of growth factors and tissue morphogenesis, including vascularization ${ }^{86,109-111}$. During EC tubulogenesis, it was shown that MT1-MMP activity is necessary for the generation of vascular guidance tunnels within 3D collagen matrices that are necessary for EC lumen formation ${ }^{86}$. Furthermore, these vascular guidance tunnels create space within the ECM that allow for the migration of ECs along these surfaces in a MMPindependent manner that allows for vascular remodeling, and also allows for migration of pericytes that are recruited to the EC abluminal surface to stabilize and mature capillary networks ${ }^{21,86}$. Importantly, MT1-MMP proteolytic activity and subsequent formation of vascular guidance tunnels is coordinated with other molecules controlling EC tubulogenesis as inhibition of $\alpha 2$ integrin, PKC $\varepsilon$ and Src blocked tunnel formation ${ }^{86}$. The importance of MT1-MMP proteolytic activity during vascular morphogenesis has also been demonstrated in vivo where MT1-MMP deficient mice were found to have severe defects in angiogenic responses supporting the critical importance of MT1-MMP in vascular development and remodeling ${ }^{112}$. Critical to MT1-MMP's function in controlling EC tubulogenesis is its association with the GTPase Cdc42. It was shown that Cdc42 and MT1-MMP associate in a multiprotein signaling complex with other key regulators of EC tubulogenesis and their activity is interdependent with one another in the context of EC tubulogenesis in 3D ECM environments. This interdependent relationship was shown by 
blockade of vascular guidance tunnel formation with siRNA suppression of Cdc42 and in contrast, reduced activation of Cdc42 by inhibition of MT1-MMP during lumen formation in 3D collagen matrices. However, migration of ECs and activation of Cdc42 on a 2D surface was not affected by inhibition of MT1-MMP activity ${ }^{57}$. Because of MT1MMP's necessity during EC tubulogenesis and vascular morphogenesis, it is likely that MT1-MMP activity influences and controls other key signaling mechanisms downstream.

\section{Src family kinase and protein kinase $\mathrm{C}$ activity in controlling $\mathrm{EC}$ tubulogenesis}

Targets of signal transduction downstream of the integrins and key receptors include the SFKs ${ }^{113-115}$ comprised of nine members including Src, Fyn, Yes, Lck, Hck, Blk, Fgr, Lyn and Yrk ${ }^{116}$. The SFKs are a family of non-receptor protein tyrosine kinases that regulate a number of signaling transduction pathways downstream of integrins, receptor protein tyrosine kinases, G Protein-coupled receptors, cytokine receptors and others. SFKs become active in these signal transduction pathways by phosphorylation of the Y416 site and dephosphorylation of the inhibitory Y 527 site $^{116}$. In human endothelial cells, the more prominently expressed SFKs include Src, Yes, Fyn, and Lyn ${ }^{89}$ and it was previously demonstrated that the tyrosine phosphorylation activity of Src is critical during EC tubular morphogenesis in vitro ${ }^{89,117}$ and in vivo ${ }^{118,119}$.

In addition to activation by integrins during EC tubular morphogenesis, the PKC isoform PKC $\varepsilon$ was demonstrated to be an activator of Src signaling and subsequent downstream tyrosine phosphorylation events of ECs suspended in 3D collagen matrices ${ }^{89}$. The PKC isoforms comprise a family of serine/threonine kinases that are divided into three different subfamilies based on differences in $\mathrm{NH}_{2}$-terminal regulatory 
domain structure. The conventional isoforms, $\mathrm{PKC} \alpha, \mathrm{PKC} \beta \mathrm{I}$ and the alternatively spliced PKC $\beta I I$ and $\mathrm{PKC} \gamma$ contain a regulatory domain termed $\mathrm{C} 1$ that functions as a diacylglycerol (DAG)-/phorbol 12-myristate 13-acetate (PMA and also known as TPA)binding motif. Additionally, these isoforms also contain a regulatory domain termed $\mathrm{C} 2$ that binds anionic phospholipids in a process that is calcium-dependent. The novel PKC isoforms, $\mathrm{PKC} \delta, \mathrm{PKC} \theta, \mathrm{PKC} \varepsilon$ and $\mathrm{PKC} \eta$, also contain a similar $\mathrm{C} 1$ domain that functions as a DAG-/TPA-binding motif and a similar C2 domain. However, the C2 domain of novel PKC isoforms lack the critical calcium coordinating acidic residues and thus novel PKCs are primarily activated by agonists that promote DAG accumulation or TPA with no calcium requirement. The atypical $\mathrm{PKC}$ isoforms, $\mathrm{PKC} \zeta$ and $\mathrm{PKC} / \lambda$, lack a $\mathrm{C} 2$ domain and possess an atypical $\mathrm{C} 1$ domain that binds phosphatidylinositol $(3,4,5)-$ triphosphate $\left(\mathrm{PIP}_{3}\right)$ and ceramide. Their regulatory region also contains a unique domain from the other PKC subfamilies known as the Phox and Bem 1 (PB1) that mediates interactions with other proteins containing PB1 ${ }^{120}$.

A number of previous studies have implicated that TPA strongly stimulates EC morphogenic events both in vitro ${ }^{74,121-123}$ and in vivo ${ }^{124}$. A combination of chemical inhibitor and siRNA mediated RNA interference experiments then identified critical roles for novel PKC $\varepsilon$ and atypical $\mathrm{PKC} \zeta$ during EC tubulogenesis in $3 \mathrm{D}$ collagen matrices ${ }^{56}$. During EC tubulogenesis, $\mathrm{PKC} \varepsilon$ kinase activity was shown to be necessary for activation of Src kinase signaling, which activates the Raf-Mek-Erk1/2 kinase cascade through activation of the $\mathrm{p} 21$ activated kinases (PAKs) PAK2 and PAK4 ${ }^{89}$. In addition to integrin, SFK and PKC regulation of EC tubulogenesis, a continually growing amount of evidence has implicated a critical role for regulation of this morphogenic process by members of 
the Ras superfamily of GTPases and several studies have identified key relationships between GTPase activity and that of integrin, SFK, and PKC signaling during EC tubulogenesis $^{56,57,89}$.

\section{The role of Ras superfamily GTPase regulation of endothelial lumen formation and vessel stabilization \\ An overview of Ras superfamily GTPases}

The Ras superfamily of GTPases is comprised of over 150 members that are separated into five families based on sequence and function similarity: Ras, Rho, Rab and Ran, Arf (Figure 1.1). These proteins function as monomeric G proteins that share a similarity to the heterotrimeric $\mathrm{G}$ protein $\alpha$ subunits and possess a common biochemical mechanism in acting as GDP/GTP-regulated molecular switches. A number of these GTPases also contain post-translational lipid modifications that serve to direct these proteins to various subcellular locations to execute their functions. The majority of these GTPases possess an intrinsic ability of GTP hydrolysis to GDP and cycling between GTP- or GDP-bound states is regulated by two additional classes of proteins, guanine nucleotide exchange factors (GEFs) and GTPase activating proteins (GAPs) ${ }^{125,126}$. An additional form of regulation for the Rab and Rho family of GTPases comes from association with guanine nucleotide dissociation inhibitors (GDIs), which function to sequester these GTPases in the cytosol ${ }^{127,128}$. GTPases are capable of associating with a number of different downstream effector molecules to regulate a variety of cellular processes such as proliferation, cell polarity, migration, membrane trafficking and 
morphogenesis ${ }^{125,126}$ and in the Rho family for example, identification of GTPase effectors has been a significant challenge since a majority of the effectors do not have a conserved domain ${ }^{128,129}$. In addition to association with a variety of effector molecules, individual GTPases are also regulated by several GEFs and GAPs that may have more than one GTPase target and thus demonstrate the complexity of GTPase signaling in regulation of cellular processes ${ }^{130}$.

\section{Rho-family GTPase regulation of cytoskeletal organization, cell polarity and lumen formation}

Among the most well studied GTPases are the Rho family members Cdc42, Rac1, and RhoA. Studies in the early 1990s identified these particular GTPases to be regulators of the actin cytoskeleton ${ }^{131-134}$ and global deletion of Cdc42 and Rac1 in vivo resulted in lethality at early stages demonstrating a critical function for these GTPases during early development ${ }^{135,136}$. In response to different extracellular cues, RhoA was shown to promote the formation of actin stress fibres ${ }^{131}$, while Rac1 activation promoted the formation of lamellipodia/membrane ruffles ${ }^{132}$. Shortly thereafter, other studies demonstrated that upon activation Cdc42 induced actin-rich microspikes, known as filipodia, at the cell periphery and also activated Rac activity via lamellipodia formation ${ }^{133,134}$. Through great effort, work in the mid-1990s identified between 20 and 30 potential downstream targets that interact with $\mathrm{Cdc} 42$, Rac1, and RhoA in a GTPdependent manner and revealed that these GTPases were capable of regulating several cellular signaling pathways ${ }^{129}$. One of the earliest identified downstream effectors for Cdc42 and Rac1 were PAKs ${ }^{137}$ and shortly thereafter Rho-associated kinase (ROCK) was 
identified as a downstream effector of RhoA that activates myosin II by phosphorylation and inactivation of myosin light chain phosphatase ${ }^{138,139}$. Cdc42 was also shown to be a key regulator of cell polarity and establishing asymmetry during epithelial morphogenesis, asymmetric cell division and directed cell migration events through its association with a Par6-PKC $\zeta$ complex ${ }^{55,140-144}$. Because of the critical role for these GTPases in regulation of actin reorganization and cell polarity, there has been great interest in understanding how these molecules may regulate tube morphogenesis events and cytoskeletal reorganization in both endothelial and epithelial tissues.

A necessary requirement for $\mathrm{Cdc} 42$ and Rac 1 activity during tubulogenic morphogenesis events was first identified in vitro utilizing ECs suspended in 3D collagen or fibrin matrices under stimulation of TPA whereas a role for RhoA during these events was not supported ${ }^{76,77}$. Additional work utilizing ECs undergoing tubulogenesis in 3D collagen matrix environments has demonstrated that $\mathrm{Cdc} 42$ associates with PAK2, PAK4, Par3, Par6B ${ }^{56,57}$, members of the junction adhesion molecule (Jam) family JamB and JamC, $\alpha 2$ integrin, MT1-MMP ${ }^{57}$ and $\mathrm{Src}^{89}$ in signaling complexes that function to activate the Raf-MEK-Erk1/2 kinase cascade, establish apical and basal polarity and promote lumen morphogenesis in conjunction with PKC activity ${ }^{56,57,89}$ (Figure 1.2). Recent evidence in vivo has also demonstrated a critical role for Cdc42 in the initiation of lumen formation as well as maintenance of tube structures by utilizing an inducible endothelial specific knockout model where EC adhesion, polarity and vascular remodeling ability are impaired, implicating that Cdc 42 activity is critical in both vascular development and maintenance ${ }^{145}$. Similarly, endothelial specific deletion of Rac1 results in vascular development defects ${ }^{146}$. Work in vitro and in vivo has also shown 
that $\mathrm{Cdc} 42$ activity and association with Par6/atypical PKC is required for lumen formation and trafficking of vesicles to the apical membrane during epithelial tube morphogenesis in MDCK cyst models in differing ECM environments ${ }^{55,66,147,148}$ and in the developing pancreas ${ }^{149}$. Rac1 function has also been characterized in epithelial tube morphogenesis events where expression of DN Rac1 inversed polarity, inhibited lumen formation and resulted in misassembled laminin at the basement membrane ${ }^{64}$ and Rac1 functions downstream of $\beta 1$ integrin signaling during this process to properly orient epithelial cell polarity ${ }^{65,150}$. Additionally Rac1, and not $\mathrm{Cdc} 42$, is needed for epithelial tight junction formation ${ }^{151}$. Therefore, in both endothelial and epithelial tissues, Cdc42 and Rac1 possess similar functional requirements in promoting lumen morphogenesis although details of the molecular signaling events regulated by these two GTPases are still the focus of ongoing investigation.

Conversely, a number of studies suggest that activation of RhoA signaling impairs tubulogenesis events in both endothelial and epithelial tissues. Evidence from in vitro studies of endothelial lumen formation demonstrated that delivery of a constitutively active (CA) form of RhoA by recombinant adenovirus infection inhibited tubulogenesis and induced EC death ${ }^{76}$. It was also shown that inhibition of EC lumen formation through the use of microtubule disrupting agents, e.g. vinblastine, colchicine and nocodazole, is dependent on activation of RhoA signaling where pretreatment of ECs with C3 exoenzyme or recombinant adenovirus delivery of dominant negative (DN) RhoA completely blocked induced tube collapse ${ }^{152}$. Furthermore, Erk-MAPK kinase activity and Src kinase activity were identified to suppress Rho-ROCK signaling during EC morphogenesis events in vitro and in vivo ${ }^{117,153}$. In addition to regulation by these kinase 
signaling pathways, upstream regulation of RhoA activity during EC tubulogenesis is controlled by the GTPase effector Rasip1, which is highly expressed in ECs, and its association with the RhoGAP Arhgap29. Rasip1 null mice exhibit vascular defects in early development where ECs assemble into cord-like structures where the lumen fails to develop. In vitro studies of Rasip1 or Arhgap29 knockdown during vasculogenesis in 3D collagen matrices recapitulated these results and demonstrated increased detection of endogenous active RhoA-ROCK signaling by detection of downstream phosphorylated myosin phosphatase targeting subunit (pMYPT) as well as increased detection of phosphorylated myosin light chain (pMLC) ${ }^{154}$. In further support of suppression of RhoROCK activity during lumen morphogenesis events, studies in epithelial models of lumen formation have shown that a $\beta 1$ integrin/focal adhesion kinase (FAK)/p190A RhoGAP complex functions to positively regulate epithelial lumen formation by repressing RhoA signaling ${ }^{155}$. Additionally, chemical inhibition of ROCK by the drug Y-27632 is able to rescue polarity and lumen defects induced by use of anti- $\beta 1$ integrin blocking antibody or

DN Rac1 expression in MDCK cyst models ${ }^{150}$. Therefore, it is evident that during early lumen morphogenesis events, a critical balance must be maintained in order to activate Cdc42 and Rac driven signaling and inhibit RhoA signaling.

\section{Ras superfamily members implicated in endothelial morphogenesis and vessel integrity}

Although considerable attention has been focused on the investigation of the role of the classic Rho-family GTPases during early EC tubulogenesis and vessel maturation, a number of studies have focused on the role of additional Ras superfamily members. A 
recent study showed that a target of the transcription factor ERG, which is a member of the ETS family of transcription factors highly enriched in endothelial cells, is the GTPase RhoJ. RhoJ activity was shown to be necessary during early vasculogenesis events and to regulate phosphorylated activation of critical downstream kinases in the lumen-signaling cascade such as PAK2, PAK4, B-Raf, C-Raf, Src and ERK through association in multiprotein signaling complexes with $\mathrm{Cdc} 42^{156}$. Of additional interest is the role of Rap1 isoforms, Rap1A and Rap1B, during endothelial morphogenic events ${ }^{157}$. Global deletion of Rap1A or Rap1B in mouse models have resulted in partial embryonic lethality phenotypes characterized by bleeding and edema and impaired angiogenesis ${ }^{158-162}$, while combined deletion of Rap1A and Rap1B resulted in embryonic lethality between E8.5E10.5 due to major malformation ${ }^{157}$. However, endothelial-lineage restricted deletions using a Tie2-Cre driven model demonstrated impaired angiogenesis phenotypes among an otherwise normal lifespan when both alleles of Rap1B were deleted ${ }^{162}$. In contrast, Tie2-Cre deletion of both alleles in Rap1A and Rap1B resulted in embryonic lethality due to hemorrhage between E10.5-E13.5 showing that at least one Rap1 isoform is required for normal vascular development and that there may be redundancy in function between the two isoforms ${ }^{157}$. Morpholino mediated inhibition of Rap1B expression during zebrafish development also lead to vascular malformations, recapitulating roles for Rap1B function during mouse development ${ }^{157,162,163}$.

Work from cellular models investigating the role of Rap1 in the endothelium has identified a primary functional role for Rap1 in the stabilization of cell-cell junctions. Evidence for the molecular mechanism of this function has shown that Rap1 associates with Afadin and regulates adherens junction formation. Deletion of Afadin in the 
endothelium utilizing a Tie2-Cre driven system showed a markedly impaired angiogenesis phenotype in the retina and the postnatal viability of the homozygous knockout mice in this model is greatly reduced, implicating the role of Rap1-Afadin activity in other aspects of vasculogenesis ${ }^{164}$.

Another Rap1 effector identified to be a critical regulator of vascular morphogenesis and integrity is KRIT1, or CCM1 $1^{79}, 165$. Cerebral cavernous malformations (CCMs) are vascular defects of the central nervous system observed as brain angiomas that are susceptible to hemorrhaging and can lead to seizure or stroke ${ }^{166}$. In addition to mutations in CCM1/KRIT1 $1^{167,} 168$, two other loci have been implicated in autosomal forms of CCM including CCM2/OSM/malcavernin ${ }^{169}$ (where evidence has shown Rac1 as a binding partner) ${ }^{170}$ and CCM3/PDCD10 $0^{171}$. Importantly, it was shown that endothelial Rap1 activity is necessary for KRIT1's interaction with junctional molecules ${ }^{172}$ where KRIT1 binds Heart-of-glass (HEG1) receptor ${ }^{173}$ and associates with junctional molecules such as $\beta$-catenin and $\operatorname{Afadin}^{174}$. Recent studies have also shown evidence that active Rap1 associates with Rasip1 and its binding partner Arhgap29 to negatively regulate RhoA activation and positively regulate endothelial barrier activity implicating a role for this signaling cascade in stabilization of the endothelium ${ }^{175-177}$. Furthermore, CCM2 was shown to be required for EC tubulogenesis in vitro and homozygous CCM2 mutated mice become lethal during mid-gestation due to defects in angiogenesis and vascular patterning. Importantly, loss of CCM2 was shown to induce formation of actin stress fibers and activate RhoA ${ }^{178}$ and this supports the conclusion that regulation of GTPase activity is critical in different vascular morphogenesis events. 
While several GTPases have been identified to have essential functions for development and maintenance of the endothelium, the role for many other Ras superfamily GTPases members has not been elucidated. A significant amount of work has identified critical molecular mechanisms of GTPase regulation of early vasculogenesis events, yet this has been primarily limited to the Rho family members Cdc42, Rac1 and RhoA and further investigation is required to understand the contribution of other novel GTPase regulation of EC tubulogenesis and vascular maturation. Furthermore, the molecular mechanisms controlling the regulation of GTPase signaling through GEF or GAP activity are not well understood and continued work is required to identify and understand the role of key GTPase regulators during tubulogenesis and the mechanisms controlling regulation of their activity.

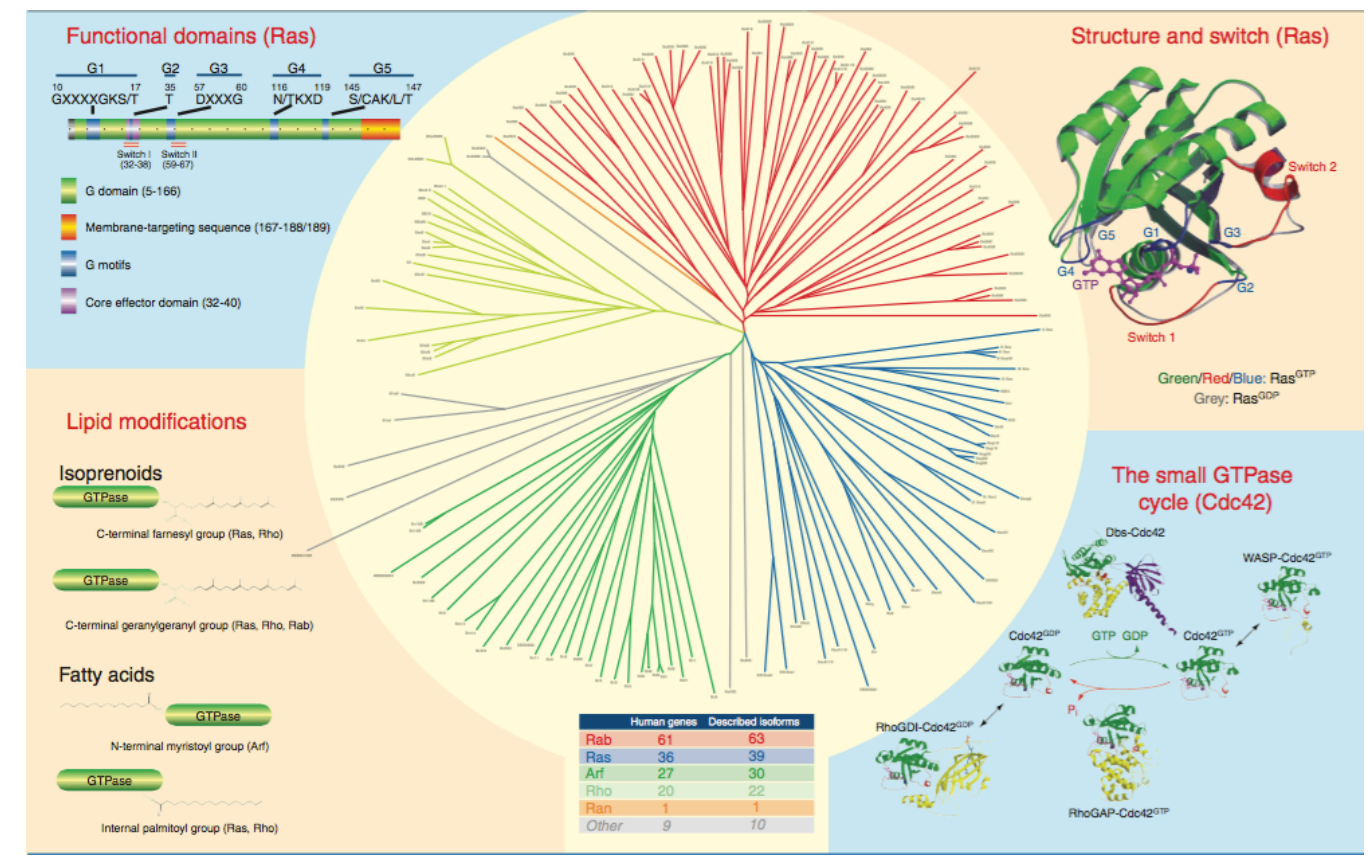

Figure 1.1. The Ras superfamily of small GTPases. Reproduced with permission. Journal of Cell Science ${ }^{126}$. http://jcs.biologists.org/content/118/5/843 


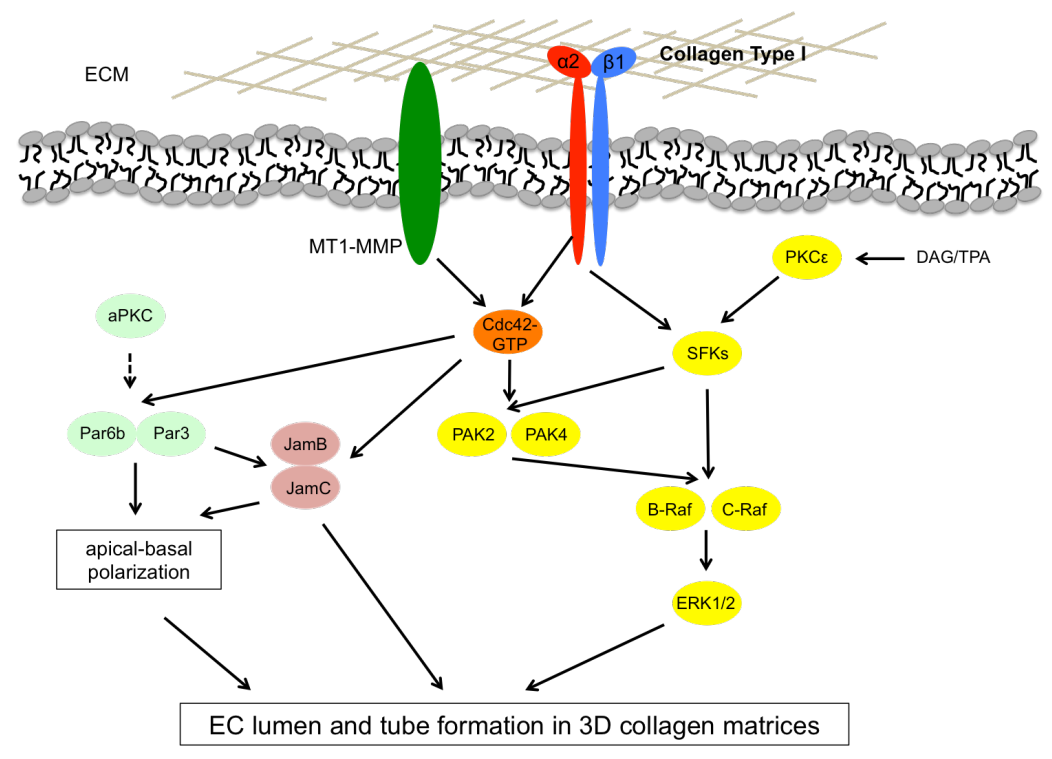

Figure 1.2. Cdc42-associated molecular signaling complexes control EC lumen formation and tubulogenesis in $3 D$ collagen matrices.

\section{Regulation of membrane trafficking events in lumen formation}

Advancements in imaging techniques have identified that ECs suspended in 3D matrices are able to undergo rapid intracellular vacuolization and coalescence of these vacuole structures to form the lumen during tubulogenesis ${ }^{74-78,179}$. Of importance is that these structures have been observed in vivo as well ${ }^{12,21,78-80,87,180,181}$. Although this is not the only observed mechanism of EC lumen formation in vitro and in vivo ${ }^{11-13,61,182}$, this mechanism represents a way in which individual ECs are able to quickly generate lumens and assemble into multicellular tube structures to support the needs of embryonic development ${ }^{12}$. What has been observed and described in vitro is a process similar to macropinocytosis in which large areas of basal membrane are pinocytosed and trafficked to the nascent apical membrane position where coalescence of the pinocytic vacuoles 
occurs as the lumen develops and expands ${ }^{74,75,179}$. There is also evidence for fusion of other intracellular vesicles with pinocytic vacuoles during EC lumen formation as Weibel-Palade bodies (WPBs) secretory granules were observed to fuse and transfer their contents (e.g. von Willebrand factor) into early vacuole and lumen structures ${ }^{74,77}$. While there is evidence for observation of these structures during lumen formation, the mechanism of how these vacuoles are generated, trafficked in a polarized manner and fuse with one another to form the lumen is not well understood. Furthermore, little is known about the contribution of other membrane vesicles, such as endosomes, exosomes and caveolae to endothelial lumen formation and continued work is required to elucidate their role.

Endocytosis describes the de novo production of intracellular membrane compartments from the plasma membrane phospholipid bilayer whereas exocytosis describes the opposite process of transportation of internal membrane compartments to the plasma membrane surface. A number of different endocytic mechanisms have been described in mammalian cells such as clathrin mediated ${ }^{183,184}$ and clathrin-independent endocytosis, caveolae/caveolin-1 dependent mechanisms, macropinocytosis, phagocytosis ${ }^{185-187}$ and others ${ }^{188}$. Interestingly, Rac ${ }^{189}$, PAK $^{190}$ and Src kinase activity ${ }^{191}$ have been associated with the generation of macropinsomes and their necessity during EC lumen formation may be in part related to the generation of these macropinocytic membrane compartments. ECs are also one of the cell types with the highest expression of caveolin- $1^{192}$. Knockdown of caveolin-1 in ECs using antisense oligonucleotides reduced capillary formation in vitro in 3D fibrin gel assays and in vivo in the chorioallantoic membrane system of chick embryos ${ }^{193}$, whereas increased expression of 
caveolin-1 increased capillary-like assembly in an in vitro Matrigel system ${ }^{194}$. Many proteins have been identified to associate with caveolae in endothelial cells ${ }^{195}$ including critical regulators of endothelial lumen formation and tubulogenesis such as Src, Yes ${ }^{192,}$ ${ }^{196}, \operatorname{Raf}^{197}$ and $\mathrm{MEK}^{192}$ and together these results implicate an important role for caveolae/caveolin-1 dependent trafficking mechanisms in EC function.

With the vast number and variety of membrane vesicles containing various cargo comes a requirement for a highly organized and regulated system to deliver these vesicles to specific locations in the cell. The largest family of the Ras superfamily of GTPases is the Rab GTPase family and its members primarily regulate the coordination of these membrane trafficking events ${ }^{198-202}$. In humans, there are currently more than 60 identified Rab members that are localized to distinct intracellular membrane compartments associated with the early endosome, recycling endosome, late endosome, trans-golgi network, secretory vesicles and others as shown in an epithelial cell represented in Figure $1.3^{199,201,203,204}$. Studies of epithelial morphogenesis and lumen formation have identified that Rab11a activity and downstream activation of Rab8a via its GEF Rabin8 are necessary for establishment of polarity and lumen formation ${ }^{62,68,205-208}$ and this process is dependent on association with Myosin $\mathrm{Vb}$ motor protein to properly traffic vesicles to the apical membrane initiation site ${ }^{209}$. Because of the observed Rab requirement for lumen formation in epithelial models of morphogenesis and the observations of organized membrane trafficking of pinocytic vacuoles in EC lumen formation, investigation into the role of Rab GTPases and other membrane trafficking regulators during EC lumen formation and tubulogenesis is of great importance. 


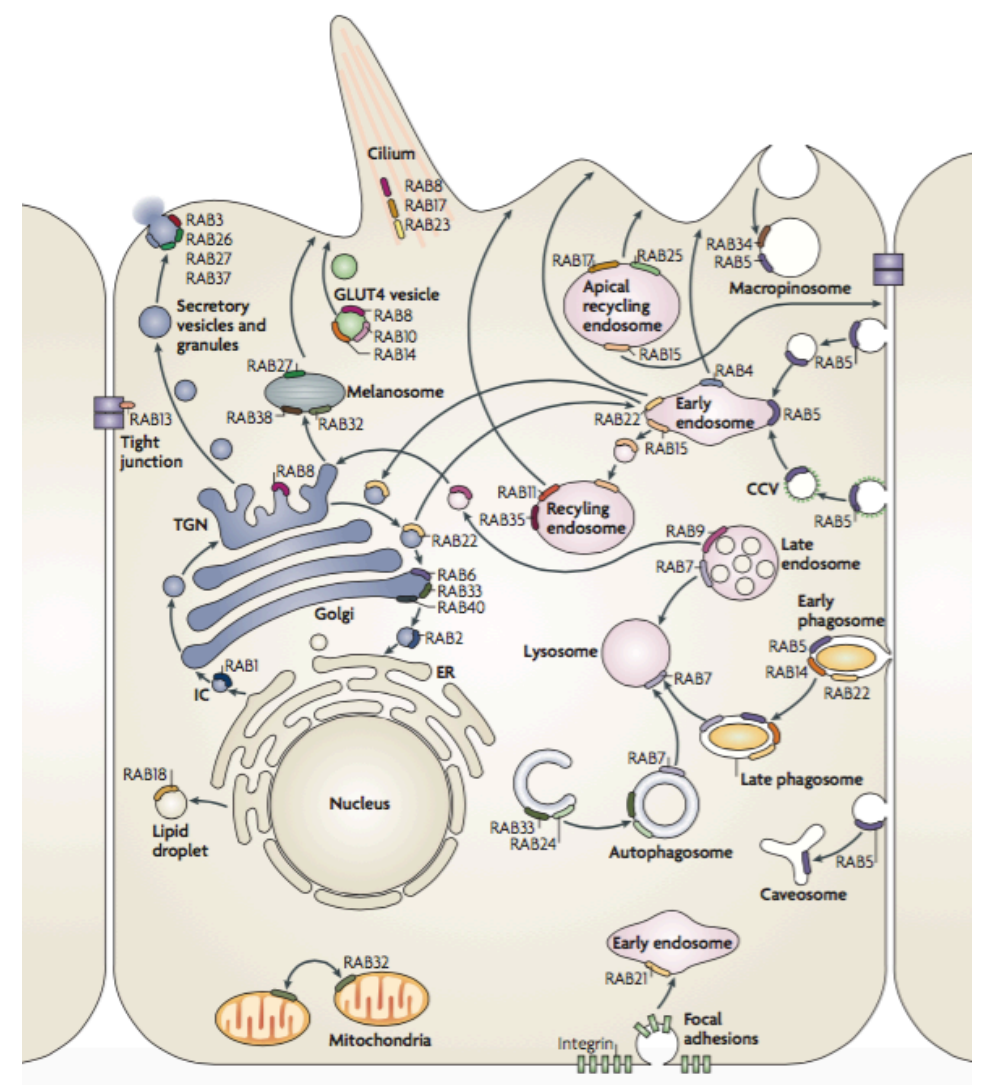

Figure 1.3. Localization and function of Rab GTPases in an epithelial cell. Reprinted by permission from Macmillan Publishers Ltd: Nature Reviews Molecular Cell Biology ${ }^{201}$, copyright 2009. http://www.nature.com/nrm/index.html

\section{Mechanisms of exocytic vesicle fusion controlled by GTPase activity}

Critical to the development of the lumen and apical membrane surface during lumen formation in both ECs and the epithelium is the fusion of vacuoles and vesicles to create a single luminal area ${ }^{11,12,36,68,69}$. Important to this process is addressing how these polarized membrane compartments are able to associate with one another and combine lipid bilayers in formation of the apical membrane surface and maintenance of 
asymmetrical cell polarity. Recently, it was reported in MDCK cyst models of epithelial lumen formation that synaptotagmin-like protein (Slp)2-a localizes to the luminal membrane in a PtdIns $(4,5) P_{2}$-dependent manner, where it functions to target Rab27 positive vesicles to initiate formation of a single lumen. Vesicle tethering to the apical membrane and fusion of vesicles is then controlled by Slp4-a in conjunction with Rab27/Rab3/Rab8 and the SNARE syntaxin- $3^{210}$. This mechanism then acts in coordination with a Rab11A/Rab8A cascade and its effectors, including the exocyst, to form a de novo apical lumen membrane by exocytic delivery and fusion of vesicles ${ }^{68}$.

Slps1-5 are Rab effectors involved in regulation of exocytosis and contain an amino-terminal Rab-binding domain i.e., the Slp homology domain, and tandem carboxyterminal $\mathrm{C} 2$ domains involved in $\mathrm{Ca}^{2+}$ and phospholipid binding, which function in tethering secretory vesicles to the plasma membrane ${ }^{211,212}$. Furthermore, these tandem C2 domains are homologous to the $\mathrm{C} 2 \mathrm{~A}$ and $\mathrm{C} 2 \mathrm{~B}$ domains of synaptotagmins, and expression of most synaptotagmins is highest in neuronal tissues ${ }^{212,213}$. Many of these synaptotagmin members function by assisting soluble $N$-ethylmaleimide-sensitive fusion protein attachment protein receptors (SNAREs) in $\mathrm{Ca}^{2+}$-dependent exocytosis ${ }^{214}$. SNARE superfamily proteins are critically involved in many intracellular membrane-fusion events and consist of SNARE motifs that comprise a highly stable four-helix bundle designated as the SNARE complex within the SNARE protein ${ }^{215,216}$. During fusion events, at least one SNARE protein that contains a transmembrane domain must be present in each of the membranes undergoing fusion with one another. The SNARE complex then forms in a trans configuration where the SNAREs involved in the formation of the complex are localized in different membranes. Then the complete SNARE complex is formed, which 
results in a cis complex in which all of the contributing SNAREs are localized in the same membrane and this complete complex formation temporally coincides with membrane fusion. The complex is then disassembled to free SNAREs for other fusion events $^{212}$

In endothelial cells, it has been reported that Rab27A associates with WPB secretory granules that are released from the endothelium into the blood in response to thrombosis and inflammation ${ }^{217,218}$. Previous work investigating the mechanisms of endothelial lumen formation reported that von Willebrand Factor (VWF), a component of $\mathrm{WPBs}^{219}$, was present in pinocytic vacuoles suggesting that WPBs may be degranulating in these vacuoles as part of an exocytic mechanism potentially involved in lumen formation ${ }^{74}$. Addressing the regulation of WPB exocytosis, it was recently demonstrated that WPBs recruit Slp4-a and this involves Rab27A and Rab3B where Slp-4a acts as a positive regulator of WPB exocytosis. However, results from this study suggest that WPB exocytosis is dependent on Rab27A in this mechanism as knockdown of Rab27A inhibited VWF secretion whereas depletion of Rab3B had no effect ${ }^{220}$. Additionally, recent evidence has demonstrated that Slp-4a is a binding partner of syntaxin-binding protein 1 (STXBP1) and the SNAREs syntaxin-2 and -3 in endothelial cells suggesting that a Rab27A-Slp-4a complex promotes WPB exocytosis through its interaction with STXBP1. In addition to Rab isoforms, the small GTPase RalA has also been reported to associate with WPBs ${ }^{221,222}$ and control its secretion through its effectors Sec5 and Exo84, which are members of the exocyst complex ${ }^{223,224}$. While it is necessary during endothelial lumen formation for trafficked membrane vacuoles/vesicles to coalesce to form a nascent lumen, the molecular mechanism of these fusion events is poorly 
understood. However, given the similarities between regulators controlling exocytic mechanisms in both epithelial and endothelial cells it is probable that these mechanisms serve a function during EC tubulogenesis.

\section{The importance of integrating in vitro and in vivo models in investigating mechanisms of vascular development and regulation}

Considerable progress has been made in our understanding of the molecular events controlling the development of the vasculature and how it is regulated postnatally, particularly in contexts of tissue injury, tumorigenesis and other diseases associated with

cardiovascular abnormalities ${ }^{8,9,11,12,69,97}$. Importantly, much of what has been elucidated and advanced the field of vascular biology has resulted from studies both in vitro and in vivo that have identified basic cellular mechanisms underlying survival, proliferation, migration, invasion and morphogenesis. As Cre recombinase and other gene editing technologies, such as CRISPR-Cas9, are becoming more advanced and specific, it has become possible to investigate and identify the functional requirement of key molecules during vascular development and regulation during postnatal life in the context of a multicellular organism. However, complications in vivo can arise from phenotypic lethality in knockout models or from the contributions of other tissues that may make it difficult to assess specific molecular mechanisms controlling vascular morphogenesis and vascular integrity/maintenance. Additionally, it is very difficult to assess the earliest events of vasculogeneis in these models. Therefore, it is critical to also utilize in vitro models under highly defined conditions, as highlighted here, in order to elucidate the 
molecular mechanisms controlling these pathways. These in vitro models offer the advantage of highly specific manipulation of molecules singly or in combination that can be observed to directly affect processes such as EC vasculogenesis, angiogenic sprouting from EC monolayers, pericyte recruitment and vascular regression ${ }^{12,69,70}$. By integrating studies using both in vitro and in vivo systems, significant advancements can be made in identifying targets for therapy in a number of different disease states.

\section{Summary}

From the onset the goal of this thesis work was to identify and determine the novel role of small GTPases of the Ras superfamily and their upstream regulators and downstream effectors during EC tubulogenesis. An extensive number of studies suggest a critical role for the Rho-family GTPases Cdc42 and Rac1 in promoting EC tubulogenesis through key downstream effectors such as PAK2, PAK4, Par3 and Par6b while activation of RhoA-ROCK activity inhibits the ability of ECs to form tubes during early morphogenesis events $56,57,76,89,145,152,154$. However, the role of other GTPases such as Ras and other Ras-related proteins that are upstream of the Raf-MEK-Erk kinase cascade or that have been implicated in stabilization of the endothelium have not been well characterized during EC tubulogenesis. To this end, we have identified novel roles for the GTPases Rac2, k-Ras, and Rap1B in addition to Cdc42 and Rac1 in EC tubulogenesis. Additionally, we identified key functional roles for the downstream effectors IQGAP1, MRCK $\beta, \beta$-Pix and GIT1 and we also have identified key negative regulators of EC tubulogenesis as the GAPs Arhgap31, a Cdc42/Rac GAP, and RASA1, a Ras GAP. 
Finally, of critical importance during EC lumen formation is understanding the mechanism of directed membrane trafficking during early morphogenesis events to form a tube structure and establish apical basal polarity; i.e. what controls generation of pinocytic intracellular vacuoles, polarized trafficking from the basal membrane to the apical membrane, fusion of the vacuoles into a lumen and finally what roles do other regulators of vesicle trafficking have during these events (Figure 1.4)? It has been observed that Rac1 ${ }^{189}$ and $\mathrm{Src}^{191}$ activity are involved in generation of macropinosomes while Cdc42 and Rac1 have been observed to label intracellular vacuole structures during EC lumen formation ${ }^{77,78,179}$. Interestingly, recombinant adenovirus expression of GFPRalA in lumen forming ECs labeled intracellular vacuoles as well ${ }^{12,70}$. Furthermore, studies have now shown a critical role for both Rab11A and Rab8A during epithelial morphogenesis by demonstrating that these GTPases were necessary for proper trafficking of vesicles to the apical membrane initiation site ${ }^{62,68,205-209}$. Therefore it may be hypothesized that key regulators of EC lumen morphogenesis, such as Cdc42, Rac1 and Src may be required for generation of intracellular vacuoles while Rab and Ral GTPase activity may function to direct trafficking of these vacuoles and other vesicles to the proper site and may control the fusion of vacuoles/vesicles to form a lumen. To address this, we have performed a siRNA-mediated knockdown screen investigating the roles of Rab and Ral GTPases, which are primarily involved in regulation of endocytic and exocytic membrane trafficking events, during early EC tubulogenesis as well as caveolin-1, which is associated with caveolae. Additionally, we also developed a number of recombinant-adenovirus tools that express fluorescent fusion proteins that were used in combination with confocal microscopy analysis to assess the localization of key 
molecules to vacuoles, vesicles and the apical membrane surface during EC lumen morphogenesis. With these studies and additional studies utilizing chemical inhibitors, we address the roles of critical regulators of intracellular vacuole formation, polarized trafficking and vacuole/vesicle fusion events in controlling EC tubulogenesis in ongoing studies.

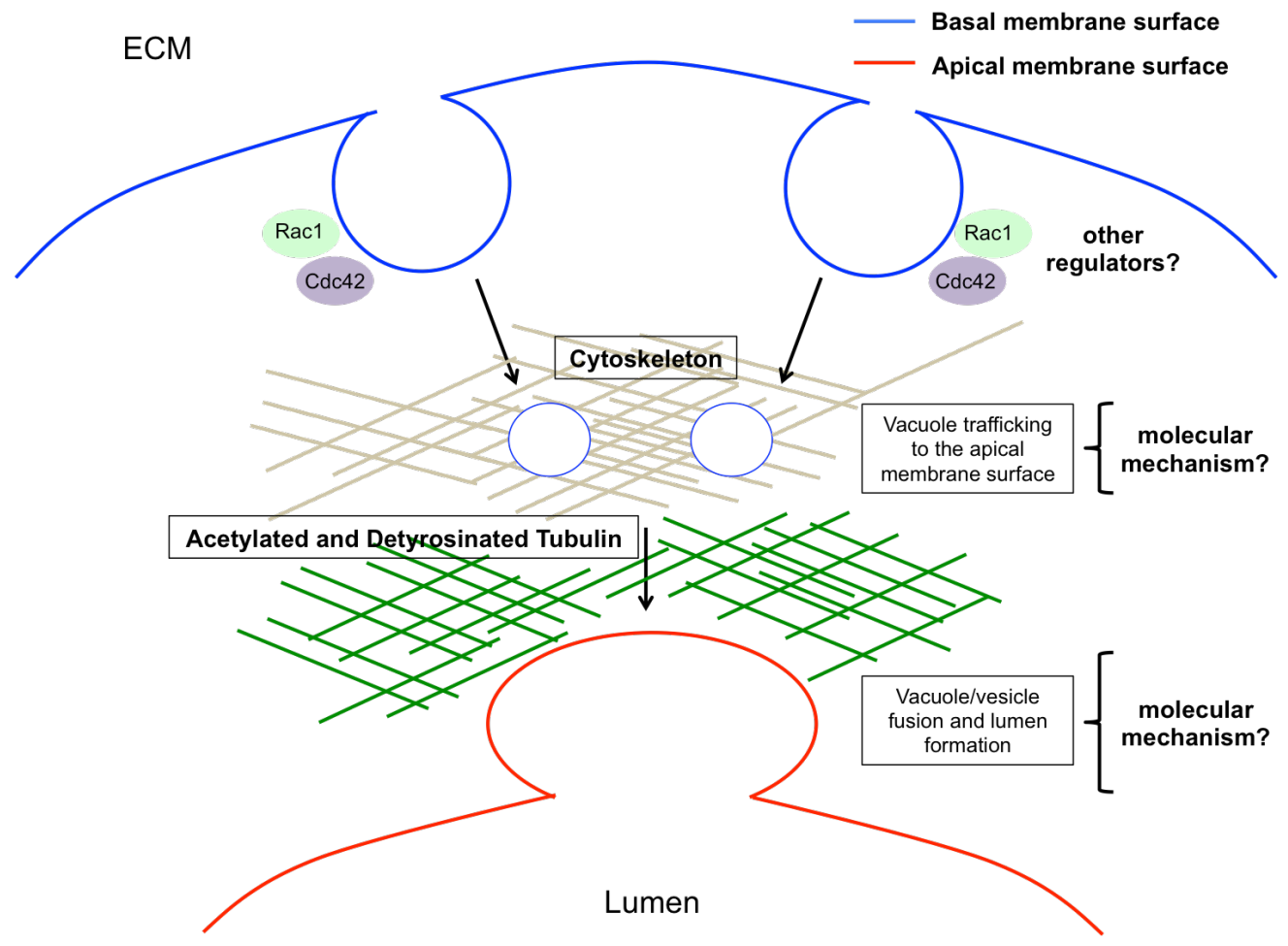

Figure 1.4. Polarized trafficking and fusion of pinocytic intracellular vacuoles and membrane vesicles creates the apical luminal area during endothelial tubulogenesis. 


\section{REFERENCES}

1. Risau, W. and I. Flamme, Vasculogenesis. Annual Review of Cell and Developmental Biology, 1995. 11(1): p. 73-91.

2. Baldwin, H.S., Early embryonic vascular development. Cardiovasc Res, 1996. 31 Spec No: p. E34-45.

3. Flamme, I., T. Frölich, and W. Risau, Molecular mechanisms of vasculogenesis and embryonic angiogenesis. Journal of Cellular Physiology, 1997. 173(2): p. 206-210.

4. Drake, C.J. and P.A. Fleming, Vasculogenesis in the day 6.5 to 9.5 mouse embryo. Blood, 2000. 95(5): p. 1671-9.

5. $\quad$ Folkman, J. and C. Haudenschild, Angiogenesis in vitro. Nature, 1980. 288(5791): p. 551-556.

6. Hanahan, D., Signaling Vascular Morphogenesis and Maintenance. Science, 1997. 277(5322): p. 48-50.

7. Jain, R.K., Molecular regulation of vessel maturation. Nat Med, 2003. 9(6): p. 685-693.

8. Carmeliet, P., Angiogenesis in life, disease and medicine. Nature, 2005. 438(7070): p. 932-936.

9. Adams, R.H. and K. Alitalo, Molecular regulation of angiogenesis and lymphangiogenesis. Nat Rev Mol Cell Biol, 2007. 8(6): p. 464-478.

10. Koh, W., et al., Chapter 5 In Vitro Three Dimensional Collagen Matrix Models of Endothelial Lumen Formation During Vasculogenesis and Angiogenesis, in Methods in Enzymology, A.C. David, Editor. 2008, Academic Press. p. 83-101.

11. $\mathrm{Xu}, \mathrm{K}$. and $\mathrm{O}$. Cleaver, Tubulogenesis during blood vessel formation. Seminars in Cell \& Developmental Biology, 2011. 22(9): p. 993-1004.

12. Sacharidou, A., A.N. Stratman, and G.E. Davis, Molecular Mechanisms Controlling Vascular Lumen Formation in Three-Dimensional Extracellular Matrices. Cells Tissues Organs, 2012. 195(1-2): p. 122-143. 
13. Sigurbjornsdottir, S., R. Mathew, and M. Leptin, Molecular mechanisms of de novo lumen formation. Nat Rev Mol Cell Biol, 2014. advance online

publication.

14. Drake, C.J., P.A. Fleming, and W.S. Argraves, The genetics of vasculogenesis. Novartis Found Symp, 2007. 283: p. 61-71; discussion 71-6, 238-41.

15. Kim, D.J., L.A. Martinez-Lemus, and G.E. Davis, EB1, p150Glued, and Clasp1 control endothelial tubulogenesis through microtubule assembly, acetylation, and apical polarization. Blood Vol. 121. 2013. 3521-3530.

16. Davis, G., et al., Endothelial Cell Polarization During Lumen Formation, Tubulogenesis, and Vessel Maturation in 3D Extracellular Matrices, in Cell Polarity 1, K. Ebnet, Editor. 2015, Springer International Publishing. p. 205-220.

17. Daniel, T.O. and D. Abrahamson, Endothelial Signal Integration in Vascular Assembly. Annual Review of Physiology, 2000. 62(1): p. 649-671.

18. Eichmann, A., et al., Vascular development: from precursor cells to branched arterial and venous networks. Int J Dev Biol, 2005. 49(2-3): p. 259-67.

19. Armulik, A., A. Abramsson, and C. Betsholtz, Endothelial/Pericyte Interactions. Circulation Research, 2005. 97(6): p. 512-523.

20. Bergers, G. and S. Song, The role of pericytes in blood-vessel formation and maintenance. Neuro-Oncology, 2005. 7(4): p. 452-464.

21. Stratman, A.N., et al., Pericyte recruitment during vasculogenic tube assembly stimulates endothelial basement membrane matrix formation. Vol. 114. 2009. 5091-5101.

22. Stratman, A.N., et al., Endothelial-derived PDGF-BB and HB-EGF coordinately regulate pericyte recruitment during vasculogenic tube assembly and stabilization. Vol. 116. 2010. 4720-4730.

23. Stratman, A.N. and G.E. Davis, Endothelial Cell-Pericyte Interactions Stimulate Basement Membrane Matrix Assembly: Influence on Vascular Tube Remodeling, Maturation, and Stabilization. Microscopy and Microanalysis, 2012. 18(01): p. 68-80.

24. Davis, G.E., et al., Control of vascular tube morphogenesis and maturation in 3D extracellular matrices by endothelial cells and pericytes. Methods Mol Biol, 2013. 1066: p. 17-28. 
25. Drake, C.J., Embryonic and adult vasculogenesis. Birth Defects Research Part C: Embryo Today: Reviews, 2003. 69(1): p. 73-82.

26. Reynolds, L.P., S.D. Killilea, and D.A. Redmer, Angiogenesis in the female reproductive system. The FASEB Journal, 1992. 6(3): p. 886-92.

27. Demir, R., A. Yaba, and B. Huppertz, Vasculogenesis and angiogenesis in the endometrium during menstrual cycle and implantation. Acta Histochem, 2010. 112(3): p. 203-14.

28. Davis, G.E., et al., Regulation of tissue injury responses by the exposure of matricryptic sites within extracellular matrix molecules. Am J Pathol, 2000. 156(5): p. 1489-98.

29. Tonnesen, M.G., X. Feng, and R.A.F. Clark, Angiogenesis in Wound Healing. J Investig Dermatol Symp Proc, 2000. 5(1): p. 40-46.

30. Jain, R.K., Normalization of tumor vasculature: an emerging concept in antiangiogenic therapy. Science, 2005. 307(5706): p. 58-62.

31. Chung, A.S., J. Lee, and N. Ferrara, Targeting the tumour vasculature: insights from physiological angiogenesis. Nat Rev Cancer, 2010. 10(7): p. 505-514.

32. Timar, J., et al., Angiogenesis-dependent diseases and angiogenesis therapy. Pathol Oncol Res, 2001. 7(2): p. 85-94.

33. Brouillard, P. and M. Vikkula, Genetic causes of vascular malformations. Human Molecular Genetics, 2007. 16(R2): p. R140-R149.

34. Paneni, F., et al., Diabetes and vascular disease: pathophysiology, clinical consequences, and medical therapy: part I. European Heart Journal, 2013. 34(31): p. 2436-2443.

35. Davis, G.E., P.R. Norden, and S.L.K. Bowers, Molecular control of capillary morphogenesis and maturation by recognition and remodeling of the extracellular matrix: functional roles of endothelial cells and pericytes in health and disease. Connective Tissue Research, 2015: p. 1-11.

36. Bryant, D.M. and K.E. Mostov, From cells to organs: building polarized tissue. Nat Rev Mol Cell Biol, 2008. 9(11): p. 887-901.

37. Li, R. and G.G. Gundersen, Beyond polymer polarity: how the cytoskeleton builds a polarized cell. Nat Rev Mol Cell Biol, 2008. 9(11): p. 860-873. 
38. Wegner, A. and J. Engel, Kinetics of the cooperative association of actin to actin filament. Biophysical Chemistry, 1975. 3(3): p. 215-225.

39. Barton, J.S. and G.H. Riazi, Evidence for two growth steps in microtubule polymerization. Biochim Biophys Acta, 1980. 630(3): p. 392-401.

40. Ridley, A.J., Rho GTPases and actin dynamics in membrane protrusions and vesicle trafficking. Trends Cell Biol, 2006. 16(10): p. 522-9.

41. Pollard, T.D., Regulation of actin filament assembly by Arp2/3 complex and formins. Annu Rev Biophys Biomol Struct, 2007. 36: p. 451-77.

42. Gundersen, G.G., E.R. Gomes, and Y. Wen, Cortical control of microtubule stability and polarization. Current Opinion in Cell Biology, 2004. 16(1): p. 106112.

43. Lansbergen, G. and A. Akhmanova, Microtubule plus end: a hub of cellular activities. Traffic, 2006. 7(5): p. 499-507.

44. Larcher, J.-C., et al., Interaction of Kinesin Motor Domains with $\alpha$ - and $\beta$-Tubulin Subunits at a Tau-independent Binding Site: REGULATION BY POLYGLUTAMYLATION. Journal of Biological Chemistry, 1996. 271(36): p. 22117-22124.

45. Liao, G. and G.G. Gundersen, Kinesin is a candidate for cross-bridging microtubules and intermediate filaments. Selective binding of kinesin to detyrosinated tubulin and vimentin. J Biol Chem, 1998. 273(16): p. 9797-803.

46. Lin, S.X., G.G. Gundersen, and F.R. Maxfield, Export from pericentriolar endocytic recycling compartment to cell surface depends on stable, detyrosinated (glu) microtubules and kinesin. Mol Biol Cell, 2002. 13(1): p. 96-109.

47. Reed, N.A., et al., Microtubule acetylation promotes kinesin-1 binding and transport. Curr Biol, 2006. 16(21): p. 2166-72.

48. Etienne-Manneville, S. and A. Hall, Integrin-mediated activation of Cdc42 controls cell polarity in migrating astrocytes through PKCzeta. Cell, 2001. 106(4): p. 489-98.

49. Palazzo, A.F., et al., Cdc42, dynein, and dynactin regulate MTOC reorientation independent of Rho-regulated microtubule stabilization. Current Biology, 2001. 11(19): p. 1536-1541. 
50. Solecki, D.J., et al., Par6alpha signaling controls glial-guided neuronal migration. Nat Neurosci, 2004. 7(11): p. 1195-203.

51. Watanabe, T., et al., Interaction with IQGAP1 links APC to Rac1, Cdc42, and actin filaments during cell polarization and migration. Dev Cell, 2004. 7(6): p. 871-83.

52. Gomes, E.R., S. Jani, and G.G. Gundersen, Nuclear movement regulated by Cdc42, MRCK, myosin, and actin flow establishes MTOC polarization in migrating cells. Cell, 2005. 121(3): p. 451-63.

53. Yamana, N., et al., The Rho-mDial pathway regulates cell polarity and focal adhesion turnover in migrating cells through mobilizing Apc and c-Src. Mol Cell Biol, 2006. 26(18): p. 6844-58.

54. Iruela-Arispe, M.L. and G.E. Davis, Cellular and Molecular Mechanisms of Vascular Lumen Formation. Developmental Cell, 2009. 16(2): p. 222-231.

55. Martin-Belmonte, F., et al., PTEN-Mediated Apical Segregation of Phosphoinositides Controls Epithelial Morphogenesis through Cdc42. Cell, 2007. 128(2): p. 383-397.

56. Koh, W., R.D. Mahan, and G.E. Davis, Cdc42- and Rac1-mediated endothelial lumen formation requires Pak2, Pak4 and Par3, and PKC-dependent signaling. Journal of Cell Science, 2008. 121(7): p. 989-1001.

57. Sacharidou, A., et al., Endothelial lumen signaling complexes control 3D matrixspecific tubulogenesis through interdependent Cdc42-and MT1-MMP-mediated events. Vol. 115. 2010. 5259-5269.

58. Gory-Faure, S., et al., Role of vascular endothelial-cadherin in vascular morphogenesis. Development, 1999. 126(10): p. 2093-102.

59. Iden, S., et al., A distinct PAR complex associates physically with VE-cadherin in vertebrate endothelial cells. EMBO Rep, 2006. 7(12): p. 1239-46.

60. Strilić, B., et al., The Molecular Basis of Vascular Lumen Formation in the Developing Mouse Aorta. Developmental Cell, 2009. 17(4): p. 505-515.

61. Lubarsky, B. and M.A. Krasnow, Tube Morphogenesis: Making and Shaping Biological Tubes. Cell, 2003. 112(1): p. 19-28.

62. Datta, A., D.M. Bryant, and K.E. Mostov, Molecular Regulation of Lumen Morphogenesis. Current Biology, 2011. 21(3): p. R126-R136. 
63. Gore, A.V., et al., Vascular Development in the Zebrafish. Cold Spring Harbor Perspectives in Medicine, 2012. 2(5).

64. O'Brien, L.E., et al., Racl orientates epithelial apical polarity through effects on basolateral laminin assembly. Nat Cell Biol, 2001. 3(9): p. 831-838.

65. Yu, W., et al., 31 -Integrin Orients Epithelial Polarity via Rac1 and Laminin. Molecular Biology of the Cell, 2005. 16(2): p. 433-445.

66. Rodríguez-Fraticelli, A.E., M. Gálvez-Santisteban, and F. Martín-Belmonte, Divide and polarize: recent advances in the molecular mechanism regulating epithelial tubulogenesis. Current Opinion in Cell Biology, 2011. 23(5): p. 638646.

67. Gassama-Diagne, A., et al., Phosphatidylinositol-3,4,5-trisphosphate regulates the formation of the basolateral plasma membrane in epithelial cells. Nat Cell Biol, 2006. 8(9): p. 963-70.

68. Bryant, D.M., et al., A molecular network for de novo generation of the apical surface and lumen. Nat Cell Biol, 2010. 12(11): p. 1035-1045.

69. Davis, G.E., et al., Chapter Three - Molecular Basis for Endothelial Lumen Formation and Tubulogenesis During Vasculogenesis and Angiogenic Sprouting, in International Review of Cell and Molecular Biology, W.J. Kwang, Editor. 2011, Academic Press. p. 101-165.

70. Davis, G.E., W. Koh, and A.N. Stratman, Mechanisms controlling human endothelial lumen formation and tube assembly in three-dimensional extracellular matrices. Birth Defects Research Part C: Embryo Today: Reviews, 2007. 81(4): p. 270-285.

71. Davis, G., Molecular Regulation of Vasculogenesis and Angiogenesis: Recent Advances and Future Directions, in Molecular and Translational Vascular Medicine, J.W. Homeister and M.S. Willis, Editors. 2012, Humana Press. p. 169206.

72. O'Brien, L.E., et al., Morphological and biochemical analysis of Rac1 in threedimensional epithelial cell cultures. Methods Enzymol, 2006. 406: p. 676-91.

73. Stratman, A.N., M.J. Davis, and G.E. Davis, VEGF and FGF prime vascular tube morphogenesis and sprouting directed by hematopoietic stem cell cytokines. Blood Vol. 117. 2011. 3709-3719. 
74. Davis, G. and C. Camarillo, An alpha 2 beta 1 integrin-dependent pinocytic mechanism involving intracellular vacuole formation and coalescence regulates capillary lumen and tube formation in three-dimensional collagen matrix. Exp Cell Res, 1996. 224(1): p. 39 - 51.

75. Bayless, K.J., R. Salazar, and G.E. Davis, RGD-dependent vacuolation and lumen formation observed during endothelial cell morphogenesis in three-dimensional fibrin matrices involves the alpha(v)beta(3) and alpha(5)beta(1) integrins. Am J Pathol, 2000. 156(5): p. 1673-83.

76. Bayless, K. and G. Davis, The Cdc42 and Racl GTPases are required for capillary lumen formation in three-dimensional extracellular matrices. J Cell Sci, 2002. 115(6): p. 1123 - 1136.

77. Davis, G.E. and K.J. Bayless, An Integrin and Rho GTPase-Dependent Pinocytic Vacuole Mechanism Controls Capillary Lumen Formation in Collagen and Fibrin Matrices. Microcirculation, 2003. 10(1): p. 27-44.

78. Kamei, M., et al., Endothelial tubes assemble from intracellular vacuoles in vivo. Nature, 2006. 442(7101): p. 453-456.

79. Liu, H., et al., Ccm1 regulates microvascular morphogenesis during angiogenesis. J Vasc Res, 2011. 48(2): p. 130-40.

80. Yu, J.A., et al., Single cell analysis of endothelial morphogenesis in vivo. Development, 2015.

81. Drake, C.J., L.A. Davis, and C.D. Little, Antibodies to beta 1-integrins cause alterations of aortic vasculogenesis, in vivo. Dev Dyn, 1992. 193(1): p. 83-91.

82. Hynes, R.O., B.L. Bader, and K. Hodivala-Dilke, Integrins in vascular development. Braz J Med Biol Res, 1999. 32(5): p. 501-10.

83. Bell, S.E., et al., Differential gene expression during capillary morphogenesis in $3 D$ collagen matrices: regulated expression of genes involved in basement membrane matrix assembly, cell cycle progression, cellular differentiation and $G$ protein signaling. J Cell Sci, 2001. 114(Pt 15): p. 2755-73.

84. Kazemi, S., et al., Differential role of bFGF and VEGF for vasculogenesis. Cell Physiol Biochem, 2002. 12(2-3): p. 55-62.

85. Davis, G.E. and D.R. Senger, Endothelial Extracellular Matrix: Biosynthesis, Remodeling, and Functions During Vascular Morphogenesis and Neovessel Stabilization. Circulation Research, 2005. 97(11): p. 1093-1107. 
86. Stratman, A.N., et al., Endothelial cell lumen and vascular guidance tunnel formation requires MT1-MMP-dependent proteolysis in 3-dimensional collagen matrices. Vol. 114. 2009. 237-247.

87. Zovein, A.C., et al., $\beta 1$ Integrin Establishes Endothelial Cell Polarity and Arteriolar Lumen Formation via a Par3-Dependent Mechanism. Developmental Cell, 2010. 18(1): p. 39-51.

88. Smith, A.O., et al., Hematopoietic Stem Cell Cytokines and Fibroblast Growth factor-2 Stimulate Human Endothelial Cell-Pericyte Tube Co-Assembly in 3D Fibrin Matrices under Serum-Free Defined Conditions. PLoS ONE, 2013. 8(12): p. e85147.

89. Koh, W., et al., Formation of endothelial lumens requires a coordinated PKCE-, Src-, Pak- and Raf-kinase-dependent signaling cascade downstream of Cdc42 activation. Journal of Cell Science, 2009. 122(11): p. 1812-1822.

90. Rozario, T. and D.W. DeSimone, The extracellular matrix in development and morphogenesis: A dynamic view. Developmental Biology, 2010. 341(1): p. 126140.

91. Hynes, R.O., Cell-matrix adhesion in vascular development. Journal of Thrombosis and Haemostasis, 2007. 5: p. 32-40.

92. Bayless, K. and G. Davis, Sphingosine 1-phosphate markedly induces matrix metalloproteinase and integrin-dependent human endothelial cell invasion and lumen formation in three-dimensional collagen and fibrin matrices. Biochem Biophys Res Commun, 2003. 312(4): p. 903 - 913.

93. Tarone, G., et al., Integrin function and regulation in development. Int J Dev Biol, 2000. 44(6): p. 725-31.

94. Humphries, J.D., A. Byron, and M.J. Humphries, Integrin ligands at a glance. Journal of Cell Science, 2006. 119(19): p. 3901-3903.

95. Senger, D.R., et al., Angiogenesis promoted by vascular endothelial growth factor: regulation through alphalbetal and alpha2betal integrins. Proc Natl Acad Sci U S A, 1997. 94(25): p. 13612-7.

96. Gerhardt, H., et al., VEGF guides angiogenic sprouting utilizing endothelial tip cell filopodia. J Cell Biol, 2003. 161(6): p. 1163-77. 
97. Holderfield, M.T. and C.C.W. Hughes, Crosstalk Between Vascular Endothelial Growth Factor, Notch, and Transforming Growth Factor- $\beta$ in Vascular Morphogenesis. Circulation Research, 2008. 102(6): p. 637-652.

98. Horowitz, A. and M. Simons, Branching Morphogenesis. Circulation Research, 2008. 103(8): p. 784-795.

99. Lohela, M., et al., VEGFs and receptors involved in angiogenesis versus lymphangiogenesis. Curr Opin Cell Biol, 2009. 21(2): p. 154-65.

100. Murakami, M. and M. Simons, Fibroblast growth factor regulation of neovascularization. Curr Opin Hematol, 2008. 15(3): p. 215-20.

101. Beenken, A. and M. Mohammadi, The FGF family: biology, pathophysiology and therapy. Nat Rev Drug Discov, 2009. 8(3): p. 235-53.

102. Andre, C., et al., c-kit $m R N A$ expression in human and murine hematopoietic cell lines. Oncogene, 1989. 4(8): p. 1047-9.

103. Aye, M.T., et al., Expression of stem cell factor and c-kit mRNA in cultured endothelial cells, monocytes and cloned human bone marrow stromal cells (CFU$R F)$. Exp Hematol, 1992. 20(4): p. 523-7.

104. Yamaguchi, H., et al., Umbilical vein endothelial cells are an important source of c-kit and stem cell factor which regulate the proliferation of haemopoietic progenitor cells. Br J Haematol, 1996. 94(4): p. 606-11.

105. Gilfillan, A.M. and C. Tkaczyk, Integrated signalling pathways for mast-cell activation. Nat Rev Immunol, 2006. 6(3): p. 218-230.

106. Korpelainen, E.I., et al., The receptor for interleukin 3 is selectively induced in human endothelial cells by tumor necrosis factor alpha and potentiates interleukin 8 secretion and neutrophil transmigration. Proc Natl Acad Sci U S A, 1993. 90(23): p. 11137-41.

107. Hara, T. and A. Miyajima, Function and signal transduction mediated by the interleukin 3 receptor system in hematopoiesis. Stem Cells, 1996. 14(6): p. 60518.

108. Hembruff, S.L. and N. Cheng, Chemokine signaling in cancer: Implications on the tumor microenvironment and therapeutic targeting. Cancer Ther, 2009. 7(A): p. 254-67. 
109. Saunders, W.B., K.J. Bayless, and G.E. Davis, MMP-1 activation by serine proteases and MMP-10 induces human capillary tubular network collapse and regression in $3 D$ collagen matrices. Journal of Cell Science, 2005. 118(10): p. 2325-2340.

110. Saunders, W.B., et al., Coregulation of vascular tube stabilization by endothelial cell TIMP-2 and pericyte TIMP-3. The Journal of Cell Biology, 2006. 175(1): p. 179-191.

111. Page-McCaw, A., A.J. Ewald, and Z. Werb, Matrix metalloproteinases and the regulation of tissue remodelling. Nat Rev Mol Cell Biol, 2007. 8(3): p. 221-233.

112. Zhou, Z., et al., Impaired endochondral ossification and angiogenesis in mice deficient in membrane-type matrix metalloproteinase I. Proc Natl Acad Sci U S A, 2000. 97(8): p. 4052-7.

113. Eliceiri, B.P., et al., Src-mediated coupling of focal adhesion kinase to integrin $\alpha v \beta 5$ in vascular endothelial growth factor signaling. The Journal of Cell Biology, 2002. 157(1): p. 149-160.

114. Playford, M.P. and M.D. Schaller, The interplay between Src and integrins in normal and tumor biology. Oncogene, 2004. 23(48): p. 7928-7946.

115. LIU, Y. and D.R. SENGER, Matrix-specific activation of Src and Rho initiates capillary morphogenesis of endothelial cells. The FASEB Journal, 2004. 18(3): p. 457-468.

116. Thomas, S.M. and J.S. Brugge, Cellular Functions Regulated by Src Family Kinases. Annual Review of Cell and Developmental Biology, 1997. 13(1): p. 513609.

117. Im, E. and A. Kazlauskas, Src Family Kinases Promote Vessel Stability by Antagonizing the Rho/ROCK Pathway. Journal of Biological Chemistry, 2007. 282(40): p. 29122-29129.

118. Eliceiri, B.P., et al., Selective Requirement for Src Kinases during VEGF-Induced Angiogenesis and Vascular Permeability. Molecular Cell, 1999. 4(6): p. 915-924.

119. Hood, J.D., et al., Differential alphav integrin-mediated Ras-ERK signaling during two pathways of angiogenesis. J Cell Biol, 2003. 162(5): p. 933-43.

120. Steinberg, S.F., Structural Basis of Protein Kinase C Isoform Function. Physiological Reviews, 2008. 88(4): p. 1341-1378. 
121. Montesano, R. and L. Orci, Tumor-promoting phorbol esters induce angiogenesis in vitro. Cell, 1985. 42(2): p. 469-477.

122. Montesano, R. and L. Orci, Intracellular diaphragmed fenestrae in cultured capillary endothelial cells. Journal of Cell Science, 1988. 89(3): p. 441-447.

123. Taylor, C.J., K. Motamed, and B. Lilly, Protein kinase $C$ and downstream signaling pathways in a three-dimensional model of phorbol ester-induced angiogenesis. Angiogenesis, 2006. 9(2): p. 39-51.

124. Morris, P.B., et al., Tumor-promoting phorbol esters induce angiogenesis in vivo. Am J Physiol, 1988. 254(2 Pt 1): p. C318-22.

125. Colicelli, J., Human RAS Superfamily Proteins and Related GTPases. Science's STKE : signal transduction knowledge environment, 2004. 2004(250): p. RE13RE13.

126. Wennerberg, K., K.L. Rossman, and C.J. Der, The Ras superfamily at a glance. Journal of Cell Science, 2005. 118(5): p. 843-846.

127. Seabra, M.C. and C. Wasmeier, Controlling the location and activation of Rab GTPases. Current Opinion in Cell Biology, 2004. 16(4): p. 451-457.

128. Hall, A., Rho family GTPases. Vol. 40. 2012. 1378-1382.

129. Bishop, A.L. and A. Hall, Rho GTPases and their effector proteins. Biochem J, 2000. 348 Pt 2: p. 241-55.

130. Cherfils, J. and M. Zeghouf, Regulation of Small GTPases by GEFs, GAPs, and GDIs. Vol. 93. 2013. 269-309.

131. Ridley, A.J. and A. Hall, The small GTP-binding protein rho regulates the assembly of focal adhesions and actin stress fibers in response to growth factors. Cell, 1992. 70(3): p. 389-99.

132. Ridley, A.J., et al., The small GTP-binding protein rac regulates growth factorinduced membrane ruffling. Cell, 1992. 70(3): p. 401-10.

133. Nobes, C.D. and A. Hall, Rho, rac, and cdc42 GTPases regulate the assembly of multimolecular focal complexes associated with actin stress fibers, lamellipodia, and filopodia. Cell, 1995. 81(1): p. 53-62. 
134. Kozma, R., et al., The Ras-related protein Cdc42Hs and bradykinin promote formation of peripheral actin microspikes and filopodia in Swiss 3 T3 fibroblasts. Mol Cell Biol, 1995. 15(4): p. 1942-52.

135. Sugihara, K., et al., Racl is required for the formation of three germ layers during gastrulation. Oncogene, 1998. 17(26): p. 3427-33.

136. Chen, F., et al., Cdc42 is required for PIP(2)-induced actin polymerization and early development but not for cell viability. Curr Biol, 2000. 10(13): p. 758-65.

137. Manser, E., et al., A brain serine/threonine protein kinase activated by Cdc42 and Rac1. Nature, 1994. 367(6458): p. 40-6.

138. Kimura, K., et al., Regulation of myosin phosphatase by Rho and Rho-associated kinase (Rho-kinase). Science, 1996. 273(5272): p. 245-8.

139. Watanabe, N., et al., Cooperation between mDial and ROCK in Rho-induced actin reorganization. Nat Cell Biol, 1999. 1(3): p. 136-143.

140. Joberty, G., et al., The cell-polarity protein Par6 links Par3 and atypical protein kinase C to Cdc42. Nat Cell Biol, 2000. 2(8): p. 531-539.

141. Lin, D., et al., A mammalian PAR-3-PAR-6 complex implicated in Cdc42/Rac1 and aPKC signalling and cell polarity. Nat Cell Biol, 2000. 2(8): p. 540-547.

142. Etienne-Manneville, S. and A. Hall, Integrin-Mediated Activation of Cdc42

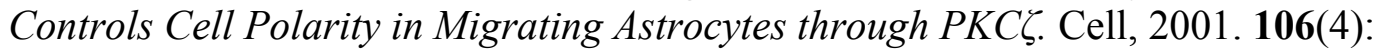
p. 489-498.

143. Ohno, S., Intercellular junctions and cellular polarity: the PAR-aPKC complex, a conserved core cassette playing fundamental roles in cell polarity. Curr Opin Cell Biol, 2001. 13(5): p. 641-8.

144. Etienne-Manneville, S. and A. Hall, Cdc42 regulates GSK-3beta and adenomatous polyposis coli to control cell polarity. Nature, 2003. 421(6924): p. 753-6.

145. Barry, D.M., et al., Cdc42 is required for cytoskeletal support of endothelial cell adhesion during blood vessel formation. Development, 2015.

146. Tan, W., et al., An essential role for Racl in endothelial cell function and vascular development. Faseb j, 2008. 22(6): p. 1829-38. 
147. Martín-Belmonte, F., et al., Cell-Polarity Dynamics Controls the Mechanism of Lumen Formation in Epithelial Morphogenesis. Current Biology, 2008. 18(7): p. 507-513.

148. Myllymäki, S.M., T.P. Teräväinen, and A. Manninen, Two Distinct IntegrinMediated Mechanisms Contribute to Apical Lumen Formation in Epithelial Cells. PLoS ONE, 2011. 6(5): p. e19453.

149. Kesavan, G., et al., Cdc42-Mediated Tubulogenesis Controls Cell Specification. Cell, 2009. 139(4): p. 791-801.

150. Yu, W.S.A.M.B.P.E.D.J.D.A.B.D.M.F.Q.W.W.W.A.Z.M.M.P.M.K.E., Involvement of RhoA, ROCK I and myosin II in inverted orientation of epithelial polarity. EMBO reports, 2008. 9(9): p. 923-929.

151. Mertens, A.E., et al., The Rac activator Tiam 1 controls tight junction biogenesis in keratinocytes through binding to and activation of the Par polarity complex. $\mathrm{J}$ Cell Biol, 2005. 170(7): p. 1029-37.

152. Bayless, K.J. and G.E. Davis, Microtubule Depolymerization Rapidly Collapses Capillary Tube Networks in Vitro and Angiogenic Vessels in Vivo through the Small GTPase Rho. Journal of Biological Chemistry, 2004. 279(12): p. 1168611695.

153. Mavria, G., et al., ERK-MAPK signaling opposes Rho-kinase to promote endothelial cell survival and sprouting during angiogenesis. Cancer Cell, 2006. 9(1): p. 33-44.

154. Xu, K., et al., Blood Vessel Tubulogenesis Requires Rasip1 Regulation of GTPase Signaling. Developmental Cell, 2011. 20(4): p. 526-539.

155. Bryant, D.M., et al., A molecular switch for the orientation of epithelial cell polarization. Dev Cell, 2014. 31(2): p. 171-87.

156. Yuan, L., et al., RhoJ is an endothelial cell-restricted Rho GTPase that mediates vascular morphogenesis and is regulated by the transcription factor ERG. Vol. 118. 2011. 1145-1153.

157. Chrzanowska-Wodnicka, M., Distinct functions for Rap1 signaling in vascular morphogenesis and dysfunction. Exp Cell Res, 2013. 319(15): p. 2350-9.

158. Chrzanowska-Wodnicka, M., et al., Raplb is required for normal platelet function and hemostasis in mice. J Clin Invest, 2005. 115(3): p. 680-7. 
159. Li, Y., et al., Rapla Null Mice Have Altered Myeloid Cell Functions Suggesting Distinct Roles for the Closely Related Rapla and $1 b$ Proteins. The Journal of Immunology, 2007. 179(12): p. 8322-8331.

160. Chrzanowska-Wodnicka, M., et al., Defective angiogenesis, endothelial migration, proliferation, and MAPK signaling in Raplb-deficient mice. Blood, 2008. 111(5): p. 2647-2656.

161. Yan, J., et al., Rapla Is a Key Regulator of Fibroblast Growth Factor 2-Induced Angiogenesis and Together with Rap1b Controls Human Endothelial Cell Functions. Molecular and Cellular Biology, 2008. 28(18): p. 5803-5810.

162. Lakshmikanthan, S., et al., Rap1 promotes VEGFR2 activation and angiogenesis by a mechanism involving integrin $\alpha v \beta 3$. Blood, 2011. 118(7): p. 2015-2026.

163. Gore, A.V., et al., Combinatorial interaction between CCM pathway genes precipitates hemorrhagic stroke. Disease Models and Mechanisms, 2008. 1(4-5): p. $275-281$.

164. Tawa, H., et al., Role of Afadin in Vascular Endothelial Growth Factor-and Sphingosine 1-Phosphate-Induced Angiogenesis. Circulation Research, 2010. 106(11): p. 1731-1742.

165. Serebriiskii, I., et al., Association of Krev-1/rapla with Krit1, a novel ankyrin repeat-containing protein encoded by a gene mapping to 7q21-22. Oncogene, 1997. 15(9): p. 1043-9.

166. Revencu, N. and M. Vikkula, Cerebral cavernous malformation: new molecular and clinical insights. Journal of Medical Genetics, 2006. 43(9): p. 716-721.

167. Laberge-le Couteulx, S., et al., Truncating mutations in CCM1, encoding KRIT1, cause hereditary cavernous angiomas. Nat Genet, 1999. 23(2): p. 189-93.

168. Sahoo, T., et al., Mutations in the Gene Encoding KRIT1, a Krev-1/rapla Binding Protein, Cause Cerebral Cavernous Malformations (CCM1). Human Molecular Genetics, 1999. 8(12): p. 2325-2333.

169. Liquori, C.L., et al., Mutations in a Gene Encoding a Novel Protein Containing a Phosphotyrosine-Binding Domain Cause Type 2 Cerebral Cavernous Malformations. The American Journal of Human Genetics, 2003. 73(6): p. 14591464.

170. Uhlik, M.T., et al., Rac-MEKK3-MKK3 scaffolding for p38 MAPK activation during hyperosmotic shock. Nat Cell Biol, 2003. 5(12): p. 1104-1110. 
171. Bergametti, F., et al., Mutations within the Programmed Cell Death 10 Gene Cause Cerebral Cavernous Malformations. The American Journal of Human Genetics, 2005. 76(1): p. 42-51.

172. Li, X., et al., Structural Basis for Small G Protein Effector Interaction of Rasrelated Protein 1 (Rap1) and Adaptor Protein Krev Interaction Trapped 1 (KRIT1). Journal of Biological Chemistry, 2012. 287(26): p. 22317-22327.

173. Kleaveland, B., et al., Regulation of cardiovascular development and integrity by the heart of glass-cerebral cavernous malformation protein pathway. Nat Med, 2009. 15(2): p. 169-76.

174. Glading, A., et al., KRIT-1/CCM1 is a Rapl effector that regulates endothelial cell-cell junctions. The Journal of Cell Biology, 2007. 179(2): p. 247-254.

175. Mitin, N.Y., et al., Identification and Characterization of Rain, a Novel Rasinteracting Protein with a Unique Subcellular Localization. Journal of Biological Chemistry, 2004. 279(21): p. 22353-22361.

176. Post, A., et al., Rasip1 mediates Rapl regulation of Rho in endothelial barrier function through ArhGAP29. Proc Natl Acad Sci U S A, 2013. 110(28): p. 1142732.

177. Post, A., et al., Rap1 Spatially Controls ArhGAP29 To Inhibit Rho Signaling during Endothelial Barrier Regulation. Molecular and Cellular Biology, 2015. 35(14): p. 2495-2502.

178. Whitehead, K.J., et al., The cerebral cavernous malformation signaling pathway promotes vascular integrity via Rho GTPases. Nat Med, 2009. 15(2): p. 177-184.

179. Davis, G.E., K.J. Bayless, and A. Mavila, Molecular basis of endothelial cell morphogenesis in three-dimensional extracellular matrices. The Anatomical Record, 2002. 268(3): p. 252-275.

180. Egginton, S. and M. Gerritsen, Lumen Formation In Vivo Versus In Vitro Observations. Microcirculation, 2003. 10(1): p. 45-61.

181. Wang, Y., et al., Moesin1 and Ve-cadherin are required in endothelial cells during in vivo tubulogenesis. Development, 2010. 137(18): p. 3119-28.

182. Iruela-Arispe, M.L. and G.J. Beitel, Tubulogenesis. Development, 2013. 140(14): p. 2851-2855. 
183. Sorkin, A., Cargo recognition during clathrin-mediated endocytosis: a team effort. Curr Opin Cell Biol, 2004. 16(4): p. 392-9.

184. Schmid, E.M. and H.T. McMahon, Integrating molecular and network biology to decode endocytosis. Nature, 2007. 448(7156): p. 883-8.

185. Kirkham, M. and R.G. Parton, Clathrin-independent endocytosis: new insights into caveolae and non-caveolar lipid raft carriers. Biochim Biophys Acta, 2005. 1745(3): p. 273-86.

186. Kirkham, M., et al., Ultrastructural identification of uncoated caveolinindependent early endocytic vehicles. J Cell Biol, 2005. 168(3): p. 465-76.

187. Mayor, S. and R.E. Pagano, Pathways of clathrin-independent endocytosis. Nat Rev Mol Cell Biol, 2007. 8(8): p. 603-612.

188. Doherty, G.J. and H.T. McMahon, Mechanisms of endocytosis. Annu Rev Biochem, 2009. 78: p. 857-902.

189. West, M.A., et al., Rac is required for constitutive macropinocytosis by dendritic cells but does not control its downregulation. Curr Biol, 2000. 10(14): p. 839-48.

190. Dharmawardhane, S., et al., Regulation of Macropinocytosis by $\mathrm{p} 21$-activated Kinase-1. Molecular Biology of the Cell, 2000. 11(10): p. 3341-3352.

191. Veithen, A., et al., $v$-Src induces constitutive macropinocytosis in rat fibroblasts. J Cell Sci, 1996. 109 ( Pt 8): p. 2005-12.

192. Lisanti, M.P., et al., Characterization of caveolin-rich membrane domains isolated from an endothelial-rich source: implications for human disease. J Cell Biol, 1994. 126(1): p. 111-26.

193. Griffoni, C., et al., Knockdown of caveolin-1 by antisense oligonucleotides impairs angiogenesis in vitro and in vivo. Biochem Biophys Res Commun, 2000. 276(2): p. 756-61.

194. Liu, J., et al., Caveolin-1 expression enhances endothelial capillary tubule formation. J Biol Chem, 2002. 277(12): p. 10661-8.

195. Frank, P.G., et al., Caveolin, Caveolae, and Endothelial Cell Function. Arteriosclerosis, Thrombosis, and Vascular Biology, 2003. 23(7): p. 1161-1168. 
196. Liu, J., et al., Organized endothelial cell surface signal transduction in caveolae distinct from glycosylphosphatidylinositol-anchored protein microdomains. J Biol Chem, 1997. 272(11): p. 7211-22.

197. Rizzo, V., et al., Rapid mechanotransduction in situ at the luminal cell surface of vascular endothelium and its caveolae. J Biol Chem, 1998. 273(41): p. 26323-9.

198. Chavrier, P., et al., Localization of low molecular weight GTP binding proteins to exocytic and endocytic compartments. Cell, 1990. 62(2): p. 317-29.

199. Zerial, M. and H. McBride, Rab proteins as membrane organizers. Nat Rev Mol Cell Biol, 2001. 2(2): p. 107-117.

200. Maxfield, F.R. and T.E. McGraw, Endocytic recycling. Nat Rev Mol Cell Biol, 2004. 5(2): p. 121-32.

201. Stenmark, H., Rab GTPases as coordinators of vesicle traffic. Nat Rev Mol Cell Biol, 2009. 10(8): p. 513-525.

202. Hutagalung, A.H. and P.J. Novick, Role of Rab GTPases in membrane traffic and cell physiology. Physiol Rev, 2011.91(1): p. 119-49.

203. Pereira-Leal, J.B. and M.C. Seabra, Evolution of the Rab family of small GTPbinding proteins. J Mol Biol, 2001. 313(4): p. 889-901.

204. Schwartz, S.L., et al., Rab GTPases at a glance. J Cell Sci, 2007. 120(Pt 22): p. 3905-10.

205. Tarbutton, E., et al., Class I FIPs, Rab11-binding proteins that regulate endocytic sorting and recycling. Methods Enzymol, 2005. 403: p. 512-25.

206. Ducharme, N.A., et al., MARK2/EMK1/Par-1Balpha phosphorylation of Rab11family interacting protein 2 is necessary for the timely establishment of polarity in Madin-Darby canine kidney cells. Mol Biol Cell, 2006. 17(8): p. 3625-37.

207. Desclozeaux, M., et al., Active Rab11 and functional recycling endosome are required for E-cadherin trafficking and lumen formation during epithelial morphogenesis. Vol. 295. 2008. C545-C556.

208. Das, A. and W. Guo, Rabs and the exocyst in ciliogenesis, tubulogenesis and beyond. Trends in Cell Biology, 2011. 21(7): p. 383-386. 
209. Roland, J.T., et al., Rab GTPase-Myo5B complexes control membrane recycling and epithelial polarization. Proceedings of the National Academy of Sciences, 2011. 108(7): p. 2789-2794.

210. Gálvez-Santisteban, M., et al., Synaptotagmin-like proteins control the formation of a single apical membrane domain in epithelial cells. Nat Cell Biol, 2012. 14(8): p. 838-849.

211. Kuroda, T.S., et al., The Slp homology domain of synaptotagmin-like proteins 1-4 and Slac2 functions as a novel Rab27A binding domain. J Biol Chem, 2002. 277(11): p. 9212-8.

212. Martens, S. and H.T. McMahon, Mechanisms of membrane fusion: disparate players and common principles. Nat Rev Mol Cell Biol, 2008. 9(7): p. 543-556.

213. Li, C., et al., Ca2+-dependent and-independent activities of neural and nonneural synaptotagmins. Nature, 1995. 375(6532): p. 594-599.

214. Tang, J., et al., A complexin/synaptotagmin 1 switch controls fast synaptic vesicle exocytosis. Cell, 2006. 126(6): p. 1175-87.

215. Sutton, R.B., et al., Crystal structure of a SNARE complex involved in synaptic exocytosis at 2.4 A resolution. Nature, 1998. 395(6700): p. 347-53.

216. Jahn, R. and R.H. Scheller, SNAREs--engines for membrane fusion. Nat Rev Mol Cell Biol, 2006. 7(9): p. 631-43.

217. Hannah, M.J., et al., Weibel-Palade bodies recruit Rab27 by a content-driven, maturation-dependent mechanism that is independent of cell type. J Cell Sci, 2003. 116(Pt 19): p. 3939-48.

218. Lowenstein, C.J., C.N. Morrell, and M. Yamakuchi, Regulation of Weibel-Palade Body Exocytosis. Trends in Cardiovascular Medicine, 2005. 15(8): p. 302-308.

219. Wagner, D.D., The Weibel-Palade body: the storage granule for von Willebrand factor and P-selectin. Thromb Haemost, 1993. 70(1): p. 105-10.

220. Bierings, R., et al., The interplay between the Rab27A effectors Slp4-a and MyRIP controls hormone-evoked Weibel-Palade body exocytosis. Blood, 2012. 120(13): p. 2757-67.

221. de Leeuw, H.P., et al., Small GTP-binding protein RalA associates with WeibelPalade bodies in endothelial cells. Thromb Haemost, 1999. 82(3): p. 1177-81. 
222. Rondaij, M.G., et al., Small GTP-binding protein Ral is involved in cAMPmediated release of von Willebrand factor from endothelial cells. Arterioscler Thromb Vasc Biol, 2004. 24(7): p. 1315-20.

223. Moskalenko, S., et al., The exocyst is a Ral effector complex. Nat Cell Biol, 2002. 4(1): p. 66-72.

224. Moskalenko, S., et al., Ral GTPases Regulate Exocyst Assembly through Dual Subunit Interactions. Journal of Biological Chemistry, 2003. 278(51): p. 5174351748 . 


\title{
CHAPTER II
}

\section{CDC42 AND K-RAS CONTROL ENDOTHELIAL TUBULOGENESIS AND APICAL POLARIZATION: NEGATIVE REGULATION BY ARHGAP31 AND RASA1}

\author{
Pieter R. Norden ${ }^{1}$, Dae Joong Kim ${ }^{1}$, David M. Barry ${ }^{2}$, \\ Ondine B. Cleaver ${ }^{2}$, and George E. Davis ${ }^{1}$
}

\footnotetext{
${ }^{1}$ Department of Medical Pharmacology and Physiology, University of Missouri School of Medicine, Dalton Cardiovascular Research Center, Columbia, MO 65212

${ }^{2}$ Department of Molecular Biology, UT Southwestern Medical Center, Dallas TX 75390
} 


\begin{abstract}
Here, we identify major co-regulators of endothelial cell (EC) lumen formation including Cdc42 and k-Ras, their downstream effectors, IQGAP1, MRCK $\beta, \beta$-Pix, and Rasip1, and their negative regulators, Arhgap31 and Rasa1. Multiprotein complexes containing these molecules as well as other key regulators including $\alpha 2 \beta 1$ integrin and MT1-MMP can be shown to assemble during these events. Human EC siRNA suppression or mouse knockout of Rasip1 leads to identical phenotypes where ECs form extensive cord networks, but cannot generate lumens or tubes. Essential roles for these molecules during EC tubulogenesis include; i) establishment of asymmetric EC cytoskeletal polarization (subapical distribution of acetylated tubulin and basal distribution of F-actin); and ii) directed membrane trafficking of pinocytic vacuoles or other intracellular vesicles to the developing apical membrane surface. Cdc42 colocalizes subapically with acetylated tubulin and Rac1 strongly labels vacuole/ vesicle membranes, which accumulate and fuse together in a polarized, pericentrosomal manner. Overall, we observe apical membrane and subapical accumulation of key GTPases and effectors regulating EC lumen formation including Cdc42, Rac1, Rac2, k-Ras, Rap1b, activated c-Raf and Rasip1 to control EC tube network assembly.
\end{abstract}




\section{INTRODUCTION}

In recent years, considerable progress has been made toward our understanding of vascular morphogenesis, including the subject of this manuscript, which addresses how endothelial cells form tube networks with defined lumens ${ }^{1-6}$. Previous work has shown the critical importance of integrins, membrane-type matrix metalloproteinases (MT1MMP), Rho GTPases, particularly Cdc42 and Rac1, small GTPase regulators such as Rasip1, kinase cascades involving PKCepsilon (PKCE), Src family members, Pak2, Pak4, Raf, Mek and Erk, and the cytoskeleton ${ }^{3-5,7-13}$. Other interesting EC lumen regulators are proteins such as the cerebral cavernous malformation (CCM) proteins, CCM1, CCM2, CCM2L, and CCM3, as well as the polarity proteins, Par6b, Par3 and junctional adhesion receptors with affinity for Par3 including JamB, JamC and VE-cadherin ${ }^{4,8,14-19}$. An important direction of this work is to understand how ECs become polarized during lumen formation ${ }^{20}$. The role of polarity regulators has been demonstrated (i.e. Cdc42, Par6b, Par3), but how this contributes to the development of an EC apical surface and polarized cytoskeletal apparatus remains unclear.

We recently demonstrated that EC lumen formation in 3D matrices results in part due to the establishment of asymmetric cytoskeletal polarization with F-actin expressed in a basal fashion and with modified tubulins including acetylated and detyrosinated tubulin localized in a subapical domain to support the developing apical membrane surface $^{21}$. Key plus-end microtubule regulatory proteins, EB1, p150 ${ }^{\text {glued }}$ and Clasp1, control EC lumen formation through the subapical polarization and expression levels of these modified tubulins ${ }^{21}$. In part they act together to negatively regulate the tubulin 
deacetylases, HDAC6 and Sirt2. siRNA suppression of these deacetylases singly or in combination, led to increased EC lumen formation, while increased expression of HDAC6 and Sirt2 interfered with lumen formation ${ }^{21}$. In addition, disruption of microtubules with colchicine or other agents such as the chemotherapeutic drug, vinblastine, caused EC tube disassembly and collapse and importantly, there is also rapid loss of tubulin acetylation and activation of RhoA. Thus, tubulin modifications are major regulators of EC lumen formation, but also lumen and tube maintenance via support of the apical membrane domain ${ }^{21,22}$.

Here, in this new study, we have characterized the role of other small GTPases, downstream effectors, and key GTPase activating proteins (GAPs) that negatively regulate the EC lumen and tube formation process. Our studies have identified important new roles for Cdc42, Rac2, k-Ras and Rap1b and siRNA suppression of Cdc42 in combination with these other three GTPases causes profound inhibition of EC tubulogenesis. Furthermore, we have identified Arhgap31 and Rasa1 as GAPs that interfere with $\mathrm{Cdc} 42$, Rac, and k-Ras, markedly blocking the lumen formation process. In contrast, Arhgap29, a Rho-specific GAP, does the opposite and actually stimulates EC tube assembly through its RhoA-inhibitory activity. Additionally, we identify a novel role for downstream effectors of these GTPases including IQGAP1, MRCK $\beta$, $\beta$-Pix, GIT1, and Rasip1 during this process. Finally, we investigate EC polarization during lumen formation and demonstrate the apical targeting of small GTPases through membrane trafficking events along acetylated tubulin-enriched microtubule tracks, as well as the apical membrane targeting of key downstream regulators including Rasip1 and c-Raf. 


\section{MATERIALS AND METHODS}

\section{Reagents}

Stem cell factor (SCF), stromal cell-derived factor 1 alpha (SDF-1 $\alpha$ ), and interleukin-3 (IL-3) were obtained from R\&D systems (Minneapolis, MN). Tubacin was obtained from TOCRIS Bioscience (Bristol, United Kingdom). Ascorbic acid, 12-Otetradecanoyl-phorbol-13-acetate (TPA), and antibodies against Arhgap31, acetylated tubulin, $\alpha$-tubulin, and phospho-C-Raf Tyr341 were obtained from Sigma-Aldrich (St. Louis, MO). Recombinant fibroblast growth factor 2 (FGF-2) and antibodies against Rac2, detyrosinated tubulin, and $\beta$-actin was obtained from EMD Millipore. Antibodies against MRCK $\beta$, k-Ras, Rasip1, GIT1, and MT1-MMP were obtained from Abcam (Cambridge, MA). Antibodies against IQGAP1, ROCK1, and Integrin $\alpha 2$ were obtained from BD Biosciences (San Jose, CA). Antibodies against Rap1B, $\alpha$-Pix, $\beta$-Pix, Cdc42, phospho-p44/42 MAPK (ERK 1/2) Thr202/Tyr204, ERK 1/2, phospho-PAK2 Ser141, PAK2, phospho-PAK4 Ser474, PAK4, phospho-B-Raf Thr401, phospho-B-Raf Ser445, B-Raf, phospho-C-Raf Ser388, C-Raf, phospho-Src Y416, Src, phospho-p38 MAPK Thr180/Tyr182, p38 MAPK, and phospho-Tyr were obtained from Cell Signaling Technologies (Danvers, MA). An antibody against Rasa1 was obtained from Epitomics (Burlingame, CA). Antibodies against RhoA and Rac1 were obtained from Cytoskeleton (Denver, CO). An antibody against Arhgap29 was from Bethyl Laboratories (Montgomery, TX). An antibody against GAPDH was purchased from Research Diagnostics Inc (Flanders, NJ). Alexa fluor ${ }^{\circledR} 488$ and Alexa fluor ${ }^{\circledR} 633$ antibodies, and Alexa fluor ${ }^{\circledR} 488$ and 633 phalloidin were from Molecular Probes (Eugene, OR). WT 
PKC $\varepsilon$ adenovirus was purchased from Seven Hills Bioreagents (Cincinnati, OH) and WT CSK and DN CSK adenoviruses were purchased from Cell Biolabs (San Diego, CA). GFP, GFP-Cdc42, GFP-Rac1, GFP-RhoA, GFP-N17Rac1, GFP-N19RhoA, GFPN17Cdc42 and GFP-V12Rac1 adenoviruses were generated as previously described ${ }^{7}$, as well as DN-Pak4 adenovirus ${ }^{8}$, and MT1- $\Delta \mathrm{C}$ WT and MT1- $\Delta \mathrm{C}$ EA adenoviruses ${ }^{10,14}$.

Vasculogenic tube assembly assays

Human umbilical vein endothelial cells were obtained from Lonza (Walkersville, MD) and were cultured (passage 3-6) as described previously ${ }^{23}$. ECs were then suspended at $2 \times 10^{6}$ cells $/ \mathrm{mL}$ in $2.5 \mathrm{mg} / \mathrm{mL}$ collagen type I matrices and assays were performed as previously described ${ }^{24,25}$. Briefly, SCF, IL-3, SDF-1 $\alpha$, and FGF-2 were added at $200 \mathrm{ng} / \mathrm{mL}$ into collagen type I. Cultures were fed with media containing reduced serum supplement (RSII), ascorbic acid, and FGF-2 at $40 \mathrm{ng} / \mathrm{mL}$. Cultures were allowed to assemble into capillary networks over a period of 0-120 $\mathrm{hr}$ when cultures were fixed or collected for further processing. Samples were fixed in 2\% paraformaldehyde or 3\% glutaraldehyde in PBS. Cultures fixed in paraformaldehyde were then stained for fluorescent microscopy imaging, whereas cultures fixed in glutaraldehyde were stained in $0.1 \%$ toluidine blue in $30 \%$ methanol. Additionally unfixed collagen gels were lysed to examine protein expression at the indicated time points using standard western blotting techniques. Recombinant adenovirus infection of ECs was performed as previously described ${ }^{7}$. 


\section{EC siRNA suppression}

siRNA suppression protocols using the siRNA list below were performed as

previously described ${ }^{23}$. The cells were allowed to recover for $48 \mathrm{hr}$ and transfection was repeated. The cells were then allowed to recover overnight before being harvested for use in 3D assays.

siRNAs from Ambion are as follows:

Control (AM4637) Silencer Select Negative Control \#2

Cdc42 (s2765) 5'-UGGUGCUGUUGGUAAAACA-3'

Rac1 (s11711) 5'-CUACUGUCUUUGACAAUUA-3'

$\underline{\operatorname{Rac} 2}$ (s11714) 5'-CCUCUUUUGGAACAACAUA-3'

RhoA (s758) 5'-CACAGUGUUUGAGAACUAU-3'

k-Ras (s7939) 5'-CUAUGGUCCUAGUAGGAAA-3'

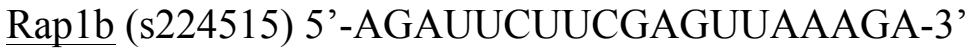

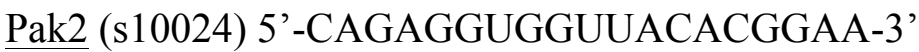

Rasip1 (s29763) 5'-CGAGCUGUUCAAAUCCGAA-3'

a-Pix (s16948) 5'-GUAAAAGCCCUAAAACGAU-3'

$\beta$-Pix (s18122) 5'-CAACGACAGGAAUGACAAU-3'

GIT1 (s26306) 5'-CCUUGAUCAUCGACAUUCU-3'

Rock1 (s12097) 5'-GGUUAGAACAAGAGGUAAA-3'

Arhgap31 (s33202) 5'-GGACAGAUCUCUACAUAGA-3'

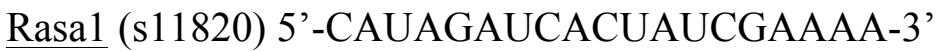

Arhgap29 (s485) 5'-GACCAAGGCUAAAACGAAU-3'

Stealth siRNAs from Invitrogen are as follows: 
Pak4 (NM_001014834_stealth_749)

5'-UGCUUGCGCAGGUCCAUCUUCUUGA-3'

IQGAP1 (NM_003870_stealth_421)

5'-GCCUCCACUUUAGACACACUGAUAA-3'

MRCK $\beta$ (NM_006035_stealth 691)

5'-UAAAUCACCACCCACAUAGUAAUCC-3'

Generation of S-epitope tagged Cherry, Cherry-fusion proteins, and AcGFP-Rasip1 adenoviruses

Cdc42, Rac1, Rac2, RhoA, k-Ras, and Rap1b were amplified from cDNA obtained from Missouri S\&T cDNA Resource Center (Rolla, MO) and human Rasip1 and PKC $\varepsilon$ were amplified from cDNA obtained from Open Biosystems (Open Biosystems, GE Dharmacon, Lafayette, CO), and standard restriction digest cloning for individual GTPases and PKCe into pmCherry-C1 plasmid and Rasip1 into pAcGFP-C1 (Clontech, Mountain View, CA) using EcoRI-HF and BamHI-HF, XhoI and BamHI-HF, and EcoRI$H F$ and $\mathrm{XbaI}$ restriction enzymes respectively (New England Biolabs). Amplified SCherry, S-Ch-GTPase, S-Ch-PKCe, and AcGFP-Rasip1 constructs were subcloned into pShuttle-CMV expression plasmid using NotI-HF, XbaI, XhoI, KpnI-HF and SalI-HF restriction enzymes, respectively (New England Biolabs). Recombinant adenoviral vectors were then generated ${ }^{26}$ and propagated as previously described ${ }^{7}$. The PCR primers used are listed below with the upstream first followed by the downstream primer. Cdc42 5'AGGAATTCTATGCAGACAATTAAGTGTGTTG-3' 5'-AGGGATCCTTAGAATATACAGCACTTCCTTTT-3' 
Rac1 5'-AGGAATTCTATGCAGGCCATCAAGTGTGTGGTG-3'

5'-AGGGATCCTTACAACAGCAGGCATTTTCTCTTC-3'

Rac2 5'-AGGAATTCTATGCAGGCCATCAAGTGTGTGGTG-3'

5'AGGGATCCCTAGAGGAGGCTGCAGGCGCGCTTC-3'

RhoA 5'-AGGAATTCTATGGCTGCCATCCGGAAGAAACTG-3'

5’-AGGGATCCTCACAAGACAAGGCACCCAG-3'

k-Ras 5'-AGCTCGAGCTATGACTGAATATAAACTTGTGGTAG-3'

5'-AGGGATCCTTACATAATTACACACTTTGTCTTTG-3'

Rap1b 5'-AGGAATTCTATGCGTGAGTATAAGCTAGTCG-3'

5'-AGGGATCCTTAAAGCAGCTGACATGATGAC-3'

Rasip1 5'-AGGAATTCTATGCTGTCTGGTGAACGGAAGGAGG-3'

5'-AGGTCGACTCAAGGAGACGTGGCCACGGGAGGCCCATG-3'

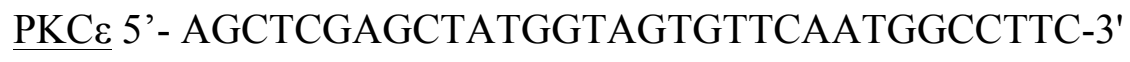

5'-AGGGATCCTCAGGGCATCAGGTCTTCACCAAAG-3'

Primers used for cloning into the pAdCMVShuttle plasmid are as follows. The first two primers (S-Cherry and AcGFP) are upstream primers while the following ones are downstream primers.

S-Cherry

5'-AGGCGGCCGCACCATGGCAAAAGAAACCGCTGCTGCGAAATTTG AACGCCAGCACATGGACTCGATGGTGAGCAAGGGCGAGGAG-3’

AcGFP 5'-AGGGTACCACCATGGTGAGCAAGGGCGCCGAGCTGTTCAC-3'

Cde42 5'-AGTCTAGATTAGAATATACAGCACTTCCTTTT-3'

Rac1 5'-AGTCTAGATTACAACAGCAGGCATTTTCTCTTC -3' 
Rac2 5'-AGTCTAGACTAGAGGAGGCTGCAGGCGCGCTTC-3'

RhoA 5'-AGTCTAGATCACAAGACAAGGCACCCAG-3'

k-Ras 5'-AGCTCGAGTTACATAATTACACACTTTGTCTTTG-3'

Rap1b 5'-AGTCTAGATTAAAGCAGCTGACATGATGAC-3'

PKCE 5'-AGTCTAGATCAGGGCATCAGGTCTTCACCAAAG-3'

Rasip1 5'-AGGTCGACTCAAGGAGACGTGGCCACGGGAGGCCCATG-3'

EC vasculogenesis pull-down assay

Pull down assays using S-epitope tagged mCherry GTPase fusion protein expressing adenoviruses were performed similarly to that previously described ${ }^{23}$. EC vasculogenesis assays were set up in $3.75 \mathrm{mg} / \mathrm{mL}$ collagen type I gels using adenovirus infected ECs and extracted at the indicated time points. 3D cultures were placed in lysis buffer consisting of $1 \%$ Triton X-100, $10 \mathrm{mM}$ Tris-base (pH 7.5), $150 \mathrm{mM} \mathrm{NaCl}, 1 \mathrm{mM}$ DTT and $5 \mathrm{mM} \mathrm{MgCl}_{2}$, or $1 \mathrm{mM} \mathrm{MgCl} 2$ and $1 \mathrm{mM} \mathrm{CaCl}_{2}$, Complete EDTA-free protease inhibitor cocktail tablets (Roche Diagnostics, Indianapolis, IN), collagenase $(150 \mu \mathrm{g} / \mu \mathrm{L}$ high-purity; Sigma-Aldrich), and $100 \mu \mathrm{M} \mathrm{GTP} \gamma \mathrm{S}$ (Calbiochem). Lysates were incubated in a $37^{\circ} \mathrm{C}$ water bath for 15 minutes to aid in collagen digestion and were clarified by centrifugation at $16,000 \mathrm{~g}$ for 20 minutes at $4^{\circ} \mathrm{C}$. Supernatants were then incubated with S-protein agarose beads (Novagen, EMD Millipore, Billerica, MA) equilibrated with washing buffer (respective lysis buffer containing $0.1 \%$ Triton $\mathrm{X}-100$ ) for 1 hour at $4^{\circ} \mathrm{C}$ on a rocking plate. Beads were then washed 4 times with washing buffer before bound protein was eluted with $1.5 \mathrm{X}$ sodium dodecyl sulfate sample buffer containing $7.5 \% \beta$ - 
Mercaptoethanol. Bound GTPase-associated proteins were detected by western blot analysis.

In vitro culture immunofluorescent staining, microscopic imaging, and analysis

For analysis of 3D cultures, immunostaining was carried out as previously described. Immunostained cultures were imaged using a confocal microscope (Leica TC5 SP5) connected to a multiphoton system (Leica, Buffalo Grove, IL) using excitation wavelengths of $488 \mathrm{~nm}$ and $543 \mathrm{~nm}$ or $488 \mathrm{~nm}$ and $633 \mathrm{~nm}$ sequentially. High-resolution images were captured using a $63 \mathrm{X}$ water immersion objective (NA 1.2) utilizing Leica Application Suite (LAS) software. Toluidine blue stained cultures were imaged using light microscopy on inverted microscopes (Eclipse TE2000-E; Nikon, Melville, NY with Photometrics CoolSNAPHQ2 camera, Tucson, AZ, and Olympus CKX41 with Olympus DP70 camera, Center Valley, PA). Photographs were analyzed using Metamorph software (Molecular Devices, Sunnyvale, CA) by tracing vessel area and lumen area. Time-lapse videomicroscopy was performed using light microscopy and a 20X objective with a fluorescent inverted microscope (DMI6000B, Leica) over a $72 \mathrm{hr}$ period.

Immunofluorescent and immunocytochemical staining of embryonic tissues

Female mice expressing the Rosa26 yellow fluorescent protein (YFP) reporter were mated with male mice expressing Cadherin5-CreERT2. Pregnant females were induced with tamoxifen (2mg/40g Mouse) at E12 and E13, and then embryos dissected at E14. Head dermis positive for YFP was isolated, fixed in $4 \%$ PFA/PBS for $1 \mathrm{hr}$ at $4^{\circ} \mathrm{C}$ then washed in PBS. Primary antibody incubations were carried out at $4^{\circ} \mathrm{C} \mathrm{O} / \mathrm{N}$ (diluted 1:300 for GFP, 1:100 for Rasip1), slides were washed in PBS, and then incubated in 
secondary antibody for 4hrs at RT (diluted 1:500). Slides were washed in PBS incubated with DAPI and mounted. Images were obtained using a LSM710 Meta Zeiss confocal. Antibodies used include: anti-GFP (Aves/ GFP-1020), Rasip1 (Novus Biologicals/ NB300-967), and Alexa Fluor 555 donkey anti-goat (Invitrogen/ A21432, Donkey antichicken 488 (Jackson Immuno Cat\#703-545-155). Rasip1 ${ }^{+/-}$and Rasip1 ${ }^{-/}$embryos were fixed in 4\% PFA/PBS and stored in 75\% ethanol. Embryos were processed and sectioned as described. Sections were incubated with primary antibody (diluted 1:100 PECAM, 1:100 Endomucin) overnight at $4^{\circ} \mathrm{C}$, and after washing, were incubated with Donkey antiRat HRP secondary antibody (diluted 1:100). The DAB reaction was performed using a peroxidase substrate kit (Vector). The slides were imaged using a NeoLumar stereomicroscope (Zeiss). Antibodies used include: Goat anti-rat IgG (Santa Cruz/ A10549), PECAM (BD Biociences/ 553370), Endomucin (Santa Cruz/ sc-65495).

$\underline{\text { Statistical analysis }}$

Statistical analysis was completed using Microsoft Excel. Statistical significance was set at minimum with $\mathrm{P}<0.05$. Student $\mathrm{t}$-tests were used when analyzing two groups within individual experiments (with a minimum $n=10$ ).

\section{Ethics Statement}

All animal studies were performed in accordance with UT Southwestern Medical Center Institutional Animal Care and Use Committee (IACUC) approved protocol APN 2008-0310, approval date September 25, 2014. Mice were euthanized by CO2 asphyxiation followed by cervical dislocation. 


\section{RESULTS}

Identification of new small GTPase regulators of EC tubulogenesis

Our laboratory has previously demonstrated a critical role for Cdc42 and Rac1, as well as key effectors such as Pak2, Pak4 and the polarity proteins, Par6b and Par3, during EC lumen formation ${ }^{8,14}$. We performed a broader small GTPase screen using siRNA suppression where we identified several new regulators as well as effectors of these GTPases (Figure 2.1 and see later on). We are now demonstrating a key role for k-Ras, Rac2 and Rap1b during this process (Figure 2.1, Supplemental Figure 2.1). In contrast, siRNA suppression of RhoA had no influence. We increased expression of these GTPases using wild-type proteins that were fused on their N-terminus with mCherry and an S-epitope tag (for biochemical pulldown assays). Increasing expression of Cdc42, kRas, and Raplb all significantly enhanced EC lumen formation, while others did not (Supplemental Figure 2.1). Using combinations of siRNAs, we demonstrated that knockdown of Cdc42 with k-Ras, Rac2, and Rap1b, appear to have the greatest blocking influence (Figure 2.1C,D) compared to Cdc42 knockdown alone. Combined knockdown of k-Ras with Rac1, Rac2 or Rap1b did not block in a significant manner compared to kRas alone (not shown). This data suggests that Cdc42-dependent signaling in combination with either k-Ras, Rac2 and Rap1b, appears to be necessary for ECs to form lumens and tubes in a 3D matrix environment.

Functionally interchangeable Factor- and Phorbol ester-induced human EC lumen and tube formation models 
Our studies have utilized two highly defined and related systems, which have allowed us to investigate the human EC lumen formation process. One of them utilizes defined growth factors (Factor-induced model) that drive EC tubulogenesis, while the other depends on the addition of phorbol ester ${ }^{23,24,27}$. Most of the studies in this report utilize the Factor-induced model ${ }^{24}$. To characterize this new system with regard to our previous published work using the phorbol ester model and EC lumen formation, we have performed detailed Western blots to assess signaling pathways and requirements during the tubulogenic process (Figure 2.2). We previously reported that the phorbol ester system required the protein kinase $\mathrm{C}$ (PKC) isoform, $\mathrm{PKC} \varepsilon$ and Src kinases as well as a downstream cascade leading to Pak2, Pak4, Raf and Erk activation in order to form EC lumens and tube networks ${ }^{9}$. Lumen formation in this system also requires the $\alpha 2 \beta 1$ integrin and membrane-type I metalloproteinase (MT1-MMP). Using the Factor model, lumen formation is markedly stimulated by $\mathrm{PKC} \varepsilon$, dominant negative Csk (to activate Src kinases) as well as a cytoplasmic tail deleted wild type MT1-MMP construct (Figure 2.2C), all of which mimic what we reported in past work ${ }^{9,14}$. Furthermore, we demonstrate that increased expression of Csk, to block Src activation, addition of the Src inhibitor, PP2 (but not PP3, its inactive control) (not shown) or expression of a dominant negative inhibitor of MT1-MMP (cytoplasmic tail deleted combined with an inactivating mutation) all dramatically interfere with lumen formation just like we previously observed (Figure 2.2C) ${ }^{9,14}$, suggesting that our two model systems appear to be functionally interchangeable. A final point is that we previously reported that increased tubulin acetylation and detyrosination accompany lumen formation and are necessary to stabilize the developing EC apical membrane using the phorbol ester model ${ }^{21}$. Here, we 
show the same increases in tubulin acetylation and detyrosination during lumen formation using the Factor system (Figure 2.2), and furthermore, addition of the HDAC6 inhibitor, tubacin, which strongly increases tubulin acetylation, leads to marked increases in lumen formation (Supplementary Figure 2.1).

Differential signaling events during Factor-induced EC lumen and tube formation

During the time course of lumen formation using the Factors, we observe increases in the expression of both $\alpha 2 \beta 1$ integrin and MT1-MMP, as well as increased phosphorylation of Src, Pak4, B-Raf, c-Raf, and Erk, while levels of p38 Map kinase phosphorylation remain low compared to controls (Figure 2.2). We also performed Western blots over time to assess whether changes in proteins that are tyrosine phosphorylated are differentially regulated during lumen formation. We observe increased tyrosine phosphorylation of bands during this process at 225, 150, 130, 120, $100,78,68,60,45,40,36$, and $30 \mathrm{kDa}$ (Figure 2.2). EC tubulogenesis is Src family- and receptor tyrosine kinase-dependent through Factor and extracellular matrix signaling events and work is ongoing to identify these tyrosine phosphorylated bands that are induced during this process.

Small GTPase targeting and polarization to apical membranes and the microtubule cytoskeleton enriched in acetylated tubulin during EC lumen formation

One of the key steps in lumen formation is creation of a unique apical surface. We are putting forth a considerable effort in our laboratory to define how the apical surface forms and stabilizes during EC lumen formation. For example, we recently 
showed that the modified tubulins, acetylated and detyrosinated tubulin are subapically polarized during lumen formation (Figure 2.3) and this depends on the microtubule plusend regulators, EB1, p150 $0^{\text {glued }}$ and Clasp $1^{21}$. Blockade of these molecules results in interference with lumen formation and apical polarization. By contrast, F-actin as visualized using phalloidin, is polarized in a basal location. Here, we have imaged the subcellular localization of small GTPases (using fluorescent fusion proteins) that directly influence this process. Cdc42, Rac1, Rac2, k-Ras, and Rap1b all show targeting ability to the apical surface in comparison to the basal distribution of F-actin (Figure 2.3, Supplementary Figure 2.1). In addition, a key activated effector downstream of these GTPases is phospho-c-Raf ${ }^{9,14}$, which also shows apical targeting during these events (Supplementary Figure 2.1).

Furthermore, we have examined the relationship between $\mathrm{Cdc} 42$ and Rac1 and the appearance of these proteins in the apical domain along with acetylated tubulin, which is strongly polarized subapically (Figure 2.3). Cdc42 concentrates in a subapical distribution that also shows focal co-localization with acetylated tubulin (Figure 2.3). By contrast, although acetylated tubulin is subapically distributed, GFP (control) or RhoA do not display apical localization. We also performed experiments by expressing constitutively active GFP-V12Rac1 (which enhances lumen formation) ${ }^{7}$, which stimulated the appearance of acetylated and detyrosinated tubulin and promoted localization of acetylated tubulin subapically (Figure 2.3, Supplementary Figure 2.2). Expression of dominant negative Cdc42, Rac1, and Pak4 constructs (which block lumen formation) ${ }^{7,8}$ reduced acetylated tubulin compared to control or dominant negative RhoA expression (Supplementary Figure 2.2). Using GFP-Rac1, GFP-V12Rac1, GFP- 
Cdc42 constructs versus control GFP or GFP-RhoA constructs, we are able to observe pinocytic intracellular vacuoles ${ }^{7,27}$ which traffic apically to fuse along modified tubulin cytoskeletal tracks in a subapical and pericentrosomal region to contribute to the EC apical luminal membrane over time (Figure 2.3).

Cdc42, Rac, and Ras GAPs, Arhgap31 and Rasa1, inhibit EC lumen formation, while RhoGAP, Arhgap29, stimulates this process

To address the role of specific GTPases in EC lumen formation using a distinct approach, we have identified three GTPase activating proteins (GAPs) (which inactivate the GTPases) that control the EC lumen formation process (Figure 2.4). Arhgap31 shows specificity for $\mathrm{Cdc} 42$ and $\mathrm{Rac}^{28}$, Rasa1 for Ras ${ }^{29}$, and Arhgap29 for RhoA ${ }^{11}$. Arhgap29 is a known binding partner of Rasip 1, a key regulator of EC lumen formation ${ }^{11}$. siRNA suppression of Arhhap31 and Rasa1 (or in combination) strongly stimulated EC lumen and tube formation, while suppression of Arhgap29 strongly inhibited (Figure 2.4). This latter result is consistent with previous observations from our laboratory where RhoA played a direct role in vessel collapse following microtubule disruption ${ }^{22}$. Increased expression of activated RhoA completely inhibited EC lumen formation ${ }^{7}$ and interestingly, infection of ECs with the microorganism, Bartonella bacilliformis, which led to RhoA activation, similarly blocked EC tube formation ${ }^{30}$.

To assess the influence of GAP knockdown on EC signaling during lumen formation, we performed Western blots at different time points during the process. This approach allows us to assess the relative contribution of Cdc42/Rac, k-Ras, and RhoA toward the signaling pathways that are activated during lumen formation (Figure 2.2, 
Figure 2.5). Interestingly, the expression of $\alpha 2 \beta 1$ integrin and MT1-MMP were modestly increased when Arhgap31 and Rasa1 are suppressed compared to control, and similar findings were observed with Pak2. Erk1/2 levels modestly increased selectively with Rasa1 knockdown that accompany stronger increases in Erk1/2 phosphorylation. We observe increased phosphorylation of Pak2 and Pak4 that occur following knockdown of either Arhgap31 or Rasa1 (or the combination). Since Pak4 is a selective effector of Cdc42, this result suggests that activation of k-Ras (via Rasa1 knockdown) leads to increased activation of $\mathrm{Cdc} 42$, demonstrating that these pathways are co-activating each other (perhaps through upstream guanine exchange factors- GEFs). Similar results were observed with Pak2 activation where Rasa1 knockdown increases Pak2 phosphorylation, an effector of both $\mathrm{Cdc} 42$ and Rac1/2. We also examined tyrosine phosphorylation of substrates in these samples and observed that bands at 150, 78, 60, 45, and $36 \mathrm{kDa}$ were increased when either Arhgap31 or Rasa1 (or both) were suppressed, while a band at 40 $\mathrm{kDa}$ appeared to be increased more selectively with Rasal knockdown. This data suggests that these phosphoproteins may be involved in the lumen formation process since their phosphorylation patterns correlate with EC tube formation ability (Figure 2.5) and they were also regulated during a time course of tube formation (Figure 2.2). Future work will attempt to identify these proteins and to determine their functions during these events.

Identification of new $\mathrm{Cdc} 42, \mathrm{Rac}$ and Ras effectors which control EC tubulogenesis

Previous work had identified key effectors of Cdc42 and Rac1-dependent EC lumen formation and they are, Pak2, Pak4, and Par6b ${ }^{8}$. Par6 binds Par3 which has 
affinity for the adhesion molecules, JamB, JamC, and VE-cadherin, which we and others have shown are critical to EC lumen formation and cell polarity during this process ${ }^{8,14}$ 16,19 . Here, we report the identity of new critical effectors in this lumen formation process and they are IQGAP1, MRCK $\beta$, Rasip1, GIT1 (a Pak2-binding protein), and $\beta$ Pix, a known Cdc42 and Rac effector, but also a GEF for these GTPases (Figure 2.6, Supplementary Figures 2.2, 2.3). No effects were observed from siRNA suppression of $\alpha$-Pix (which shows modest inhibitory activity) and Rock1, a known RhoA effector (Figure 2.6, Supplementary Figure 2.3).

To address if we could observe additive or synergistic effects of knockdown of multiple effectors, we performed combination experiments with more than one siRNA (Supplementary Figures 2.2,2.3). We also predicted that this type of analysis might facilitate our ability to delineate if these effectors were in the same or distinct signaling pathways affecting different steps in the EC lumen formation cascade. The greatest blocking combination of siRNAs is when ECs are treated with Pak2 and Pak4, suggesting their critical involvement and their likely participation in different steps of the process because of the strong additivity or possible synergism (Supplementary Figures 2.2, 2.3). Interestingly, both Pak2 and Pak4 siRNAs also additively block with siRNAs to MRCK $\beta$, Rasip1 and $\beta$-Pix, while Pak2 more selectively added to the blocking effects of IQGAP1 siRNA (Supplementary Figure 2.2). Additive blocking effects were observed with Rasip1 with Pak2, Pak4, IQGAP1 and MRCK $\beta$ siRNAs, but not with $\beta$-Pix, suggesting the possibility that they are functionally linked in a signaling pathway. Additive blocking effects were seen with MRCK $\beta$ combined with Pak2, Pak4, Rasip1, but not with either IQGAP1 or $\beta$-Pix. Finally, IQGAP1 siRNA showed additive blocking effects with Pak2 
and Rasip1, and $\beta$-Pix siRNA induced additive blocking effects when combined with either Pak2 or Pak4, but not the other effectors. A key point is that these molecules represent critical effectors of lumen formation which are directly linked to Cdc42-, Rac1, Rac2-, and k-Ras-dependent signaling and when we suppress the expression of these in combination (Supplementary Figure 2.2), there is a profound interference in the ability of ECs to form tubes.

EC tubulogenesis is controlled by Rasip1 in vitro and in vivo, and Rasip1 localizes to the $\underline{\text { EC apical membrane during lumen formation }}$

Previous work demonstrated a role for Rasip1 in EC lumen formation in vivo and in vitro ${ }^{11}$. Here, we extend these studies to show that using our new Factor system, Rasip1 siRNA treatment reveals a phenotype that directly recapitulates what we observe in vivo in the Rasip1 knockout animals (Figure 2.6). EC cords are observed to form and that reach their appropriate locations in the embryo, but they fail to lumenize (Figure 2.6C). We observe this exact phenotype in that they effectively assemble into cords in a pattern that resembles that of the control siRNA culture, but they fail to form lumens (Figure 2.6C). Thus, the in vivo knockout data is clearly reflected in the in vitro assays which show the same morphogenic phenotype. Our key findings using in vitro models of EC lumen formation and EC-pericyte tube co-assembly have repeatedly been demonstrated to be recapitulated in multiple species including Zebrafish, quail and mice.

To assess further a possible role for Rasip1 during these events, we constructed a vector carrying GFP-Rasip1 and transduced it into ECs and performed lumen formation assays. In addition, we increased PKC $\varepsilon$ expression which enhanced targeting of GFP- 
Rasip1 to the EC apical surface during lumen formation (Figure 2.6D). We also coexpressed GFP-Rasip1 with other mCherry control or GTPase fusion proteins and in all cases Rasip1 is observed to target apical surfaces during lumen formation over time (Figure 2.6E). In addition, immunofluorescence staining in vivo during mouse vascular development shows a similar distribution of Rasip1 which is observed more selectively along the EC apical surface during initiation of lumen opening (Figure 2.6D). This novel data suggests that Rasip1 can target the EC apical surface and this ability correlates with its ability to control lumen formation like the apical/subapical targeting of the key small GTPases (Figure 2.3, Supplementary Figure 2.1). Overall, our findings suggest that membrane transfer events from basal to apical are likely to underlie the molecular mechanisms that control the development and expansion of the EC apical membrane surface during lumen and tube formation.

Real-time video analysis reveals critical involvement of key GTPases, GAPs, and effectors during EC tubulogenesis

Another experimental approach that we have taken previously and also here is to perform real-time video analysis of EC tubulogenic responses of control vs. siRNA treated ECs. Control siRNA-treated cells form extensive networks of tubes over the 72 $\mathrm{hr}$ time period. During the first $12 \mathrm{hr}$ and later on also, vesicular trafficking and intracellular vacuole formation is observed which contributes to the lumen formation process (Video S1) and is demonstrated in our confocal imaging shown earlier (Figure 2.3). siRNA suppression of Cdc42 markedly interferes with EC tubulogenesis (Video S2). Interestingly, it appears that the ECs with reduced Cdc42 are attempting to form 
lumens and tubes, but then they collapse. In contrast, siRNA suppression of RhoA in ECs allows them to form lumens and tubes just like the control siRNA-treated ECs (Video S3). siRNA suppression of Arhgap31 (Video S4) and Rasa1 (Video S5) which activates Cdc42, Rac1 and k-Ras, respectively, leads to an acceleration of lumen and tube formation. In contrast, siRNA knockdown of Arhgap29, leading to activation of RhoA, leads to inhibition of EC tubulogenesis, where EC cord formation occurs, and attempts at tube formation are observed, but this is followed by collapse of the developing luminal space (Video S6). Very similar findings are observed with Rasip1 siRNA-treated ECs, where EC cord assembly occurs, but lumen formation does not (Video S7). Combined siRNA suppression of key lumen regulators including Cdc42 and k-Ras (Video S8), Cdc42 and Rac2 (Video S9), and Cdc42 and Rap1b (Video S10), reveal marked blockade of EC tubulogenesis.

Differential interactions of small GTPases with downstream effectors as well as MT1MMP and $\alpha 2 \beta 1$ integrin during EC lumen formation

To further address the functional biochemical connections of the GTPases and effectors in the EC lumen formation cascade, we performed pull-down assays using our S-Cherry GTPases constructs during this process in 3D matrices. We also stimulated lumen formation by increasing the expression of PKC $\varepsilon$ in order to assess if the pulldown assays correlate with the functional enhancement of lumen formation (Figure 2.7) (see Figure 2.2C). Consistently in our pulldown experiments, S-Ch-Rac2 and S-Ch-k-Ras strongly co-precipitate with MT1-MMP, but not S-Cherry control. These pulldowns were enhanced by increased expression of PKC $\varepsilon$. Similar results were observed with 
$\alpha 2 \beta 1$ pulldown and this is consistent with our previous observations demonstrating an interaction between MT1-MMP and $\alpha 2 \beta 1$ integrin ${ }^{14}$. This demonstrates that two key regulators of lumen formation, MT1-MMP and $\alpha 2 \beta 1$, also interact in multiprotein complexes with key small GTPases controlling lumen formation, namely Rac2 and kRas, and this is stimulated by increased PKC $\varepsilon$ expression. Lesser but detectable interactions were also observed with Cdc42 and Rac1, but not Cherry control. IQGAP1 strongly interacts with Cdc42 and also to a lesser extent with Rac1 (Figure 2.7A), but not the other proteins and these interactions increase as lumen formation proceeds (Figure 2.7B). MRCK $\beta$ interacts selectively with $\mathrm{Cdc} 42$ during the lumen formation process and this interaction is enhanced by increased PKC $\varepsilon$ expression (Figure 2.7A). Interestingly, MRCK $\beta$ is activated by diacylglycerol, like PKC $\varepsilon$. Finally, we performed these experiments and blotted for phosphotyrosine-containing proteins and identified unique interactions for the different GTPases (Figure 2.7). k-Ras interacts with two phosphotyrosine-containing proteins at 42 and $34 \mathrm{kDa}$, while Rac1 and Rac2 interact with a band at $40 \mathrm{kDa}$ and $\mathrm{Cdc} 42$ binds to a $45 \mathrm{kDa}$ band (Figure 2.7A). The identity of each of these bands will be pursued in future studies. When PKCe expression is enhanced, each of these bands increases their respective GTPase association. This data indicates unique associations of each GTPase during EC lumen formation. 


\section{DISCUSSION}

A critical question in vascular biology is how ECs assemble networks of tubes

with defined lumens ${ }^{2-5}$. A primary function of ECs is to undergo tubulogenesis, maintain tube structures, and then specialize into ECs with unique functions tailored to meet specific tissue requirements. Here, we focused on the molecular mechanisms underlying EC lumen and tube assembly. We identify new GTPase regulators of EC lumen formation as well as downstream regulators of these GTPases. In addition to reaffirming a key role for Cdc42, Rac1 and their effectors, Pak2 and Pak4, we demonstrate novel roles for Rac2, k-Ras, Rap1b, and the effectors, IQGAP1, MRCK $\beta, \beta$-Pix, GIT1 and Rasip1. Furthermore, we demonstrate important new roles for three GAPs: Arhgap31 which inactivates Cdc42 and Rac, Rasa1 which inactivates k-Ras, and Arhgap29 which inactivates RhoA. Arhgap31 and Rasa1 siRNAs were shown individually and in combination to stimulate tube formation (via blockade of Cdc42, Rac, and Ras respectively), while Arhgap29 siRNA inhibits tube formation (via blockade of RhoA). The above molecules appear to control EC tubulogenesis by affecting asymmetric cytoskeletal polarization (i.e. modified tubulins expressed subapically, F-actin basally), which is necessary to direct membrane trafficking events toward the new apical surface to create a defined lumen in a $3 \mathrm{D}$ environment. Furthermore, we demonstrate that Cdc42, Rac, k-Ras, Rasip1, and phospho-c-Raf accumulate at the developing apical membrane, while acetylated tubulin is strongly localized subapically to support this apical surface. Interestingly, in focal regions within this subapical domain, there is strong co-localization of Cdc42 with acetylated tubulin. 
A key conclusion of our findings is that small GTPase-mediated signaling in conjunction with a kinase signaling cascade involving PKCe, Src, Pak, Raf and Erk kinases leads to three major events that control EC tubulogenesis (Figure 2.8). The first is the establishment of asymmetric cytoskeletal polarity with subapical modified tubulins (particularly acetylated tubulin, but also detyrosinated tubulin), and F-actin distributed in a strong basal location. Our data suggests that this is necessary to direct membrane trafficking along subapically polarized microtubule tracks, which appears to facilitate focal apical accumulation of vesicles/vacuoles and subsequent vesicle fusion events to create the luminal space. The membranes that become the apical membrane surface are clearly enriched in small GTPases that affect EC lumen and tube formation including those that we evaluate here: $\mathrm{Cdc} 42$, Rac1, Rac2, k-Ras, and Rap1b. The second process is the creation of a polarized apical membrane surface. Vesicle/vacuole trafficking to this apical membrane appears to occur along the microtubule cytoskeleton and they accumulate in a pericentrosomal and perinuclear region. Thirdly, vesicle-to-vesicle fusion events (from vesicles derived from pinocytosed/ endocytosed membranes or other intracellular membranes derived from endoplasmic reticulum, Golgi or Weibel-Palade bodies) occur to generate an apical membrane template, which can then be further remodeled over time through endocytic and exocytic membrane trafficking events to develop a mature EC apical membrane domain. The molecular composition of this EC apical membrane domain is not well understood and ongoing work is addressing how it is assembled and maintained. Published real-time videos from our laboratory have revealed the dynamic nature of these membrane trafficking and vesicle fusion events which occur 
rapidly over a 4-72 $\mathrm{hr}$ period to create networks of capillary tubes with defined lumens ${ }^{2}$, $10,11,31,32$

Our new work demonstrates that multiple small GTPases contribute to EC tube formation, and we chose to focus our attention on $\mathrm{Cdc} 42$, Rac1, Rac2, k-Ras and Rap1b. siRNA suppression of Cdc42 in combination with k-Ras, Rac2 and Rap1b resulted in the most profound blocking effects, while blockade of Pak2 (activated by both Cdc42 and Rac isoforms) combined with multiple other effectors resulted in similar very strong blocking effects. Similar marked blocking effects were observed with Rasip1 siRNA suppression combined with either IQGAP1 or MRCK $\beta$, which are Ras, Rac and Cdc42 effectors ${ }^{33-36}$. This is highly supportive again of the conclusion that multiple GTPases and effectors work in concert to control this process. This is also demonstrated by siRNA suppression of Arhgap31 and Rasa1 together (to stimulate Cdc42, Rac and Ras activity in combination), where marked stimulation of EC tube formation occurs. Interestingly, other work supports our general conclusions, in that IQGAP1 is known to directly interact with k-Ras, b-Raf, and Erk ${ }^{33,37,38}$; thus serving as a scaffolding protein to promote Erk activity downstream of $\mathrm{Cdc} 42$ and Rac activation. In addition, $\mathrm{Cdc} 42$ and MRCK $\beta$ have been implicated in nuclear positioning in conjunction with centrosome reorientation during cell motility events ${ }^{39}$, and this process may also be critical during EC lumen formation since we observe accumulation of vesicles/vacuoles in a polarized pericentrosomal/perinuclear region. Src activity has been reported to inhibit the Rho/Rock pathway ${ }^{40}$, a pathway also inhibited by Pak2/Pak4 ${ }^{41}$ and Rasip1/Arhgap29 ${ }^{11}$. Interestingly, Rap1 is known to affect the function of Rasip1/Arhgap29 ${ }^{42}$. In addition, Src activation blocks the activity of Rasa ${ }^{43}$, while stimulating Pak2/Pak4 activation and 
Raf activation ${ }^{9}$. Interestingly, the Cdc42 guanine exchange factor (GEF), intersectin1, has been reported to inhibit the activity of Arhgap $31^{44}$. Future work in our laboratory will focus on the identification of the relevant GEFs which activate the key small GTPases that we have identified to control EC lumen and tube formation.

A major regulator of $\mathrm{EC}$ tubulogenesis is membrane trafficking from basal to apical, but possibly also from other intracellular membranes to the apical surface. In one of our first studies we demonstrated the presence of von Willebrand factor present inside intracellular vacuoles suggesting potential fusion of Weibel-Palade bodies with these vacuoles ${ }^{27}$. We observe the presence of intracellular vacuoles which are macropinosome-like structures (the vacuoles are strongly labeled by including membrane impermeant fluorescent dyes into the culture media showing that they are pinocytic) that are transported toward the apical surface to fuse with the luminal membrane ${ }^{27}$. These vacuole membranes can clearly be shown to possess Rac1 (very strongly observed when constitutively active Rac is utilized) suggesting that active Rac targets these membranes. Cdc42 appears to surround these structures in vitro and in vivo (when GFP-Cdc42 was expressed in ECs in Zebrafish embryos) ${ }^{7,31,45}$ and can be observed to accumulate subapically along with acetylated tubulin where focal areas of strong co-localization can be seen. These are also regions of probable vacuole-vacuole and vacuole-apical membrane fusion events that control the creation of the polarized luminal surface. Our new work suggests that Rasip1 can target apically and this is observed to a greater extent when we increase the expression of $\mathrm{PKC} \varepsilon$, a stimulus that markedly accelerates $\mathrm{EC}$ lumen and tube formation. Past work suggests that Ras can interact with Rasip1, so there may be a direct relationship with these findings, more studies will need to investigate 
these potential connections. Also, further work will need to address the role of different membrane compartments (basal to apical transfer through intracellular vacuoles) or other intracellular membranes (vesicle trafficking from endoplasmic reticulum or Golgi, or trafficking from structures such as Weibel-Palade bodies), and determine how individual proteins such as Rasip1 and Rac1 target to the apical surface.

One of the intriguing questions in cell biology is what are the necessary molecules and signals that are required for cells to generate lumens and tubes and what distinguishes them from cells that cannot. ECs and many types of epithelial cells can form tube structures, while cell types such as fibroblasts, pericytes, and vascular smooth muscle cells cannot. Of great interest is that we have identified a key series of molecules and signals that are necessary for ECs to form lumens and tubes. Genetic or chemical blockade of these molecules and signals converts ECs into cells that lose this ability to form lumens. We also presented data showing inhibitory roles for the GAPs Rasa1 and Arhgap31, and the lumen formation signaling cascade may function in part to suppresses their activity. Furthermore, previous work has suggested that EC lumen signaling suppresses RhoA- and Rock-dependent signaling and key EC molecules such as Rasip1, Arhgap29, CCM proteins, Src, Pak2 and Pak4 are known to suppress Rho/Rock activation $^{3,4}$. In addition, our previous work has identified other inhibitors of EC lumen formation including HDAC6, Sirt2, Csk, TIMP2 and TIMP3 $3^{9,21,32}$. It is becoming increasing clear that EC tube formation is controlled by a balance of stimulatory and inhibitory molecules (Figure 2.8). Vessel abnormalities could result from imbalances of signals from either one of these sets of molecules. How such a balance controls the ability of ECs to form tubes and other cells such as fibroblasts to not form tubes is a key 
cell biological problem and question that needs to be investigated in detail in future studies.

In addition to our efforts to elucidate how ECs form lumens and tubes in 3D matrices, we have previously investigated another key way in which ECs within capillaries become polarized and that is the selective recruitment of pericytes to the EC abluminal surface ${ }^{20,46,47}$. Of great interest, pericyte recruitment to EC-lined tubes in capillaries leads to another critical event which is the abluminal and, thus, polarized deposition of the vascular basement membrane, a process that requires both cell types ${ }^{46-}$ 48. An important question for future studies is how the tubulogenic regulators that we describe impact the ability of EC cord and tube networks to attract pericytes and induce vascular basement membrane assembly. Thus, the tubulogenic mechanism that we describe here is an essential and fundamental step in EC polarity and vessel maturation, which is further amplified by the recruitment and retention of pericytes around capillary tubes. 


\section{ACKNOWLEDGEMENTS}

The authors would like to thank Matthew Davis and Katherine Speichinger for help with the performance and analysis of experiments. 


\section{REFERENCES}

1. Adams RH, Alitalo K (2007) Molecular regulation of angiogenesis and lymphangiogenesis. Nat Rev Mol Cell Biol 8: 464-478.

2. Iruela-Arispe ML, Davis GE (2009) Cellular and molecular mechanisms of vascular lumen formation. Dev Cell 16: 222-231.

3. Davis GE, Stratman AN, Sacharidou A, Koh W (2011) Molecular basis for endothelial lumen formation and tubulogenesis during vasculogenesis and angiogenic sprouting. Int Rev Cell Mol Biol 288: 101-165.

4. Sacharidou A, Stratman AN, Davis GE (2012) Molecular mechanisms controlling vascular lumen formation in three-dimensional extracellular matrices. Cells Tissues Organs 195: 122-143.

5. $\quad \mathrm{Xu} \mathrm{K,} \mathrm{Cleaver} \mathrm{O} \mathrm{(2011)} \mathrm{Tubulogenesis} \mathrm{during} \mathrm{blood} \mathrm{vessel} \mathrm{formation.} \mathrm{Semin} \mathrm{Cell}$ Dev Biol 22: 993-1004.

6. Sigurbjornsdottir S, Mathew R, Leptin M (2014) Molecular mechanisms of de novo lumen formation. Nat Rev Mol Cell Biol 15: 665-676.

7. Bayless KJ, Davis GE (2002) The Cdc42 and Rac1 GTPases are required for capillary lumen formation in three-dimensional extracellular matrices. J Cell Sci 115: 1123-1136.

8. Koh W, Mahan RD, Davis GE (2008) Cdc42- and Rac1-mediated endothelial lumen formation requires Pak2, Pak4 and Par3, and PKC-dependent signaling. J Cell Sci 121: 989-1001.

9. Koh W, Sachidanandam K, Stratman AN, Sacharidou A, Mayo AM, et al. (2009) Formation of endothelial lumens requires a coordinated PKCepsilon-, Src-, Pakand Raf-kinase-dependent signaling cascade downstream of Cdc42 activation. J Cell Sci 122: 1812-1822.

10. Stratman AN, Saunders WB, Sacharidou A, Koh W, Fisher KE, et al. (2009) Endothelial cell lumen and vascular guidance tunnel formation requires MT1MMP-dependent proteolysis in 3-dimensional collagen matrices. Blood 114: 237 247. 
11. Xu K, Sacharidou A, Fu S, Chong DC, Skaug B, et al. (2011) Blood vessel tubulogenesis requires Rasip1 regulation of GTPase signaling. Dev Cell 20: 526539.

12. Barry DM, Xu K, Meadows SM, Zheng Y, Norden PR, et al. (2015) Cdc42 is required for cytoskeletal support of endothelial cell adhesion during blood vessel formation in mice. Development 142: 3058-3070.

13. Hoang MV, Nagy JA, Senger DR (2011) Cdc42-mediated inhibition of GSK3 beta improves angio-architecture and lumen formation during VEGF-driven pathological angiogenesis. Microvasc Res 81: 34-43.

14. Sacharidou A, Koh W, Stratman AN, Mayo AM, Fisher KE, et al. (2010) Endothelial lumen signaling complexes control 3D matrix-specific tubulogenesis through interdependent Cdc42- and MT1-MMP-mediated events. Blood 115: 5259-5269.

15. Whitehead KJ, Chan AC, Navankasattusas S, Koh W, London NR, et al. (2009) The cerebral cavernous malformation signaling pathway promotes vascular integrity via Rho GTPases. Nat Med 15: 177-184.

16. Lampugnani MG, Orsenigo F, Rudini N, Maddaluno L, Boulday G, et al. (2010) CCM1 regulates vascular-lumen organization by inducing endothelial polarity. $\mathrm{J}$ Cell Sci 123: 1073-1080.

17. Kleaveland B, Zheng X, Liu JJ, Blum Y, Tung JJ, et al. (2009) Regulation of cardiovascular development and integrity by the heart of glass-cerebral cavernous malformation protein pathway. Nat Med 15: 169-176.

18. Zheng X, Xu C, Smith AO, Stratman AN, Zou Z, et al. (2012) Dynamic regulation of the cerebral cavernous malformation pathway controls vascular stability and growth. Dev Cell 23: 342-355.

19. Zovein AC, Alfonso Luque A, Turlo KA, Hofmann JJ, Yee KM, et al. (2010) bl Integrin Establishes Endothelial Cell Polarity and Arteriolar Lumen Formation via a Par3-Dependent Mechanism. Dev Cell 18: 39-51.

20. Davis GE, Speichinger KR, Norden PR, Kim DJ, Bowers SLK (2015) Endothelial cell polarization during lumen formation, tubulogenesis and vessel maturation in $3 D$ extracellular matrices. In K Ebnet (Ed) "Cell Polarity I" Springer, New York: pg. 205-220. 
21. Kim DJ, Martinez-Lemus LA, Davis GE (2013) EB1, p150Glued, and Clasp1 control endothelial tubulogenesis through microtubule assembly, acetylation, and apical polarization. Blood 121: 3521-3530.

22. Bayless KJ, Davis GE (2004) Microtubule depolymerization rapidly collapses capillary tube networks in vitro and angiogenic vessels in vivo through the small GTPase Rho. J. Biol Chem 279: 11686-11695.

23. Koh W, Stratman AN, Sacharidou A, Davis GE (2008) In vitro three dimensional collagen matrix models of endothelial lumen formation during vasculogenesis and angiogenesis. Methods Enzymol 443: 83-101.

24. Stratman AN, Davis MJ, Davis GE (2011) VEGF and FGF prime vascular tube morphogenesis and sprouting directed by hematopoietic stem cell cytokines. Blood 117: 3709-3719.

25. Bowers SL, Meng CX, Davis MT, Davis GE (2015) Investigating human vascular tube morphogenesis and maturation using endothelial cell-pericyte co-cultures and a doxycycline-inducible genetic system in $3 D$ extracellular matrices. Methods Mol Biol 1189: 171-189.

26. He TC, Zhou S, da Costa LT, Yu J, Kinzler KW, et al. (1998) A simplified system for generating recombinant adenoviruses. Proc Natl Acad Sci U S A 95: 25092514.

27. Davis GE, Camarillo CW (1996) An alpha 2 beta 1 integrin-dependent pinocytic mechanism involving intracellular vacuole formation and coalescence regulates capillary lumen and tube formation in three-dimensional collagen matrix. Exp Cell Res 224: 39-51.

28. Lamarche-Vane N, Hall A (1998) CdGAP, a novel proline-rich GTPaseactivating protein for Cdc42 and Rac. J Biol Chem 273: 29172-29177.

29. Boon LM, Mulliken JB, Vikkula M (2005) RASA1: variable phenotype with capillary and arteriovenous malformations. Curr Opin Genet Dev 15: 265-269.

30. Verma A, Davis GE, Ihler GM (2001) Formation of stress fibres in human endothelial cells infected with Bartonella bacilliformis is associated with altered morphology, impaired migration and defects in cell morphogenesis. Cell Microbiol 3: 169-180.

31. Kamei M, Saunders WB, Bayless KJ, Dye L, Davis GE, et al. (2006) Endothelial tubes assemble from intracellular vacuoles in vivo. Nature 442: 453-456. 
32. Saunders WB, Bohnsack BL, Faske JB, Anthis NJ, Bayless KJ, et al. (2006) Coregulation of vascular tube stabilization by endothelial cell TIMP-2 and pericyte TIMP-3. J Cell Biol 175: 179-191.

33. White CD, Erdemir HH, Sacks DB (2012) IQGAPI and its binding proteins control diverse biological functions. Cell Signal 24: 826-834.

34. Smith JM, Hedman AC, Sacks DB (2015) IQGAPs choreograph cellular signaling from the membrane to the nucleus. Trends Cell Biol 25: 171-184.

35. Zhao Z, Manser E (2015) Myotonic dystrophy kinase-related Cdc42-binding kinases (MRCK), the ROCK-like effectors of Cdc42 and Rac1. Small GTPases: 18.

36. Mitin NY, Ramocki MB, Zullo AJ, Der CJ, Konieczny SF, et al. (2004) Identification and characterization of rain, a novel Ras-interacting protein with a unique subcellular localization. J Biol Chem 279: 22353-22361.

37. Matsunaga $\mathrm{H}$, Kubota $\mathrm{K}$, Inoue $\mathrm{T}$, Isono F, Ando O (2014) IQGAPI selectively interacts with $\mathrm{K}$-Ras but not with $\mathrm{H}$-Ras and modulates $\mathrm{K}$-Ras function. Biochem Biophys Res Commun 444: 360-364.

38. Ren JG, Li Z, Sacks DB (2007) IQGAPI modulates activation of B-Raf. Proc Natl Acad Sci U S A 104: 10465-10469.

39. Gomes ER, Jani S, Gundersen GG (2005) Nuclear movement regulated by Cdc42, MRCK, myosin, and actin flow establishes MTOC polarization in migrating cells. Cell 121: 451-463.

40. Im E, Kazlauskas A (2007) Src family kinases promote vessel stability by antagonizing the Rho/ROCK pathway. J Biol Chem 282: 29122-29129.

41. Callow MG, Zozulya S, Gishizky ML, Jallal B, Smeal T (2005) PAK4 mediates morphological changes through the regulation of GEF-H1. J Cell Sci 118: 18611872.

42. Post A, Pannekoek WJ, Ponsioen B, Vliem MJ, Bos JL (2015) Rapl spatially controls ArhGAP29 to inhibit Rho signaling during endothelial barrier regulation. Mol Cell Biol 35: 2495-2502.

43. Giglione C, Gonfloni S, Parmeggiani A (2001) Differential actions of p60c-Src and Lck kinases on the Ras regulators p120-GAP and GDP/GTP exchange factor CDC25Mm. Eur J Biochem 268: 3275-3283. 
44. Jenna S, Hussain NK, Danek EI, Triki I, Wasiak S, et al. (2002) The activity of the GTPase-activating protein CdGAP is regulated by the endocytic protein intersectin. J Biol Chem 277: 6366-6373.

45. Davis GE, Koh W, Stratman AN (2007) Mechanisms controlling human endothelial lumen formation and tube assembly in three-dimensional extracellular matrices. Birth Defects Res C Embryo Today 81: 270-285.

46. Stratman AN, Malotte KM, Mahan RD, Davis MJ, Davis GE (2009) Pericyte recruitment during vasculogenic tube assembly stimulates endothelial basement membrane matrix formation. Blood 114: 5091-5101.

47. Davis GE, Norden PR, Bowers SL (2015) Molecular control of capillary morphogenesis and maturation by recognition and remodeling of the extracellular matrix: functional roles of endothelial cells and pericytes in health and disease. Connect Tissue Res: 1-11.

48. Stratman AN, Davis GE (2012) Endothelial cell-pericyte interactions stimulate basement membrane matrix assembly: influence on vascular tube remodeling, maturation, and stabilization. Microsc Microanal 18: 68-80. 


\section{FIGURES AND LEGENDS}

\section{FIGURE 2.1}

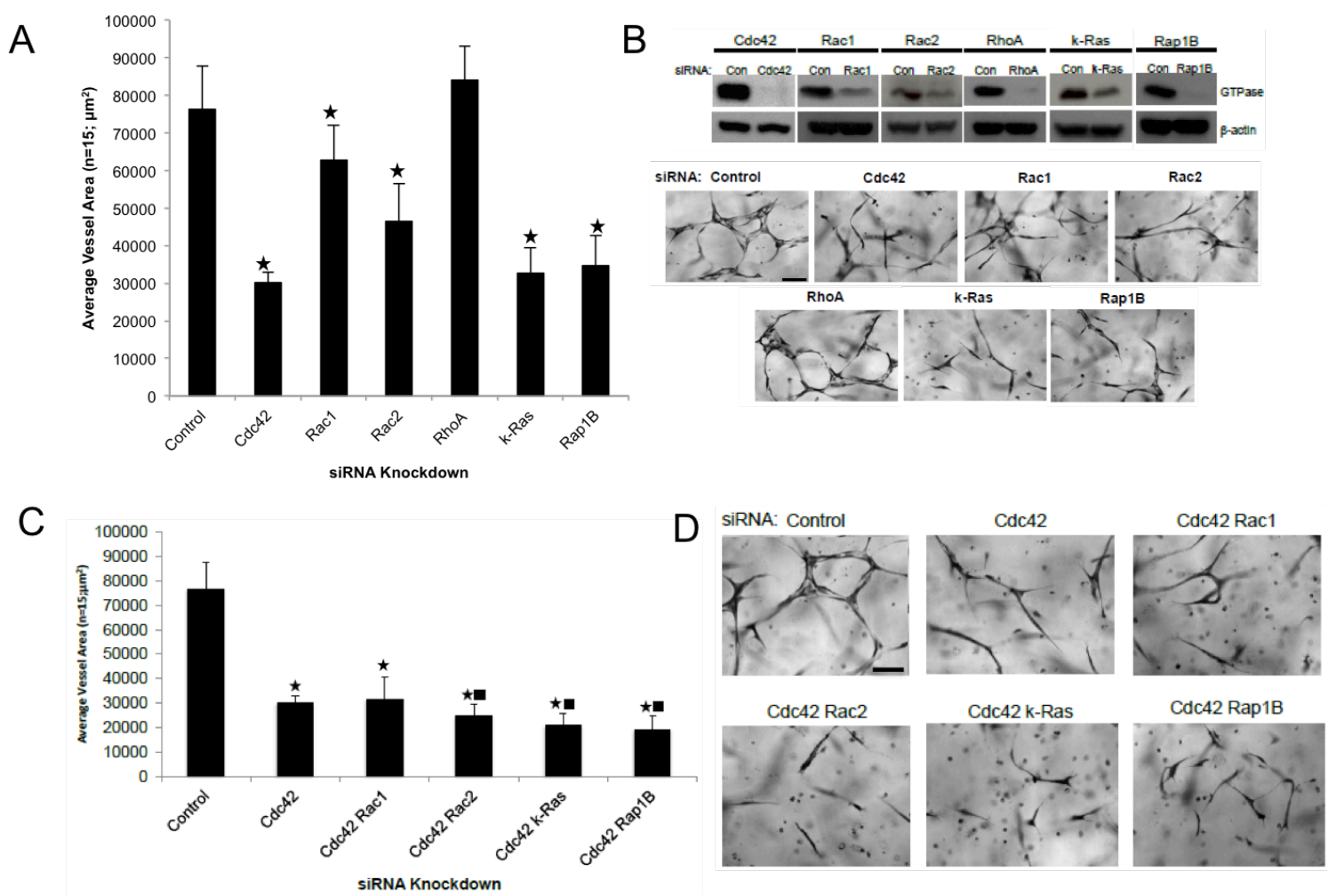

Figure 2.1. Identification of key small GTPases that control EC tubulogenesis in 3D matrices. (A) Individual EC cultures were transfected with control siRNA or with siRNAs directed to Cdc42, Rac1, Rac2, RhoA, k-Ras, and Rap1b, and then were suspended in 3D collagen matrices for $72 \mathrm{hr}$. Data are reported as the mean total vessel area per high-power field (HPF) \pm standard deviation (SD) $(n=15, \mathrm{p}<0.01)$. Asterisk indicates significance compared to control cultures. Fixed cultures were fixed, stained and photographed. Bar equals $100 \mu \mathrm{m}$ (B). (B) Lysates generated from siRNA transfected cultures in (A) were used in Western blots to assess specific protein knockdown versus control. (C,D) Individual EC cultures were transfected with siRNA targeting Cdc42, combinations of Cdc42 with Rac1, Rac2, k-Ras, and Rap1B siRNAs, or a control siRNA. EC tubulogenesis assays were performed with the cells and after $72 \mathrm{hr}$, cultures were fixed, photographed (D) and quantitated (C). Data are reported as the mean vessel area $\pm \mathrm{SD}(\mathrm{n}=15 ; \mathrm{p}<0.01)$. Asterisk indicates significance compared to control while the square indicates significance compared to Cdc42 siRNA treatment. Bar equals $100 \mu \mathrm{m}$. 


\section{FIGURE 2.2}
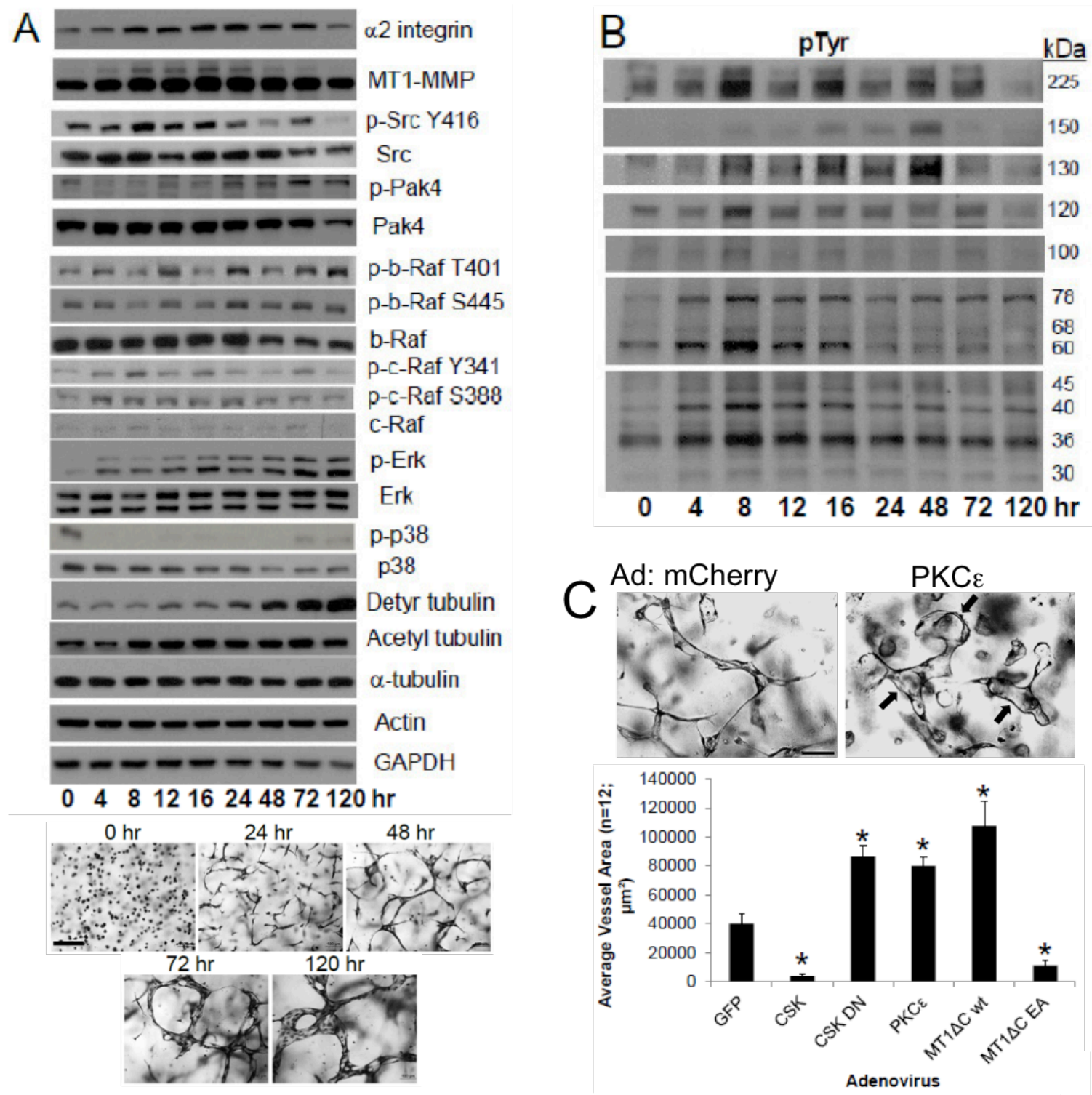

Figure 2.2. Signaling events that characterize EC tubulogenesis over time in $3 D$ collagen matrices. (A) ECs suspended in 3D collagen matrices were lysed at the indicated time points for Western Blot analysis to assess expression and signaling of the indicated molecules or were fixed and photographed while ECs are assembling into tubes over time. Representative blots are shown of 1 of 2 experiments. Bar equals $100 \mu \mathrm{m}$. (B) The same time courses were analyzed for phosphotyrosine (pTyr)-containing proteins and increased levels of pTyr bands at 225, 150, 130, 120, 100, 76, 68, 60, 45, 40, 36, and 30 $\mathrm{kDa}$ were identified over time correlating with the EC tubulogenic process. (C) ECs were infected with the indicated recombinant adenoviruses and then a lumen assay was performed over $72 \mathrm{hr}$. Upper panel- ECs were infected with mCherry or PKCe 
adenoviruses, then cultured in 3D collagen gels, fixed and photographed. Bar equals 100 $\mu \mathrm{m}$. Lower panel- ECs were infected with adenoviruses carrying GFP, Csk, DN Csk, PKC $\varepsilon$, tail-deleted catalytically active WT MT1-MMP (MT1- $\Delta$ CT wt) or a dominant negative MT1-MMP construct (MT1- $\triangle$ CT EA), then suspended in 3D collagen matrices to assess their ability to form lumens. Data are reported as the mean vessel area $\pm \mathrm{SD}$ per HPF $(n=12 ; \mathrm{p}<0.01)$. Asterisks indicate significance compared to control GFP cultures.

\section{FIGURE 2.3}
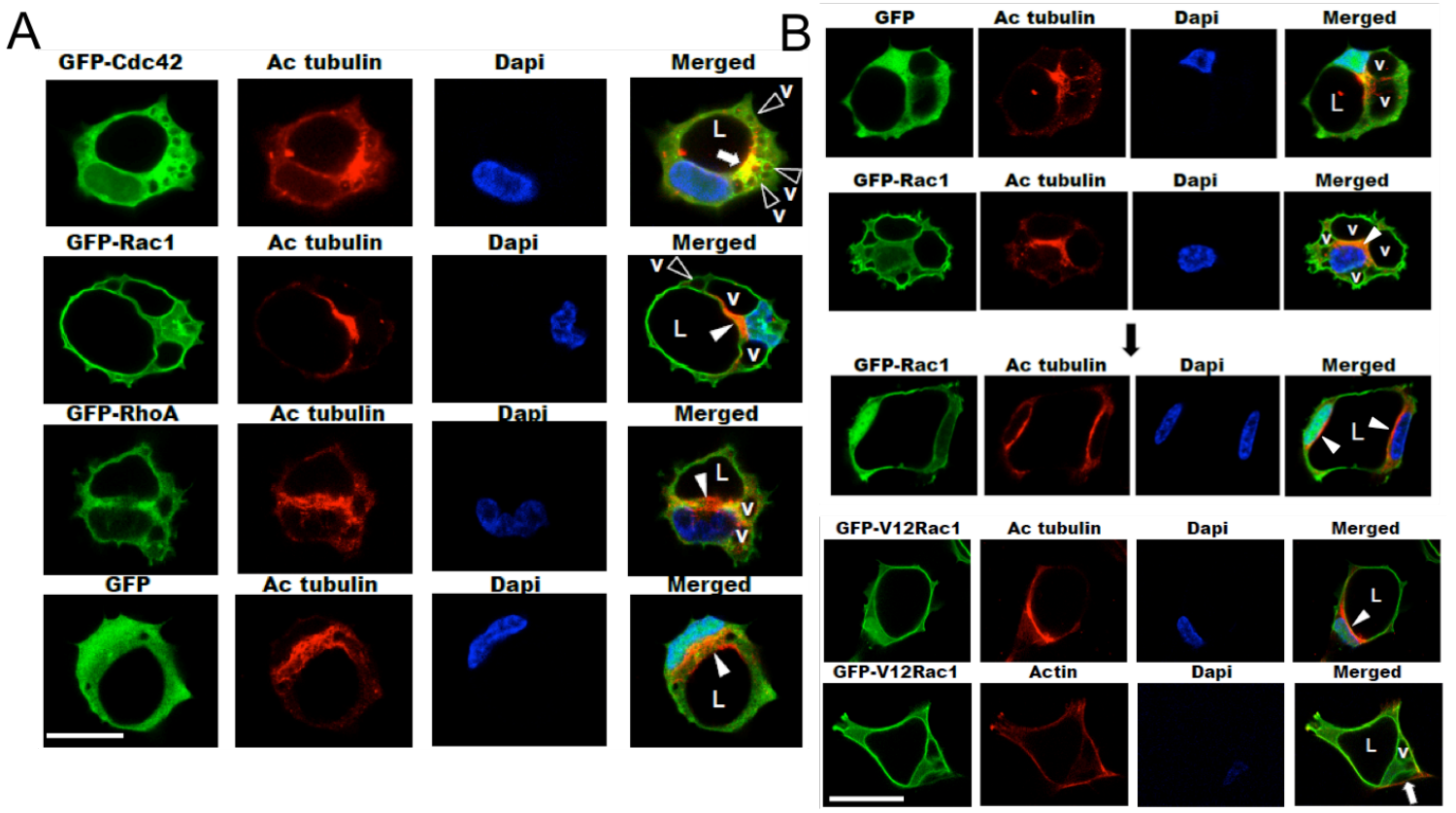

Figure 2.3. Apical polarization of $C d c 42, R a c 1$ and acetylated tubulin during $E C$ tubulogenesis: Membrane trafficking of vacuoles along microtubule tracks toward the apical surface directs EC lumen formation in $3 D$ matrices. (A) ECs were infected with recombinant adenoviruses carrying GFP-Cdc42, GFP-Rac1, GFP-RhoA, or GFP and were allowed to form vacuoles and lumens in $3 \mathrm{D}$ collagen matrices for $12-16 \mathrm{hr}$. to visualize early events in this process. Fixed cultures were then immunostained for acetylated tubulin, analyzed by confocal microscopy, and representative images are shown. White arrowheads indicate subapically polarized acetylated tubulin staining, black arrowheads indicate vacuoles (v). White arrow indicates co-localization of Cdc42 with acetylated tubulin expression in a polarized subapical domain. Vacuoles appear to be in contact with tubulin cytoskeletal tracks that are enriched in acetylated tubulin that coordinate vacuole transport and fusion events at the EC apical membrane surface. L indicates the EC lumen space. Bar equals $25 \mu \mathrm{m}$. (B) ECs were infected with recombinant adenoviruses carrying GFP-Rac1, GFP-V12Rac1, or GFP control and after 12 (upper two panels) or $16 \mathrm{hr}$. (lower three panels), cultures were fixed and stained with either anti-acetylated tubulin antibodies or with phalloidin to label F-actin. White arrowheads indicate apical polarization of acetylated tubulin and $\mathrm{v}$ indicates vacuoles. $\mathrm{L}$ 
indicates lumen space and the white arrow indicates basal polarization of F-actin. Bar equals $25 \mu \mathrm{m}$.

\section{FIGURE 2.4}
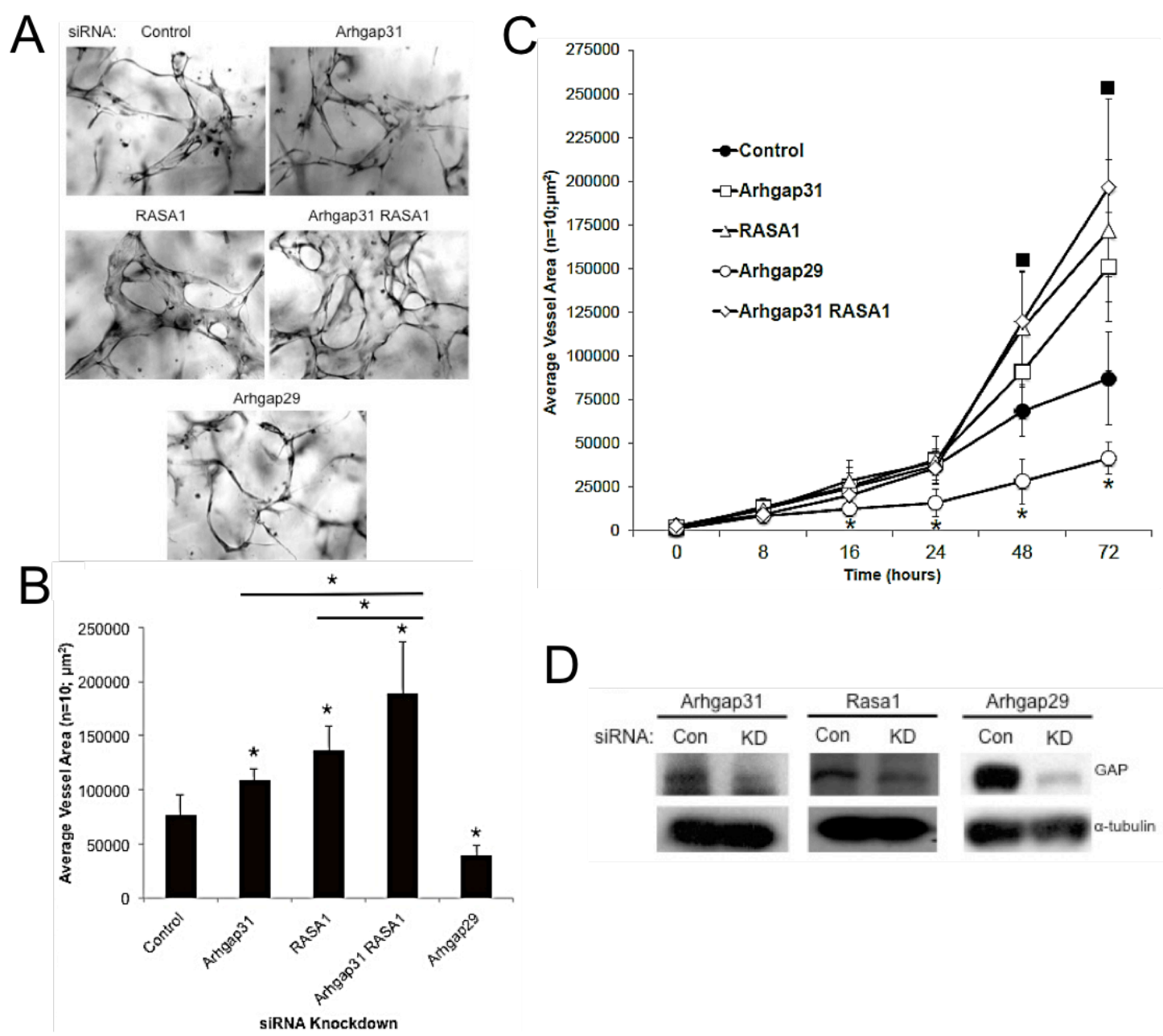

$\mathrm{D}$

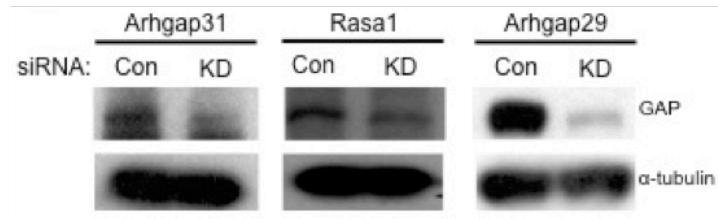

Figure 2.4. EC tubulogenesis is negatively regulated by the GAPs, Arhgap 31 and Rasal, and is positively regulated by Arhgap29. Individual EC cultures were transfected with a control siRNA or siRNAs directed to Arhgap31, Rasa1, Arhgap29, or the combination of Arhgap31 and Rasa1. Treated ECs were then suspended in 3D collagen matrices for 72 $\mathrm{hr}$, were fixed, photographed and lumen formation was quantitated. (A) Representative images of control, Arhgap31, Rasa1, Arhgap31 and Rasa1, and Arhgap29 siRNA transfected EC 3D cultures are shown. Bar equals $100 \mu \mathrm{m}$. (B) Cultures from (A) were quantified for EC tube formation (B). Data are reported as mean vessel area \pm SD per HPF $(n=10 ; p<0.01)$. Asterisk indicates significance compared to control. (C) ECs were treated with the indicated control or GAP siRNAs, lumen assays were performed, fixed, and quantitated at the indicated time points. Squares indicate significance at $p<.01$ above control while asterisks indicate significance below control $(\mathrm{n}=10)$. (D) siRNA 
transfected EC lysates were examined for the degree of protein knockdown using Western Blot analysis.

\section{FIGURE 2.5}

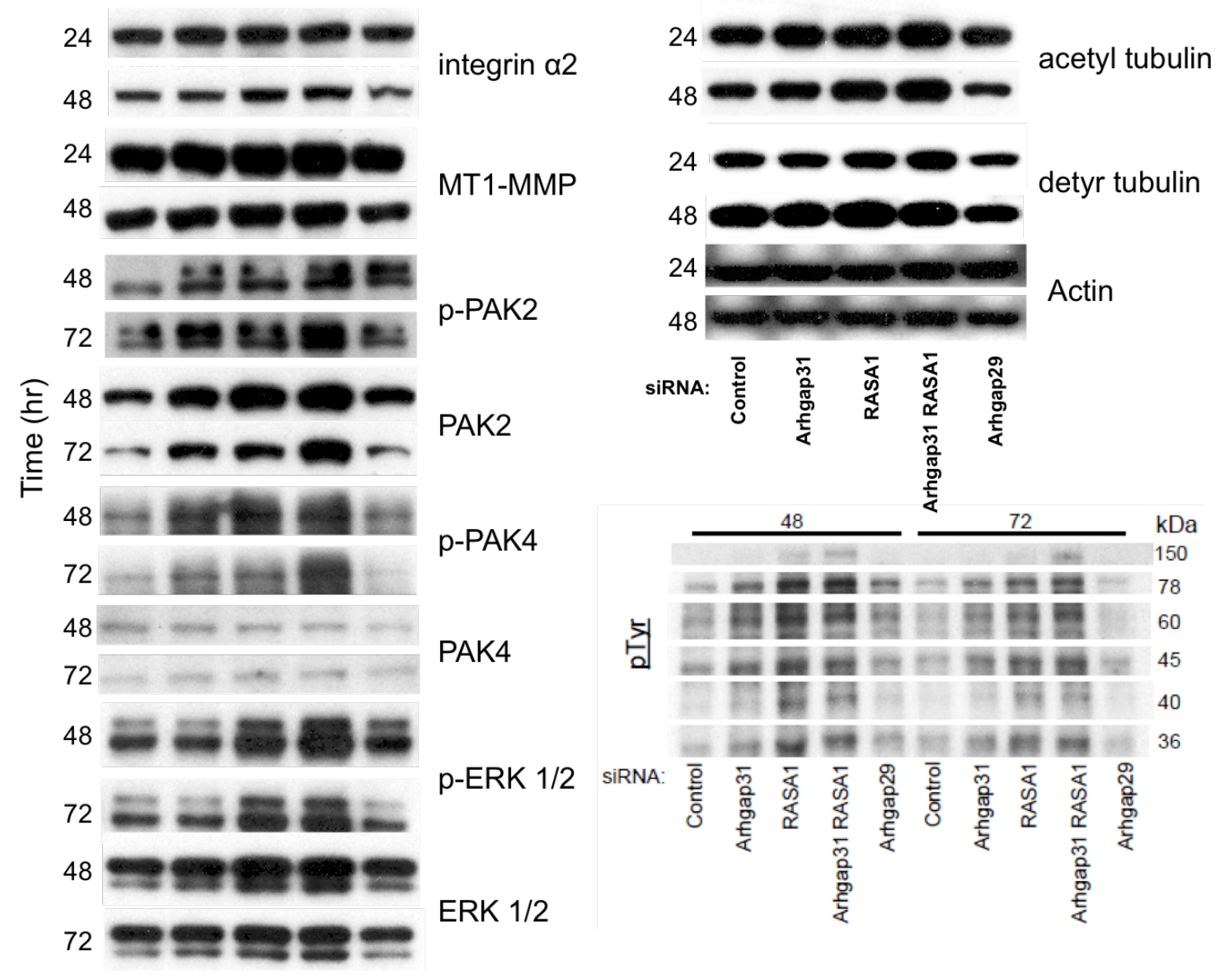

Figure 2.5. Increased $C d c 42, R a c$, and Ras activity via siRNA suppression of Arhgap31, Rasal or both stimulates key signaling pathways and molecules that control EC tubulogenesis. Individual EC cultures were transfected with control siRNA or siRNA targeted against Arhgap31, Rasa1, Arhgap31 and Rasa1, or Arhgap29 and suspended in 3D collagen gel matrices. Lysates were generated at the indicated time points $(24,48$, and $72 \mathrm{hr}$ ) from 3D cultures for Western Blot analysis to assess the expression of $\alpha 2$ integrin, MT1-MMP, p-Pak2, Pak2, p-Pak4, Pak4, p-Erk, Erk, acetylated tubulin, detyrosinated tubulin, tyrosine phosphorylated proteins, and actin over time. Representative blots are shown of 1 of 2 experiments. 


\section{FIGURE 2.6}

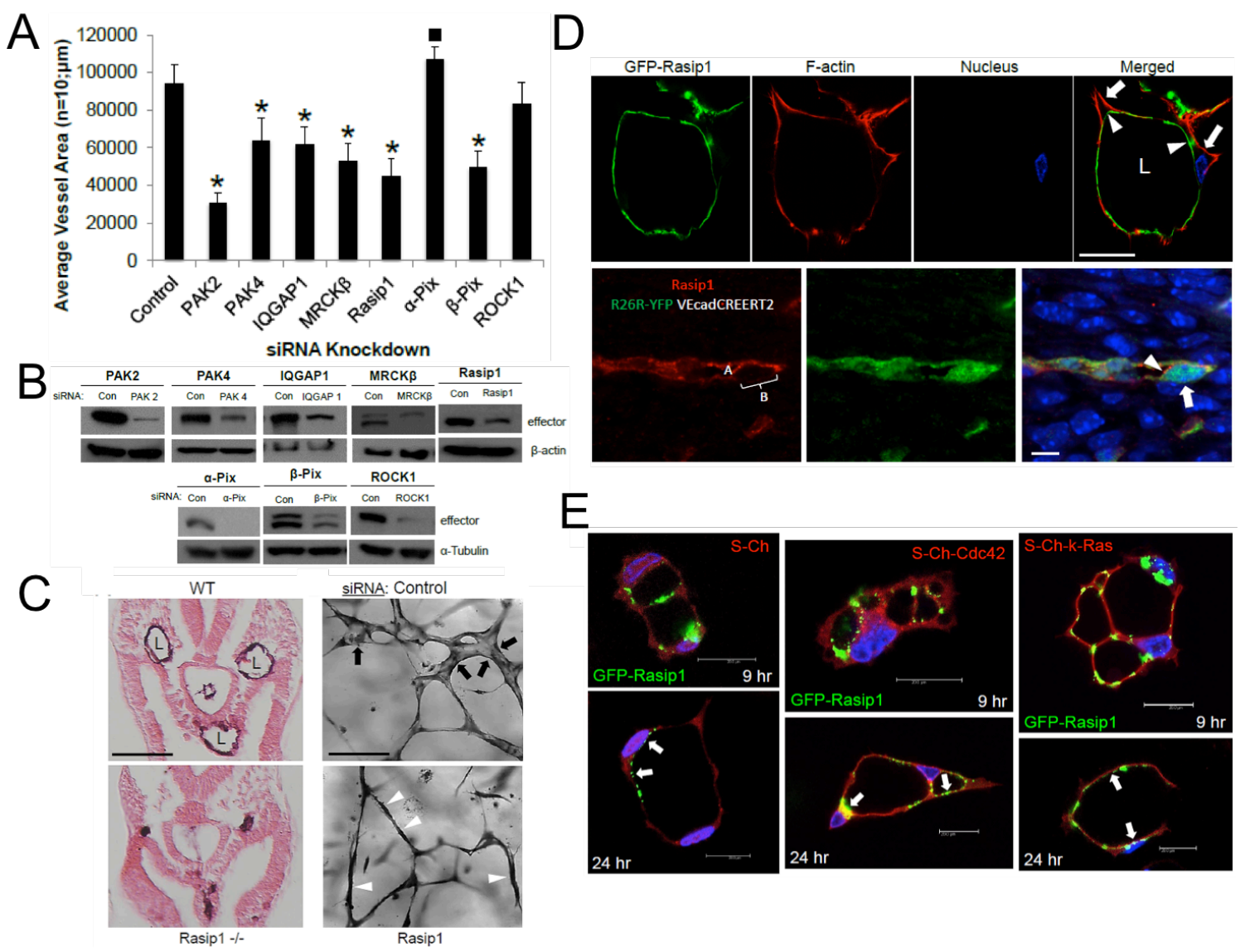

Figure 2.6. IQGAP1, MRCK $\beta$, Rasip1, and beta-Pix are critical downstream effectors of small GTPase signaling that control EC tubulogenesis: Rasipl controls EC tubulogenesis in vivo during mouse development and targets to the EC apical surface in vitro and in vivo. (A) Individual EC cultures were transfected with a control siRNA or siRNAs that are directed to the indicated molecules and were then suspended in $3 \mathrm{D}$ collagen matrices for $72 \mathrm{hr}$. Data are reported as mean vessel area \pm SD per HPF $(n=10 ; p<0.01)$. Asterisk indicates significance below control while square indicates significance above control. (B) Lysates generated from siRNA transfected cultures in (A) were used in Western Blot analysis and probed for the indicated molecules compared to tubulin controls to assess siRNA suppression. (C) Left panels- Wild type vs. Rasip1 knockout mice were cross-sectioned at E8 and were stained with CD31 antibodies. Vascular lumens (L) are observed in the wild type but not the knockout mice. Bar equals $500 \mu \mathrm{m}$. Right panels- ECs were treated with control vs. Rasip1 siRNAs, were seeded in 3D collagen matrices, and after $72 \mathrm{hr}$, were fixed, stained and photographed. Black arrows indicate EC tubes with lumens, while white arrowheads indicate EC cords without lumens mimicking the in vivo observations seen in the left panels. Bar equals $100 \mu \mathrm{m}$. (D) Rasip1 is shown to target to the EC apical surface during tubulogenesis in vitro vs. in 
vivo. Upper panel- ECs were infected with adenoviruses carrying GFP-Rasip1 and PKCe, were cultured in 3D matrices and after $24 \mathrm{hr}$, cultures were fixed, and stained with phalloidin. Arrowheads indicate apical labeled of Rasip1, while the arrows indicate basal labeling of F-actin. Bar equals $25 \mu \mathrm{m}$. Lower panel- Immunofluorescent staining of Rasip1 in developing mouse vessels demonstrates apical targeting of Rasip1.

Arrowheads indicate apical staining of Rasip1 (also labeled A), while the arrow indicates a basal region without staining (also labeled B). Bar equals $5 \mu \mathrm{m}$. (E) ECs were induced to express GFP-Rasip1, mCherry (Ch), Ch-Cdc42, and Ch-k-Ras, allowed to undergo lumen formation, then fixed at the indicated times and imaged by confocal microscopy. Arrows indicate apical and sub-apical targeting of Rasip1 during different stages of EC lumen formation. Bars equal $20 \mu \mathrm{m}$.

\section{FIGURE 2.7}

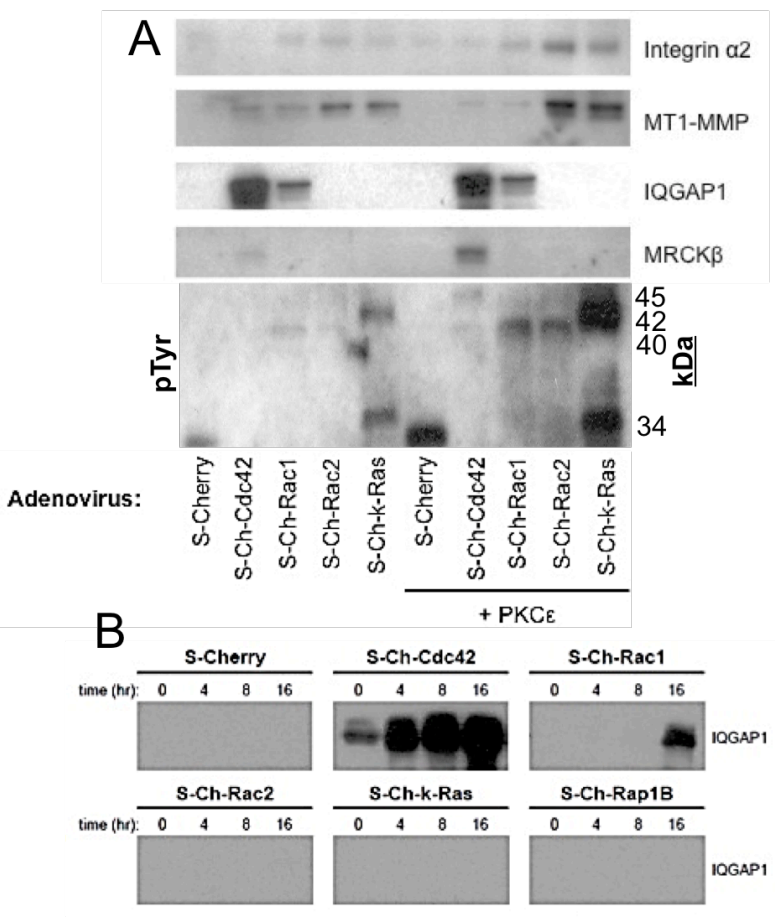

Figure 2.7. Cdc42, Rac1, Rac2, and k-Ras co-associate with a2 integrin, MT1-MMP and key GTPase effectors in lumen signaling complexes during EC tubulogenesis in $3 D$ collagen matrices. (A) ECs were induced to express S-Cherry, S-Ch-Cdc42, S-Ch-Rac1, S-Ch-Rac2, S-Ch-k-Ras, and with or without PKCe. Cultures were then suspended in 3D collagen matrices for 16 hours, detergent lysates from these cultures were then prepared and incubated with S-protein agarose to selectively bind S-epitope tagged proteins and their associated proteins. Eluates were evaluated using Western blot analysis and probed for expression of $\alpha 2$ integrin, MT1-MMP, IQGAP1, MRCK $\beta$, and tyrosine phosphorylated proteins. (B) EC cultures were induced to express S-Cherry, S-Ch-Cdc42, S-Ch-Rac1, S-Ch-Rac2, S-Ch-k-Ras, and S-Ch-Rap1B. Cultures were then suspended in 
3D collagen matrices and detergent lysates were prepared at $0,4,8$, and $16 \mathrm{hr}$. Lysates were incubated with S-protein agarose and eluates were examined for expression of IQGAP1.

\section{FIGURE 2.8}

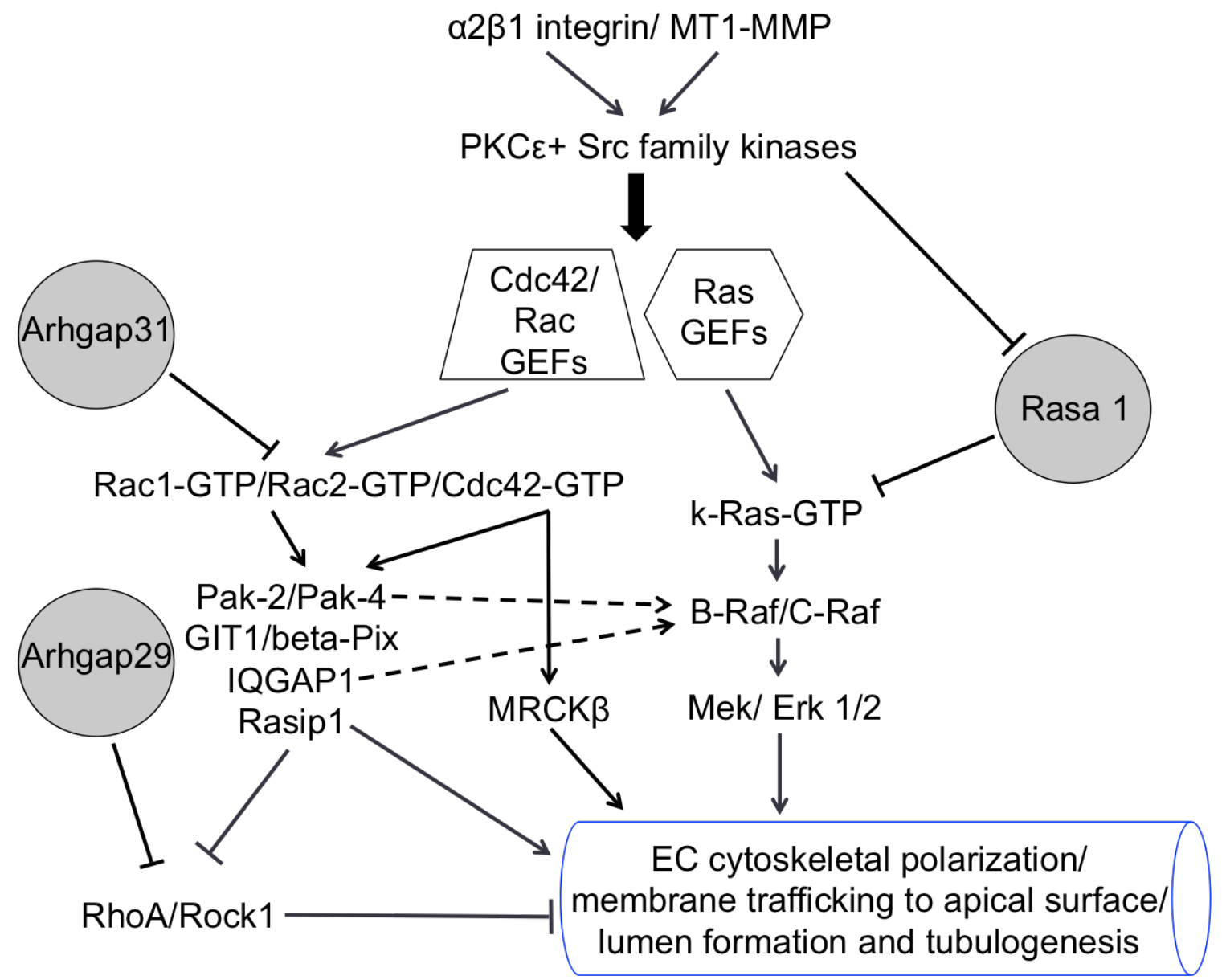

Figure 2.8. EC tubulogenesis in $3 D$ collagen matrices is controlled by activation of a Cdc42-, Rac-, and k-Ras-dependent signaling cascade: A process that is antagonized by Arhgap31, Rasal and RhoA. A schematic diagram is shown illustrating key molecules and signals that underlie how ECs form lumens and tubes in 3D collagen matrices. The indicated molecules and signals allow ECs to form tube networks in 3D matrices and become polarized with respect to the cytoskeleton (acetylated and detyrosinated tubulinapical; F-actin- basal), and the apical membrane which is decorated with key small GTPases that control these processes including Cdc42, Rac1, Rac2, k-Ras and Rap1b. 


\section{SUPPLEMENTAL FIGURES}

\section{SUPPLEMENTAL FIGURE 2.1}

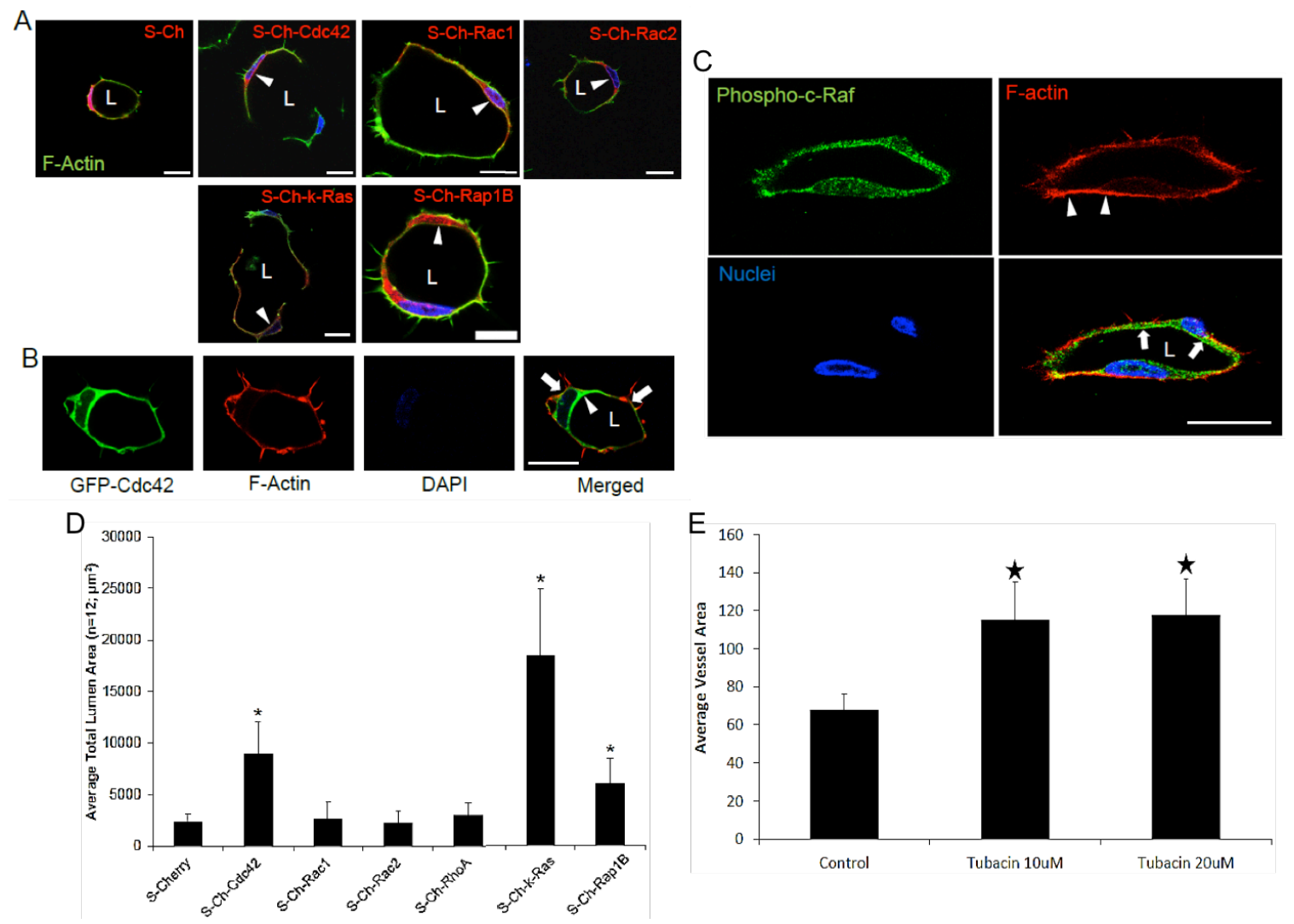

Supplemental Figure 2.1. Apical Polarization of small GTPases and effectors that control EC tubulogenesis. ECs were induced to express Cherry control, the indicated Cherry-GTPases or GFP-Cdc42 fusion proteins and lumen formation assays were performed for 24 hrs. (A,B) Confocal images reveal apical targeting of the indicated GTPases (arrowheads), and arrows indicate basal targeting of F-actin. Bar equals $25 \mu \mathrm{m}$. (C) Apical targeting of activated c-Raf during EC lumen formation (arrows) compared to basal targeting of F-actin (arrowheads). Bar equals $50 \mu \mathrm{m}$. (D) Increased expression of Cdc42, k-Ras and Rap1b leads to accelerated lumen formation. (E) The HDAC6 inhibitor, Tubacin, stimulates EC lumen formation. Asterisks indicates significance at $\mathrm{p}<.01$ compared to control $(\mathrm{n}=12)$. 


\section{SUPPLEMENTAL FIGURE 2.2}

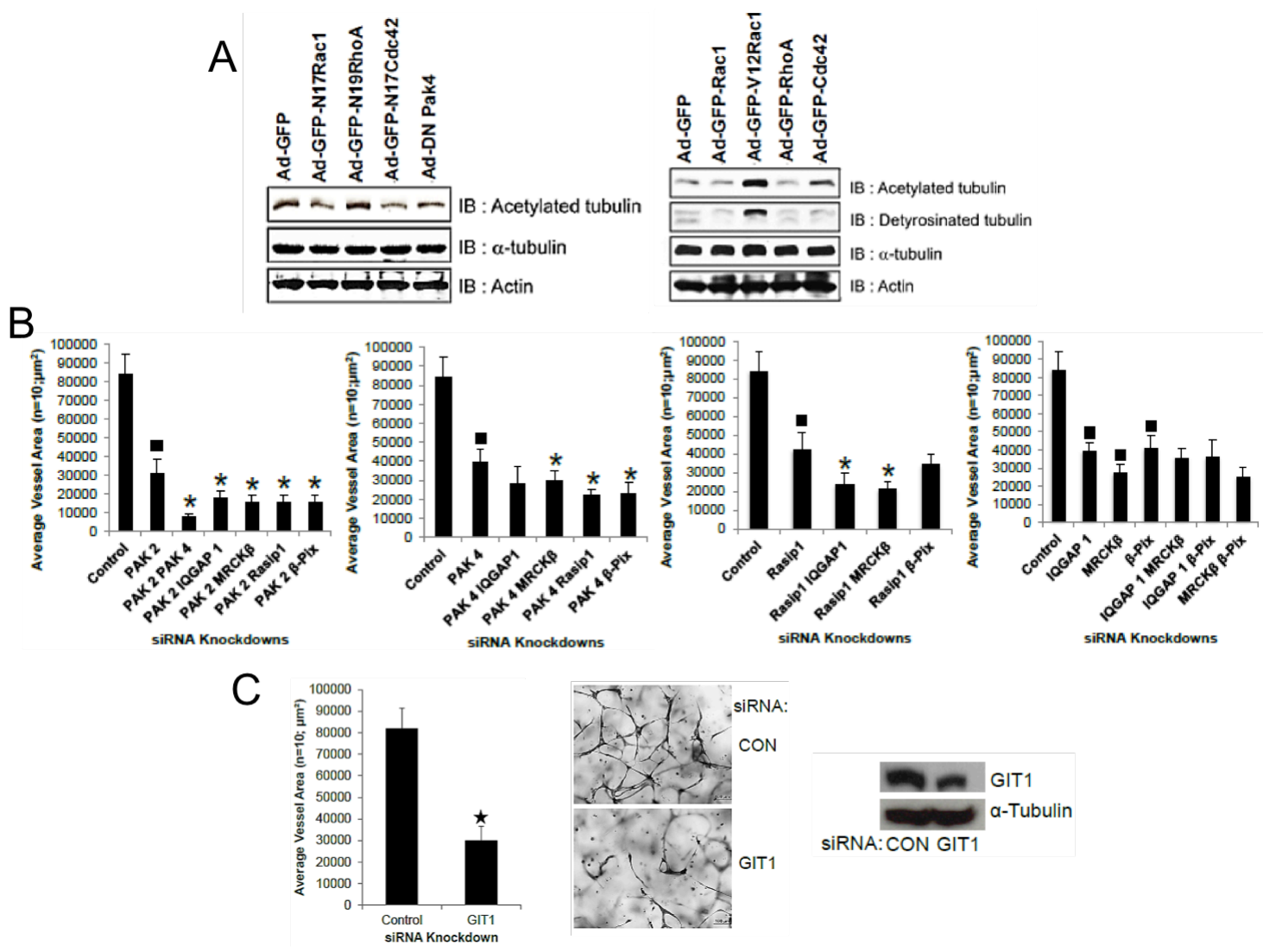

Supplemental Figure 2.2. Cdc42- and Rac-dependent signaling affect tubulin modifications that control EC lumen formation and apical polarization: Key role of small GTPase effectors during EC tubulogenesis. (A) Left panel- ECs were induced to express the indicated dominant negative mutants and lumen cultures were lysed and probed with acetylated tubulin vs. control antibodies. Right panel- ECs were induced to express the indicated wild-type proteins or constitutively active Rac1 mutant and lumen cultures were lysed and probed with acetylated tubulin, detyrosinated tubulin or control antibodies. (B) ECs were treated with the indicated siRNAs, singly or in combinations of two, and EC lumen assays were performed, fixed, photographed and quantitated. Squares indicate significance at $\mathrm{p}<.01$ compared to control, while asterisks indicate significance at $\mathrm{p}<.01$ compared to the indicated single siRNA $(\mathrm{n}=10)$. (C) siRNA suppression of GIT1 reveals a role in EC tubulogenesis. Asterisk indicates significance at $\mathrm{p}<.01$ compared to control. Bar equals $100 \mathrm{~mm}$. 


\section{SUPPLEMENTAL FIGURE 2.3}

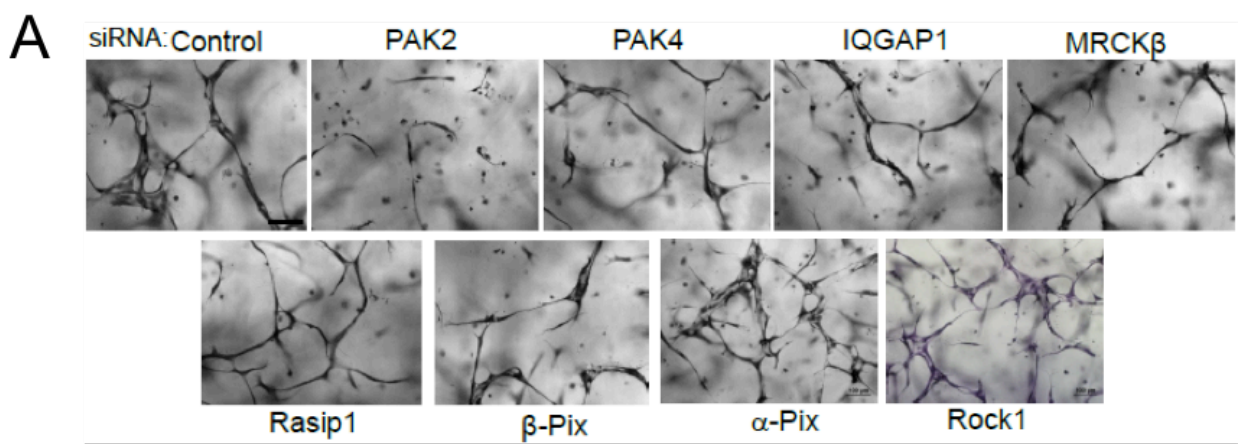

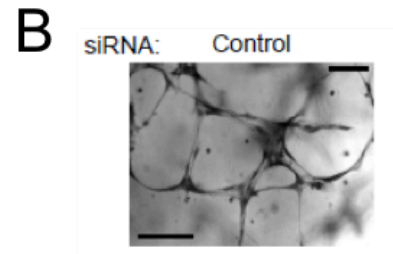

PAK 2 Rasip1

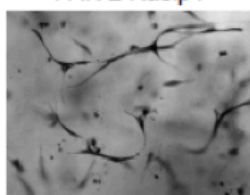

PAK 4 Rasip1

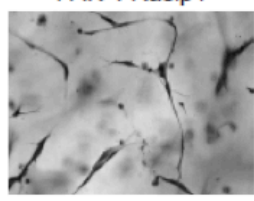

Rasip1 $\beta$-Pix

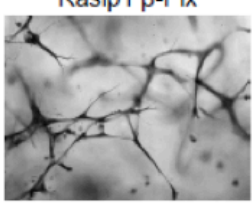

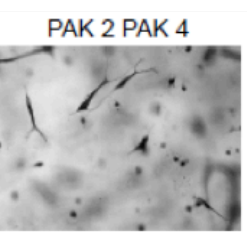

PAK $2 \beta$-Pix

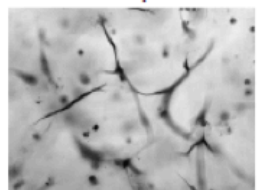

PAK 4 -Pix

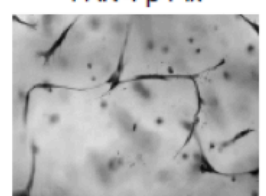

IQGAP 1 MRCK $\beta$

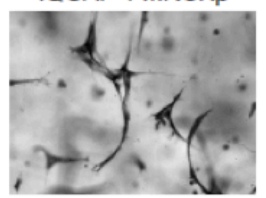

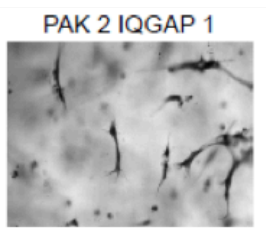

PAK 4 IQGAP 1

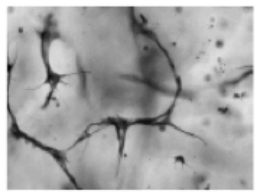

Rasip1 IQGAP 1

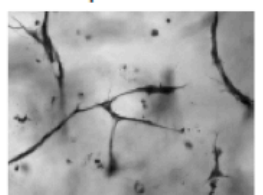

IQGAP $1 \beta$-Pix

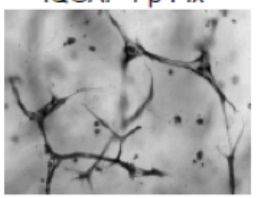

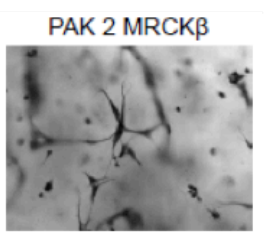

PAK 4 MRCK $\beta$

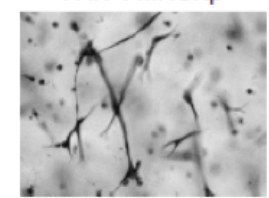

Rasip1 MRCK $\beta$

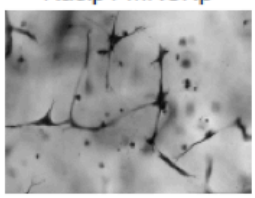

MRCK $\beta \beta$-Pix

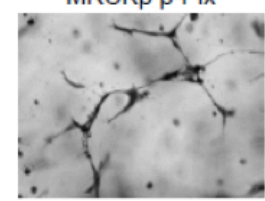

Supplemental Figure 2.3. Small GTPase effectors are fundamental regulators of EC tubulogenesis in $3 D$ matrices. ECs were treated with the indicated siRNAs singly (A) or in combination (B) and tube forming assays were performed and fixed after $72 \mathrm{hr}$.

Representative photographs of the cultures are shown. Bar equals $50 \mu \mathrm{m}$ for $\mathrm{A}$ and 100 $\mu \mathrm{m}$ for B. 


\section{SUPPLEMENTAL VIDEO LEGENDS}

Video S1. ECs were treated with control siRNA and were allowed to form networks of tubes in 3D collagen matrices over $72 \mathrm{hr}$ and in response to the Factors under serum-free media conditions. Considerable movement of the ECs is observed, with intracellular vacuolation and membrane trafficking events controlling the lumen formation process. ECs assemble together through motility towards each other leading to multicellular EC tubes with defined lumens. The video is shown at 7.7 frames/sec.

Video S2. ECs were treated with a Cdc 42 siRNA and were suspended in 3D collagen over $72 \mathrm{hr}$ and in response to the Factors under serum-free media conditions. ECs show motility and move towards each other leading to some cell-cell clusters, but they fail to form sustained lumens and tubes. There are attempts by the cells to form lumen structures but they are unable to sustain them. The video is shown at $7.7 \mathrm{frames} / \mathrm{sec}$.

Video S3. ECs were treated with a RhoA siRNA and were suspended in 3D collagen over $72 \mathrm{hr}$ and in response to the Factors under serum-free media conditions. The treated ECs are readily observed to form lumen and tube structures in a manner similar to control siRNA-treated ECs. The video is shown at 7.7 frames/sec.

Video S4. ECs were treated with an Arhgap31 siRNA and were suspended in 3D collagen over $72 \mathrm{hr}$ and in response to the Factors under serum-free media conditions. The treated ECs are readily observed to form lumen and tube structures in an accelerated manner due to increased Cdc42 and Rac activity compared to control siRNA-treated ECs. The video is shown at 7.7 frames/sec. 
Video S5. ECs were treated with a Rasa1 siRNA and were suspended in 3D collagen over $72 \mathrm{hr}$ and in response to the Factors under serum-free media conditions. The treated ECs are readily observed to form lumen and tube structures in a markedly accelerated manner due to increased Ras activity compared to control siRNA-treated ECs. The video is shown at 7.7 frames/sec.

Video S6. ECs were treated with an Arhgap29 siRNA and were suspended in 3D collagen over $72 \mathrm{hr}$ and in response to the Factors under serum-free media conditions. The treated ECs show the ability to form cords and co-assemble, but attempts at lumen and tube formation are rapidly followed by apparent tube collapse secondary to increased RhoA activity compared to control siRNA-treated ECs. The video is shown at 7.7 frames/sec.

Video S7. ECs were treated with a Rasip1 siRNA and were suspended in 3D collagen over $72 \mathrm{hr}$ and in response to the Factors under serum-free media conditions. The treated ECs show a strong ability to form cords, like we see in in vivo mouse knockout animals, and co-assemble with other ECs. Apparent attempts at lumen formation are rapidly collapsed leaving only networks of cords with no defined lumen space. The video is shown at 7.7 frames/sec.

Video S8. ECs were treated with both Cdc42 and k-Ras siRNAs and were suspended in 3D collagen over $72 \mathrm{hr}$ and in response to the Factors under serum-free media conditions. ECs show reduced motility and some EC-EC interactions, but no lumen and tube formation, so this combination of siRNAs markedly blocks these processes. The video is shown at 7.7 frames/sec. 
Video S9. ECs were treated with both Cdc42 and Rac2 siRNAs and were suspended in 3D collagen over $72 \mathrm{hr}$ and in response to the Factors under serum-free media conditions. ECs show motility, some EC-EC interactions, some interacting cells are seen to come apart, but overall, there is no lumen and tube formation observed. The video is shown at 7.7 frames/sec.

Video S10. ECs were treated with both Cdc42 and Rap1b siRNAs and were suspended in 3D collagen over $72 \mathrm{hr}$ and in response to the Factors under serum-free media conditions. ECs show motility, reduced EC-EC interactions, and interacting ECs appear to disassemble are seen to come apart, but overall, there is no lumen and tube formation observed. The video is shown at $7.7 \mathrm{frames} / \mathrm{sec}$. 


\title{
CHAPTER III
}

\section{RAB AND RAL GTPASES CONTROL ENDOTHELIAL CELL TUBULOGENESIS DURING POLARIZED TRAFFICKING AND FUSION OF CAVEOLIN-1-LABELED PINOCYTIC VACUOLES TO GENERATE THE APICAL MEMBRANE SURFACE}

\author{
Pieter R. Norden ${ }^{1}$, Dae Joong $\mathrm{Kim}^{1}$, and George E. Davis ${ }^{1}$ \\ ${ }^{1}$ Department of Medical Pharmacology and Physiology, University of Missouri School of \\ Medicine, Dalton Cardiovascular Research Center, Columbia, MO 65212
}




\begin{abstract}
Our previous work has shown that EC tubulogenesis requires membrane trafficking steps where pinocytic intracellular vacuoles generated at the basal membrane are trafficked and proceed to undergo fusion with one another in a perinuclear and polarized manner to create the apical luminal membrane. Here, we examine known GTPase regulators of vesicle trafficking events that affect processes such as endocytosis, macropinocytosis, vesicle movement along the cytoskeleton and exocytosis. We identify novel roles for the small GTPases Rab3A, Rab3B, Rab8A, Rab11A, Rab27A, RalA and RalB and Caveolin-1 in co-regulating endothelial cell (EC) lumen and tube formation in 3D collagen matrices. siRNA suppression of individual GTPases such as Rab3A, Rab8A, and RalB markedly inhibit tubulogenesis, while even greater blockade is observed with combinations of siRNAs such as Rab3A and Rab3B, Rab8A and Rab11A, and RalA and RalB. In contrast, knockdown of the endocytosis regulator, Rab5A, fails to inhibit EC tube formation. These GTPases have been strongly implicated in either vesicle trafficking (Rab11A, Rab8A) or membrane exocytosis events (Rab3 and Ral isoforms). Interestingly, Caveolin-1 and RalA strongly label vacuoles and localize to the apical membrane surface and subapical region as they coalesce to form the luminal membrane. In contrast, $\mathrm{Cdc} 42$ and Rab11A strongly localize in a perinuclear, subapical region where intracellular vacuoles accumulate and fuse during lumen formation. Inhibition of Src or Rac activity through use of chemical inhibitors greatly reduces pinocytic intracellular vacuolization and thus markedly impairs EC tubulogenesis whereas inhibition of MT1-MMP activity inhibited fusion of vacuoles/vesicles during EC
\end{abstract}


tubulogenesis implicating an important role for its activity in regulating lumen formation downstream of pinocytic intracellular vacuolization. Thus, EC tubulogenesis is dependent on a series of small GTPases to coordinate polarized membrane trafficking events to generate, deliver, and fuse pinocytic intracellular vacuoles at the EC apical membrane surface during lumen and tube formation. 


\section{INTRODUCTION}

Critical steps during early vasculogenesis and lumen formation include both the establishment of asymmetric cytoskeletal polarization and the trafficking of pinocytic intracellular vacuoles to create an apical membrane surface ${ }^{1-9}$. Importantly, the asymmetric distribution of cytoskeletal components serves a critical function for organization of membrane trafficking to correct locations within the cell ${ }^{10,11}$ and recent work from our laboratory has demonstrated a necessary function for the subapical distribution of tubulin modifications, i.e. acetylated tubulin and detyrosinated tubulin, during EC lumen formation and vasculogenesis ${ }^{7}$ (Chapter II). During vascuologenesis, membrane trafficking events occur along acetylated-tubulin enriched microtubules to organize intracellular vacuoles that coalesce with one another to form a lumen and apical membrane surface and this is controlled in part by the activity of small GTPases and their downstream effectors $^{1,2,7,12-16}$ (Chapter II). An important direction for the advancement of this work is understanding how the necessary steps for lumen formation, i.e. intracellular vacuolization, directed trafficking of vacuoles and vesicles along an asymmetrically polarized cytoskeleton, and coalescence of vacuoles/vesicles to form the lumen, are spatially and temporally controlled by different molecular regulators of membrane trafficking events. Furthermore, a significant challenge in the field has been identifying molecules in ECs that define both the apical membrane surface and the subapical domain and what role they have in regulation of EC lumen formation ${ }^{9}$.

In addition to macropinocytic mechanisms, mammalian cells are capable of transporting membrane compartments through additional endocytic mechanisms, e.g. 
clathrin-mediated, caveolae/caveolin-1 dependent, clathrin independent, phagocytosis and others, as well as through exocytic mechanisms ${ }^{17}$. The Rab family of GTPases comprises the largest family of small GTPases and Rabs are key regulators of membrane trafficking events in eukaryotic cells ${ }^{18-21}$. Recent work in epithelial models of lumen morphogenesis have identified a critical role for Rab11A-Rab8A activity in mediating trafficking of vesicles to the apical membrane initiation site and Rab27/Rab3/Rab8 regulation of vesicle tethering and fusion to form a single lumen during de novo lumen formation ${ }^{22-24}$. In ECs Rab3B, Rab3D, Rab27A and the Ras family GTPase RalA were identified to associate with exocytic secretory granules known as Weibel-Palade bodies that are released from ECs to regulate thrombosis and inflammation ${ }^{25-30}$. Interestingly, it was shown that immunostaining of von Willebrand Factor, a component Weibel-Palade bodies, labeled intracellular vacuole compartments during EC lumen formation ${ }^{31}$, possibly implicating a role for the above exocytic GTPase regulators during EC lumen morphogenesis. Caveolin-1 is also highly expressed in ECs and has been shown to regulate capillary morphogenesis in vitro and in vivo ${ }^{32-34}$. Additionally, caveolae in ECs have been identified to associate with key regulators of EC morphogenesis such as Src, Yes, Raf, MEK, Ras and Rap ${ }^{32,35,36}$. Together, these results implicate a role for Rab and Ral GTPases and Caveolin-1 to control of membrane trafficking events during EC lumen formation and tubulogenesis although the details of this mechanism are unclear.

In this study, we present novel information characterizing the role of regulators of membrane trafficking events during EC lumen formation and tubulogenesis. Our studies have identified novel roles for the GTPases Rab3A, Rab3B, Rab8A, Rab11A, Rab27A, RalA and RalB as well as Caveolin-1 during this process and siRNA suppression of RalB 
combined with Rab3A, Rab3B, Rab27A and RalA markedly impairs the ability of ECs to undergo tubulogenesis. Furthermore, we show that intracellular vacuoles are highly enriched in Caveolin-1 and RalA and we detect targeting of Src to vacuoles as well. Caveolin-1 and RalA then proceed to target to the apical membrane surface while Src and the GTPases Cdc42, Rab11A and Rab27A are localized in the subapical domain. Finally, we show that Src and Rac activity are necessary for the generation of intracellular vacuoles and MT1-MMP activity regulates coalescence of vacuoles downstream of this step. Thus, separate events in EC lumen formation controlling intracellular vacuole generation, polarized trafficking and fusion occur in coordination with key lumen signaling pathways to control how ECs form cell-lined tube networks in 3D extracellular matrices. 


\section{MATERIALS AND METHODS}

\section{Reagents}

Stem cell factor (SCF), stromal cell-derived factor 1 alpha (SDF-1 $\alpha)$ and interleukin-3 (IL-3) were obtained from R\&D systems (Minneapolis, MN). Chemical inhibitors PP3 and EHT 1864 were obtained from TOCRIS Bioscience (Bristol, United Kingdom). Ascorbic acid and 12-O-tetradecanoyl-phorbol-13-acetate (TPA) were obtained from Sigma-Aldrich (St. Louis, MO). Recombinant fibroblast growth factor 2 (FGF-2), antibody against $\beta$-Actin and the chemical inhibitors PP2 and GM6001 were obtained from EMD Millipore (Billerica, MA). Antibodies against Rab3B, Rab3D, Rab11A and Rab27A were obtained from Abcam (Cambridge, MA). Antibodies against Caveolin-1, Rab3A, Rab5 and Rab8 were obtained from Cell Signaling Technology (Danvers, MA). An antibody against RalA was obtained from BD Biosciences (San Jose, CA). GFP-RalA and S-Ch-Cdc42 adenoviruses were generated and utilized as described previously $^{37}$ (Chapter II).

Vasculogenic tube assembly assays

Human umbilical vein endothelial cells were obtained from Lonza (Walkersville, MD) and were cultured (passage 3-6) as described previously ${ }^{38}$.

For use in siRNA-mediated knockdown assays, siRNA transfected ECs were harvested and then suspended at $2 \times 10^{6}$ cells $/ \mathrm{mL}$ in $2.5 \mathrm{mg} / \mathrm{mL}$ collagen type I matrices and assays were performed as described previously ${ }^{39,40}$. In brief, SCF, IL-3, SDF-1 $\alpha$ and FGF-2 were added at $200 \mathrm{ng} / \mathrm{mL}$ directly into collagen type I. Cultures were then fed 
with media containing reduced serum supplement II (RSII), ascorbic acid and FGF-2 at $40 \mathrm{ng} / \mathrm{mL}$. Cultures were allowed to assemble into capillary networks for 72 hours when cultures were fixed in 3\% glutaraldehyde in PBS or collected for additional processing. Following fixation, cultures were then stained in $0.1 \%$ toluidine blue in $30 \%$ methanol for use in imaging and statistical analysis.

For use in confocal microscopy imaging analysis, ECs were first infected with recombinant adenoviruses as described previously ${ }^{12}$. Infected ECs were then harvested and suspended at $2 \times 10^{6}$ cells $/ \mathrm{mL}$ in $3.75 \mathrm{mg} / \mathrm{mL}$ collagen type I matrices and assays were performed as described previously ${ }^{38}$. In brief, cultures were fed with media containing RSII, ascorbic acid, FGF-2 at $40 \mathrm{ng} / \mathrm{mL}$ and TPA at $50 \mathrm{ng} / \mathrm{mL}$. Cultures were allowed to assemble into capillary networks over a period of 0-48 hours when cultures were fixed in 2\% paraformaldehyde in PBS at the indicated time points. Fixed cultures were then stained before use in confocal fluorescent microscopy imaging.

\section{EC siRNA suppression}

siRNA suppression protocols using the siRNA list below were performed as described previously ${ }^{38}$. ECs were allowed to recover for 48 hrs and transfection was repeated. ECs were then allowed to recover overnight before being harvested for use in 3D assays or collected for protein expression analysis using standard western blotting techniques.

siRNAs from Ambion are as follows:

Control (AM4637) Silencer Select Negative Control \#2

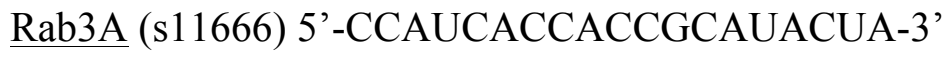


Rab3B (s11669) 5'-GCUUCAUUCUGAUGUAUGA-3'

Rab3D (s18326) 5'-AGGAGAACAUCAAUGUGAA-3'

Rab5A (s11678) 5'-GGAAGAGGAGUAGACCUUA-3'

Rab8A (s8679) 5'-GCAAGAGAAUUAAACUGCA-3'

Rab11A (s16703) 5'-GAGAUUUACCGCAUUGUUU-3'

Rab27A (s11693) 5'-GCCUCUACGGAUCAGUUAA-3'

RalA (s11758) 5'-GGACUACGCUGCAAUUAGA-3'

RalB (s11762) 5'-CAUGAAUCCUUUACAGCAA-3'

Caveolin-1 (s2446) 5'-GCUUCCUGAUUGAGAUUCA-3'

Generation of recombinant adenoviruses

Rab11A and Rab27A were amplified from cDNA obtained from Missouri S\&T cDNA Resource center (Rolla, MO) and mCherry-Rab5A and sfGFP-Caveolin1 fusion constructs were amplified from cDNA obtained from Addgene (Cambridge, MA) and Src was amplified from cDNA obtained from Open Biosystems (Open Biosystems, GE Dharmacon, Lafayette, CO). Standard restriction digest cloning protocol was used to subclone amplified Rab11A and Rab27A into pmCherry-C1 plasmid and Src into pAcGFP-N1 plasmid (Clontech, Mountain View, CA) using EcoRI-HF and BamHI-HF and $X h o I$ and AgeI-HF restriction enzymes respectively (New England Biolabs, Ipswich, MA). Amplified S-epitope (S) tagged S-Ch-Rab5A, S-Ch-Rab11A, S-Ch-Rab27A, SrcGFP-S and sfGFP-Caveolin1 constructs were subcloned into pShuttle-CMV expression plasmid using NotI-HF and XbaI, Sall-HF and NotI-HF and KpnI-HF and NotI-HF restriction enzymes respectively (New England Biolabs). Recombinant adenoviral 
vectors were then generated ${ }^{41}$ and propagated as previously described ${ }^{12}$. The PCR primers used are listed below with the upstream primer first followed by the downstream primer.

Rab11A 5'-AGGAATTCTATGGGCACCCGCGACGACGAGTAC-3'

5'-AGGGATCCTTAGATGTTCTGACAGCACTGCAC-3'

Rab27A 5'-AGGAATTCTATGTCTGATGGAGATTATGATTACC-3'

5'-AGGGATCCTCAACAGCCACATGCCCCTTTCTCC-3'

Src 5'-AGCTCGAGATGgGTAGCAACAAG-3'

5'-AGACCGGTATGAGGTTCTCCCCG-3'

Primers used for subcloning into the pShuttle-CMV plasmid are as follows. The first primer (S-Cherry) is an upstream primer followed by downstream primers for S-ChRab5A, S-Ch-Rab11A and S-Ch-Rab27A. The following sets are pairs of upstream and downstream primers for Src-GFP-S and sfGFP-Caveolin1 respectively.

S-Cherry 5'-AGGCGGCCGCACCATGGCAAAAGAAACCGCTGCTGCGAAATTTG AACGCCAGCACATGGACTCGATGGTGAGCAAGGGCGAGGAG-3'

Rab5A 5'-AGTCTAGATTAGTTACTACAACACTGATTCCTGGTTGGC-3'

Rab11A 5'-AGTCTAGATTAGATGTTCTGACAGCACTGCAC-3'

Rab27A 5'-AGTCTAGATCAACAGCCACATGCCCCTTTCTCC-3'

Src-GFP-S 5'-AGGTCGACATGGGTAGCAACAAGAG-3'

5'- AGGCGGCCGCTTACGAGTCCATGTGCTGGCGTTCAAATTTCGCAGCAG

CGGTTTCTTTACCAGACTTGTACAGCTCATCCATGCCGTG-3’

sfGFP-Caveolin1 5'-AGGGTACCACCATGGTGAGCAAGGGCGAGGAGCTGTTC-3' 5’-AGGCGGCCGCTTATATTTCTTTCTGCAAGTTGATGCGG-3' 
Microscopic imaging and analysis

Fluorescent microscopic imaging of 3D cultures was performed using a confocal microscope (Leica TCS SPE, Leica, Buffalo Grove, IL) using excitation wavelengths of 405 and 488 or 405, 488 and $561 \mathrm{~nm}$ sequentially. High-resolution images were captured using a $63 \mathrm{X}$ water immersion objective (NA 1.2) utilizing Leica application suite advanced fluorescence (LAS-AF) software. Toluidine blue stained 3D cultures were imaged using light microscopy on inverted microscopes (Eclipse TE2000-E; Nikon, Melville, NY with Photometrics CoolSNAPHQ2 camera, Tucson, AZ, and Olympus CKX41 with Olympus DP70 camera, Center Valley, PA). Photographs of cultures were analyzed using Metamorph software (Molecular Devices, Sunnyvale, CA) by tracing vessel area and lumen area.

\section{$\underline{\text { Statistical analysis }}$}

Statistical analysis of selected EC vasculogenic and lumen formation data was performed using Microsoft Excel (Microsoft). Statistical significance was set at minimum with $\mathrm{P}<0.05$. Student's t-tests were used when analyzing two groups within a single experiment (with a minimum $\mathrm{n}=10$ from representative experiments or , consolidated experiments). 


\section{RESULTS}

$\underline{\text { Regulators of vesicle trafficking control EC tubulogenesis in 3D collagen matrices }}$

Recent work in epithelial models of lumen formation demonstrated a required role

for Rab11A-Rab8A regulation of apical trafficking of membrane vesicles ${ }^{22,23}$ and Rab27A/Rab3/Rab8 regulation of vesicle tethering and fusion ${ }^{24}$. Additionally Rab3 and Rab27 were shown to associate with and regulate Weibel-Palade body exocytosis in $\mathrm{ECs}^{26,27,29,30}$ whereas Caveolin-1 has been implicated during angiogenesis events in vitro and in vivo ${ }^{33}$. Therefore, we performed a screen of Rab GTPases and Caveolin-1 during EC tubulogenesis by siRNA suppression and identified several new regulators of membrane trafficking events associated with this process (Fig. 3.1). Our data implicates a key role for Rab3A, Rab3B, Rab8A, Rab11A, Rab27A and Caveolin-1 during this process where Rab3A and Rab8A knockdown have the most marked effects. In contrast, Rab5A knockdown showed no effects whereas Rab3D may be inhibitory as knockdown stimulated EC tubulogenesis. Using combinations of siRNAs, we demonstrated that combined knockdown of Rab8A with Rab11A or Rab27A had a greater blocking effect compared to knockdown of Rab8A alone and that combined knockdown of Rab27A and Caveolin-1 had a greater blocking effect than knockdown of Rab27A or Caveolin-1 alone. Furthermore, these particular combined knockdowns had the greatest blocking effect compared to other siRNA combinations tested (Fig. 3.2 and Fig. 3.3). This data suggests that differential regulation of trafficking events by Rab3A, Rab3B, Rab8A, Rab11A, Rab27A and Caveolin-1 appears to be necessary for ECs to form lumens and 
assemble into capillary networks in 3D matrix environments whereas a role for Rab5A is not supported.

The exocytosis regulators RalA and RalB control EC tubulogenesis

Because we observed strong blocking effects of tubulogenesis with siRNA suppression of Rab3A, Rab3B and Rab27A (Fig. 3.1), we addressed the role of both isoforms of the Ral GTPases, which are known to regulate exocytosis through their association with the members of the exocyst complex, Sec5 and Exo84 $4^{42-47}$ and with Weibel-Palade bodies in $\mathrm{ECs}^{25}$. siRNA suppression of either RalA or RalB blocked EC tubulogenesis with a more prominent effect observed with knockdown of RalB (Fig. 3.4). Using additional combinations of siRNAs, we show that combined knockdown of RalB with Rab3A, Rab3B, Rab27A, and RalA had the greatest blocking effects compared to any of the blocking effects observed with individual siRNA suppression of these GTPases or other combinations (Fig. 3.5 and Fig. 3.6). We also show that combined siRNA suppression of Rab3A and Rab8A has a greater blocking effect than suppression of Rab3A alone (Fig. 3.5 and Fig. 3.6). Together, this data suggests that RalA and RalB are critical regulators of EC lumen morphogenesis. The synergistic effects of RalB knockdown with other GTPases associated with regulation of exocytosis also suggests that these molecules have important, distinct functional roles from one another in control of lumen formation and may operate at different stages. Furthermore, our results from combined siRNA suppression of Rab3A and Rab8A demonstrates a possible role for regulation of differing endocytic and exocytic membrane trafficking steps during EC tubulogenesis. 
Caveolin-1- and RalA-enriched intracellular vacuoles are trafficked from the basal membrane surface to form the apical luminal membrane during EC tubulogenesis

It is necessary during lumen formation for cells to establish an apical membrane surface distinct from the basal membrane surface that interfaces with the extracellular matrix environment. A critical area of focus in our lab is defining how the apical membrane surface is created and stabilized during EC lumen formation and we have made considerable progress in this field of study. We have previously reported that the microtubule plus-end proteins EB1, p150 ${ }^{\text {Glued }}$, and Clasp1 regulate polarization of acetylated tubulin and detyrosinated tubulin subapically during EC lumen formation ${ }^{7}$. We have also demonstrated that acetylated tubulin is highly co-localized with Cdc42 in the subapical domain and that intracellular vacuoles accumulate around this region as they are trafficked along these modified tubulin tracks to the apical membrane surface. Additionally, we also showed that key regulators of lumen morphogenesis, such as Rac1, Rac2, k-Ras, Rap1B, phospho-C-Raf and Rasip1 are localized at the subapical domain or the apical membrane surface during EC tubulogenesis. In contrast, F-actin visualized by staining with fluorescent conjugated phalloidin is polarized in a basal location (Chapter II).

To investigate the subcellular localization of additional key regulators of EC lumen formation and molecules associated with membrane trafficking events, we created a panel of recombinant adenovirus reagents expressing mCherry-fusion or GFP-fusion proteins as shown in Table 3.1. We then used multiple combinations of these reagents to assess whether these molecules co-localize with one another over time during different 
stages of EC lumen morphogenesis. Here, by using GFP-RalA and sfGFP-Caveolin1 constructs we show that Caveolin-1 and RalA strongly localize to intracellular vacuoles generated from the basal membrane surface that are trafficked apically and fuse within a perinuclear region to form the EC apical luminal membrane over time (Fig. 3.7 and Fig. 3.8). Additionally, using a Src-GFP-S construct we show that Src also targets to intracellular vacuoles during this process (Fig. 3.9). As the intracellular vacuoles coalesce to form the lumen during later events, we also show that Caveolin-1 and RalA target to the apical membrane surface while Src is more localized within the subapical domain. In contrast, use of S-Ch-Cdc42, S-Ch-Rab11A, S-Ch-Rab27A and S-Ch-Rab5A constructs showed that Cdc42, Rab11A, Rab27A and Rab5A all show targeting ability to the subapical domain region (Fig. 3.7, Fig. 3.8 and Fig. 3.9). During early EC lumen formation, Rab11A appears to localize to vesicles in a punctate manner that accumulate in a polarized, perinuclear region whereas Rab27A is initially diffuse throughout the cytoplasm and Rab5A is localized to more diffuse, punctate regions. Furthermore, it appears that as intracellular vacuoles are being trafficked apically that they accumulate around these perinuclear regions where Rab11A and Cdc42 are strongly localized.

Pinocytic intracellular vacuolization is required for EC tubulogenesis

Both Rac1 and Src activity have been associated with macropinocytosis ${ }^{48-50}$ and we have previously reported the required activity of Src and Rac1 during EC tubulogenesis in 3D matrices ${ }^{12-14}$. Our lab has also shown that blockade of membrane type I matrix metalloproteinase (MT1-MMP) activity dramatically interferes with EC tubulogenesis by inhibiting the formation of vascular guidance tunnels in 3D matrices ${ }^{51}$. 
Furthermore, MT1-MMP activity is required for Cdc42 activation during EC tubulogenesis ${ }^{15}$. However, whether MT1-MMP activity influences downstream membrane trafficking events during EC tubulogenesis is unclear. To address how Src, Rac, and MMP activity regulate different steps involved in generation, apical trafficking, and fusion of vacuoles during EC lumen formation, we utilized chemical inhibitors in combination with a recombinant adenovirus construct expressing sfGFP-Caveolin 1 and high-resolution confocal microscopy analysis to assess how these separate steps are controlled. We first treated EC cultures with chemical inhibitors targeting Src (PP2 and its negative control PP3), Rac GTPases (EHT 1864), and MMPs (GM 6001) and show that addition of PP2, EHT 1864, or GM 6001 dramatically blocks EC lumen formation after 24 hours of culture (Fig. 3.10A). Confocal microscopy analysis of EC cultures expressing sfGFP-Caveolin 1 construct treated with the above chemical inhibitors show that during early stages of EC lumen morphogenesis, inhibition of Src or Rac activity greatly impairs the ability of ECs to form pinocytic vacuoles, as visualized by Caveolin 1 targeting to vacuoles in Control and PP3 treated cultures, and thus markedly inhibits lumen formation (Fig. 3.10B). In contrast, vacuoles are observed in GM 6001 treated cultures, but they fail to fuse to create the apical luminal membrane (Fig. 3.10B, lower panel). These results suggest that the generation of pinocytic vacuoles is required for EC tubulogenesis and that Rac and Src activity are key regulators of this process. Additionally, MMP activity, likely through MT1-MMP, is required in downstream events controlling either membrane trafficking or vacuole coalescence. 


\section{DISCUSSION}

Intracellular vacuolization is a critical mechanism during EC tubulogenesis and capillary assembly ${ }^{3-6}$. Important to this process is addressing the mechanisms of how ECs are able to generate intracellular vacuoles, traffic them and control fusion of individual vacuoles with one another to create an apical luminal membrane that is matured through continued delivery of intracellular vacuoles and vesicles during the course of lumen formation. In the work presented here, we identify novel roles for Rab3A, Rab3B, Rab8A, Rab11A, Rab27A, RalA and RalB small GTPases and Caveolin-1 in controlling EC lumen formation in coordination with Src, Rac1 and Cdc42 activity as shown in Figure 3.11. We also demonstrate critical roles for key regulators of tubulogenesis in controlling apical-basal polarization of ECs during this process as Caveolin1, RalA and Src target to intracellular vacuoles and the developing apical membrane, whereas Cdc42, Rab11A and Rab27A localize within the subapical domain. Finally, we demonstrate that generation of intracellular vacuoles is necessary for EC lumen formation via Src kinase and Rac activity, whereas MMP activity, likely though MT1-MMP, potentially has an important downstream function in mediating vacuole fusion events in addition to its role in creating vascular guidance tunnels necessary for EC tubulogenesis ${ }^{51}$.

As Rab GTPases are the largest family of small GTPases and regulate a variety of

membrane trafficking events ${ }^{20,21}$, they have become an important target of investigation for studying how membrane material is delivered to a nascent apical domain during de novo lumen formation. Recent work in Madin-Darby Canine Kidney cyst models of epithelial lumen formation have implicated roles for Rab11A-Rab8A and 
Rab27/Rab3/Rab8 pathways in controlling de novo development of lumen cysts ${ }^{22,24}$. However, a role for Rab GTPases in regulation of endothelial lumen formation and tubulogenesis has not been previously reported. Here, we show that siRNA suppression of Rab3A, Rab3B, Rab8A, Rab11A or Rab27A impairs endothelial tubulogenesis in 3D collagen matrices where suppression of Rab3A and Rab8A possess the strongest blocking effects. Additionally, we also show that siRNA suppression of Caveolin-1, RalA and RalB blocks EC tubulogenesis supporting the conclusion that multiple GTPases, as well as Caveolin-1, regulate critical membrane trafficking events necessary for EC tubulogenesis. In contrast, we show evidence suggesting that Rab3 isoforms have important functional differences in this process as siRNA suppression of Rab3D stimulated EC tubulogenesis. However, Rab5A regulation of macropinocytosis or clathrin-mediated endocytosis is not involved, as suppression of Rab5A had no effect on tubulogenesis. In support of the conclusion that these molecules possess different functional roles during different stages of EC tubulogenesis, combined siRNA suppression of Rab8A and Rab11A, Rab8A and Rab27A, and Rab27A and Caveolin1 had stronger blocking effects compared to individual suppression of Rab8A, Rab27A, or Caveolin-1. Additionally, we show that combined siRNA suppression of RalB with Rab3A, Rab3B, Rab27A and RalA markedly blocks tubulogenesis, implicating the critical importance of RalB function during this process. Together, our results show similar functional requirements of Rab3, Rab8A, Rab11A and Rab27A compared to what has previously been reported in MDCK cyst models. In these models, it was shown that Rab11A-Rab8A acts in a signaling pathway to activate Cdc42 activity and association with the Par3/Par6/aPKC complex as well as the exocyst to deliver vesicles apically ${ }^{22}$ 
whereas Synaptotagmin-like protein (Slp)-4a controls vesicle tethering and fusion in conjunction with Rab27/Rab3/Rab8 and the SNARE syntaxin-3 ${ }^{24}$. However, whether these effectors act downstream of Rab3, Rab8A, Rab11A and Rab27A during EC tubulogenesis is unclear. Identification of the molecular mechanisms regulated by these GTPases, Caveolin1, RalA and RalB as well as identification of the mechanisms controlling their activity is of great importance and is the focus of ongoing studies.

Our work presented here also identifies new regulators of EC polarity and trafficking of pinocytic intracellular vacuoles during tubulogenesis. We show that as lumen formation progresses, ECs accumulate intracellular vacuoles generated from the basal membrane surface that are enriched in Caveolin-1 and RalA and we also demonstrate that Src targets to vacuoles. These vacuoles are oriented in a polarized, perinuclear region that proceed to traffic apically and fuse to create the apical luminal membrane where Caveolin-1 and RalA are targeted to. In contrast, we observe that Cdc42, Rab11A, Rab27A and Rab5A target to the subapical domain. We previously demonstrated that during early EC lumen formation, Cdc 42 is highly co-localized with acetylated tubulins as intracellular vacuoles accumulate around this region and are trafficked along modified tubulin tracks (Chapter II). Interestingly, Rab11A appears punctate and highly localized within a similar region suggesting that Rab11A and possibly Rab8A positive vesicles traffic along these modified tubulin tracks and regulate apical trafficking of vacuoles as well, and this will be investigated in future studies. Additionally, Caveolin-1 has been shown to be not only a target of Src phosphorylation ${ }^{52}$ but also a negative regulator of Src kinase activity through cooperation with C-terminal Src kinase (Csk) binding protein ${ }^{53}$. Because both Caveolin-1 and Src target to 
intracellular vacuoles, this suggests that these molecules may interact and potentially regulate the activity of one another. However, further work is required to elucidate their possible mechanism of interaction. We also show by use of chemical inhibitors of Src and Rac that these molecules block lumen formation. This is accomplished by strongly inhibiting pinocytic intracellular vacuolization, which is necessary for EC tubulogenesis, and this reflects results previously reported using chemical inhibitors or siRNA suppression techniques directed to Src and $\mathrm{Rac} 1^{12-14}$. We also present for the first time a possible novel function for MT1-MMP activity in downstream events of EC lumen formation as inhibition of MMP activity did not block pinocytic intracellular vacuolization, but inhibited fusion of vacuoles with one another and expansion of the lumen. The mechanism of this function is unclear and will be pursued in future work. Thus, EC tubulogenesis and lumen formation is a highly coordinated signaling event regulated by a number of GTPases and their downstream effectors with distinct functional differences as supported by the work presented here and what our lab and others have previously reported ${ }^{5,6}$ (Chapter II). However, the molecular mechanisms of these signaling pathways and their interactions with one another are not well understood and it is of great importance in the vascular biology field to continue these investigations so that they may be elucidated. 


\section{REFERENCES}

1. Davis, G.E. and K.J. Bayless, An Integrin and Rho GTPase-Dependent Pinocytic Vacuole Mechanism Controls Capillary Lumen Formation in Collagen and Fibrin Matrices. Microcirculation, 2003. 10(1): p. 27-44.

2. Kamei, M., et al., Endothelial tubes assemble from intracellular vacuoles in vivo. Nature, 2006. 442(7101): p. 453-456.

3. Iruela-Arispe, M.L. and G.E. Davis, Cellular and Molecular Mechanisms of Vascular Lumen Formation. Developmental Cell, 2009. 16(2): p. 222-231.

4. $\quad$ Davis, G.E., et al., Chapter Three - Molecular Basis for Endothelial Lumen Formation and Tubulogenesis During Vasculogenesis and Angiogenic Sprouting, in International Review of Cell and Molecular Biology, W.J. Kwang, Editor. 2011, Academic Press. p. 101-165.

5. $\mathrm{Xu}, \mathrm{K}$. and $\mathrm{O}$. Cleaver, Tubulogenesis during blood vessel formation. Seminars in Cell \& Developmental Biology, 2011. 22(9): p. 993-1004.

6. Sacharidou, A., A.N. Stratman, and G.E. Davis, Molecular Mechanisms Controlling Vascular Lumen Formation in Three-Dimensional Extracellular Matrices. Cells Tissues Organs, 2012. 195(1-2): p. 122-143.

7. Kim, D.J., L.A. Martinez-Lemus, and G.E. Davis, EB1, p150Glued, and Clasp1 control endothelial tubulogenesis through microtubule assembly, acetylation, and apical polarization. Vol. 121. 2013. 3521-3530.

8. Sigurbjornsdottir, S., R. Mathew, and M. Leptin, Molecular mechanisms of de novo lumen formation. Nat Rev Mol Cell Biol, 2014. advance online publication.

9. Davis, G., et al., Endothelial Cell Polarization During Lumen Formation, Tubulogenesis, and Vessel Maturation in 3D Extracellular Matrices, in Cell Polarity 1, K. Ebnet, Editor. 2015, Springer International Publishing. p. 205-220.

10. Macara, I.G., Parsing the Polarity Code. Nat Rev Mol Cell Biol, 2004. 5(3): p. $220-231$

11. Mellman, I. and W.J. Nelson, Coordinated protein sorting, targeting and distribution in polarized cells. Nat Rev Mol Cell Biol, 2008. 9(11): p. 833-45. 
12. Bayless, K. and G. Davis, The Cdc42 and Racl GTPases are required for capillary lumen formation in three-dimensional extracellular matrices. J Cell Sci, 2002. 115(6): p. 1123 - 1136.

13. Koh, W., R.D. Mahan, and G.E. Davis, Cdc42- and Rac1-mediated endothelial lumen formation requires Pak2, Pak4 and Par3, and PKC-dependent signaling. Journal of Cell Science, 2008. 121(7): p. 989-1001.

14. Koh, W., et al., Formation of endothelial lumens requires a coordinated PKC $\epsilon-$, Src-, Pak- and Raf-kinase-dependent signaling cascade downstream of Cdc42 activation. Journal of Cell Science, 2009. 122(11): p. 1812-1822.

15. Sacharidou, A., et al., Endothelial lumen signaling complexes control 3D matrixspecific tubulogenesis through interdependent Cdc42-and MT1-MMP-mediated events. Vol. 115. 2010. 5259-5269.

16. $\mathrm{Xu}, \mathrm{K}$., et al., Blood Vessel Tubulogenesis Requires Rasip1 Regulation of GTPase Signaling. Developmental Cell, 2011. 20(4): p. 526-539.

17. Doherty, G.J. and H.T. McMahon, Mechanisms of endocytosis. Annu Rev Biochem, 2009. 78: p. 857-902.

18. Zerial, M. and H. McBride, Rab proteins as membrane organizers. Nat Rev Mol Cell Biol, 2001. 2(2): p. 107-117.

19. Maxfield, F.R. and T.E. McGraw, Endocytic recycling. Nat Rev Mol Cell Biol, 2004. 5(2): p. 121-32.

20. Stenmark, H., Rab GTPases as coordinators of vesicle traffic. Nat Rev Mol Cell Biol, 2009. 10(8): p. 513-525.

21. Hutagalung, A.H. and P.J. Novick, Role of Rab GTPases in membrane traffic and cell physiology. Physiol Rev, 2011. 91(1): p. 119-49.

22. Bryant, D.M., et al., A molecular network for de novo generation of the apical surface and lumen. Nat Cell Biol, 2010. 12(11): p. 1035-1045.

23. Roland, J.T., et al., Rab GTPase-Myo5B complexes control membrane recycling and epithelial polarization. Proceedings of the National Academy of Sciences, 2011. 108(7): p. 2789-2794.

24. Gálvez-Santisteban, M., et al., Synaptotagmin-like proteins control the formation of a single apical membrane domain in epithelial cells. Nat Cell Biol, 2012. 14(8): p. 838-849. 
25. de Leeuw, H.P., et al., Small GTP-binding protein RalA associates with WeibelPalade bodies in endothelial cells. Thromb Haemost, 1999. 82(3): p. 1177-81.

26. Hannah, M.J., et al., Weibel-Palade bodies recruit Rab27 by a content-driven, maturation-dependent mechanism that is independent of cell type. J Cell Sci, 2003. 116(Pt 19): p. 3939-48.

27. Knop, M., et al., Rab3D and annexin A2 play a role in regulated secretion of $v W F$, but not $t P A$, from endothelial cells. Embo j, 2004. 23(15): p. 2982-92.

28. Rondaij, M.G., et al., Small GTP-binding protein Ral is involved in cAMPmediated release of von Willebrand factor from endothelial cells. Arterioscler Thromb Vasc Biol, 2004. 24(7): p. 1315-20.

29. Lowenstein, C.J., C.N. Morrell, and M. Yamakuchi, Regulation of Weibel-Palade Body Exocytosis. Trends in Cardiovascular Medicine, 2005. 15(8): p. 302-308.

30. Bierings, R., et al., The interplay between the Rab27A effectors Slp4-a and MyRIP controls hormone-evoked Weibel-Palade body exocytosis. Blood, 2012. 120(13): p. 2757-67.

31. Davis, G. and C. Camarillo, An alpha 2 beta 1 integrin-dependent pinocytic mechanism involving intracellular vacuole formation and coalescence regulates capillary lumen and tube formation in three-dimensional collagen matrix. Exp Cell Res, 1996. 224(1): p. 39 - 51.

32. Lisanti, M.P., et al., Characterization of caveolin-rich membrane domains isolated from an endothelial-rich source: implications for human disease. J Cell Biol, 1994. 126(1): p. 111-26.

33. Griffoni, C., et al., Knockdown of caveolin-1 by antisense oligonucleotides impairs angiogenesis in vitro and in vivo. Biochem Biophys Res Commun, 2000. 276(2): p. 756-61.

34. Liu, J., et al., Caveolin-1 expression enhances endothelial capillary tubule formation. J Biol Chem, 2002. 277(12): p. 10661-8.

35. Rizzo, V., et al., Rapid mechanotransduction in situ at the luminal cell surface of vascular endothelium and its caveolae. J Biol Chem, 1998. 273(41): p. 26323-9.

36. Liu, J., et al., Organized endothelial cell surface signal transduction in caveolae distinct from glycosylphosphatidylinositol-anchored protein microdomains. J Biol Chem, 1997. 272(11): p. 7211-22. 
37. Davis, G.E., W. Koh, and A.N. Stratman, Mechanisms controlling human endothelial lumen formation and tube assembly in three-dimensional extracellular matrices. Birth Defects Research Part C: Embryo Today: Reviews, 2007. 81(4): p. 270-285.

38. Koh, W., et al., Chapter 5 In Vitro Three Dimensional Collagen Matrix Models of Endothelial Lumen Formation During Vasculogenesis and Angiogenesis, in Methods in Enzymology, A.C. David, Editor. 2008, Academic Press. p. 83-101.

39. Stratman, A.N., M.J. Davis, and G.E. Davis, VEGF and FGF prime vascular tube morphogenesis and sprouting directed by hematopoietic stem cell cytokines. Vol. 117. 2011. 3709-3719.

40. Bowers, S.L., et al., Investigating human vascular tube morphogenesis and maturation using endothelial cell-pericyte co-cultures and a doxycyclineinducible genetic system in 3D extracellular matrices. Methods Mol Biol, 2015. 1189: p. 171-89.

41. He, T., et al., A simplified system for generating recombinant adenoviruses. PNAS, 1998. 95(5): p. 2509 - 2514.

42. Brymora, A., et al., The brain exocyst complex interacts with RalA in a GTPdependent manner: identification of a novel mammalian Sec3 gene and a second Sec15 gene. J Biol Chem, 2001. 276(32): p. 29792-7.

43. Moskalenko, S., et al., The exocyst is a Ral effector complex. Nat Cell Biol, 2002. 4(1): p. 66-72.

44. Sugihara, K., et al., The exocyst complex binds the small GTPase RalA to mediate filopodia formation. Nat Cell Biol, 2002. 4(1): p. 73-8.

45. Fukai, S., et al., Structural basis of the interaction between RalA and Sec5, a subunit of the sec6/8 complex. Embo j, 2003. 22(13): p. 3267-78.

46. Jin, R., et al., Exo84 and Sec5 are competitive regulatory Sec6/8 effectors to the RalA GTPase. Embo j, 2005. 24(12): p. 2064-74.

47. Shirakawa, R. and H. Horiuchi, Ral GTPases: crucial mediators of exocytosis and tumourigenesis. J Biochem, 2015. 157(5): p. 285-99.

48. Veithen, A., et al., v-Src induces constitutive macropinocytosis in rat fibroblasts. J Cell Sci, 1996. 109 ( Pt 8): p. 2005-12. 
49. Ahram, M., et al., Racl-induced endocytosis is associated with intracellular proteolysis during migration through a three-dimensional matrix. Exp Cell Res, 2000. 260(2): p. 292-303.

50. West, M.A., et al., Rac is required for constitutive macropinocytosis by dendritic cells but does not control its downregulation. Curr Biol, 2000. 10(14): p. 839-48.

51. Stratman, A.N., et al., Endothelial cell lumen and vascular guidance tunnel formation requires MT1-MMP-dependent proteolysis in 3-dimensional collagen matrices. Vol. 114. 2009. 237-247.

52. Li, S., R. Seitz, and M.P. Lisanti, Phosphorylation of Caveolin by Src Tyrosine Kinases: THE $\alpha$-ISOFORM OF CAVEOLIN IS SELECTIVELY PHOSPHORYLATED BY v-SrC IN VIVO. Journal of Biological Chemistry, 1996. 271(7): p. 3863-3868.

53. Place, A.T., et al., Cooperative role of caveolin-1 and C-terminal Src kinase binding protein in C-terminal Src kinase-mediated negative regulation of $c$-Src. Mol Pharmacol, 2011. 80(4): p. 665-72.

54. Schnatwinkel, C., et al., The Rab5 Effector Rabankyrin-5 Regulates and Coordinates Different Endocytic Mechanisms. PLoS Biol, 2004. 2(9): p. e261.

55. Schubert, W., et al., Microvascular hyperpermeability in caveolin-1 (-/-) knockout mice. Treatment with a specific nitric-oxide synthase inhibitor, L-NAME, restores normal microvascular permeability in Cav-1 null mice. J Biol Chem, 2002. 277(42): p. 40091-8.

56. Frank, P.G., et al., Caveolin, Caveolae, and Endothelial Cell Function. Arteriosclerosis, Thrombosis, and Vascular Biology, 2003. 23(7): p. 1161-1168. 


\section{FIGURES AND LEGENDS}

FIGURE 3.1

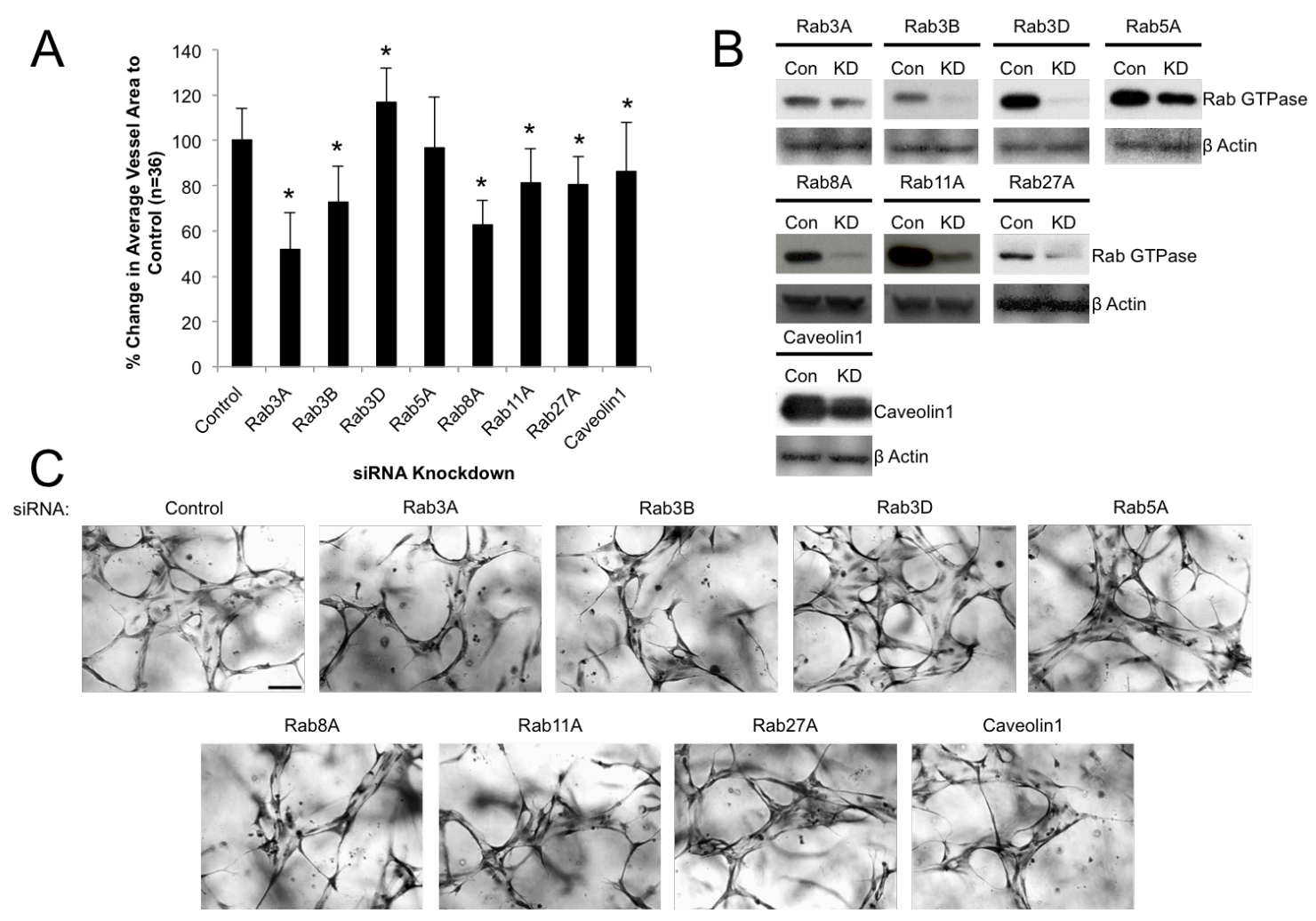

Figure 3.1. Identification of key membrane trafficking regulators that control EC tubulogenesis in $3 D$ matrices. (A) EC cultures were transfected with control siRNA or siRNA directed to Rab3A, Rab3B, Rab3D, Rab5A, Rab8A, Rab11A, Rab27A and Caveolin-1, and then were suspended in 3D collagen matrices for $72 \mathrm{hr}$. before fixation, staining, and photography. Data are normalized to control samples and are reported as average vessel area per high-powered field (HPF) \pm standard deviation (SD) $(n=36, \mathrm{p}<$ .01 ). Asterisk indicates significance compared to control cultures. (B) Lysates generated from transfected cultures in (A) were used in Western blots to assess specific protein knockdown versus control. (C) Representative images from transfected cultures in (A) are shown. Bar equals $100 \mu \mathrm{m}$. 


\section{FIGURE 3.2}
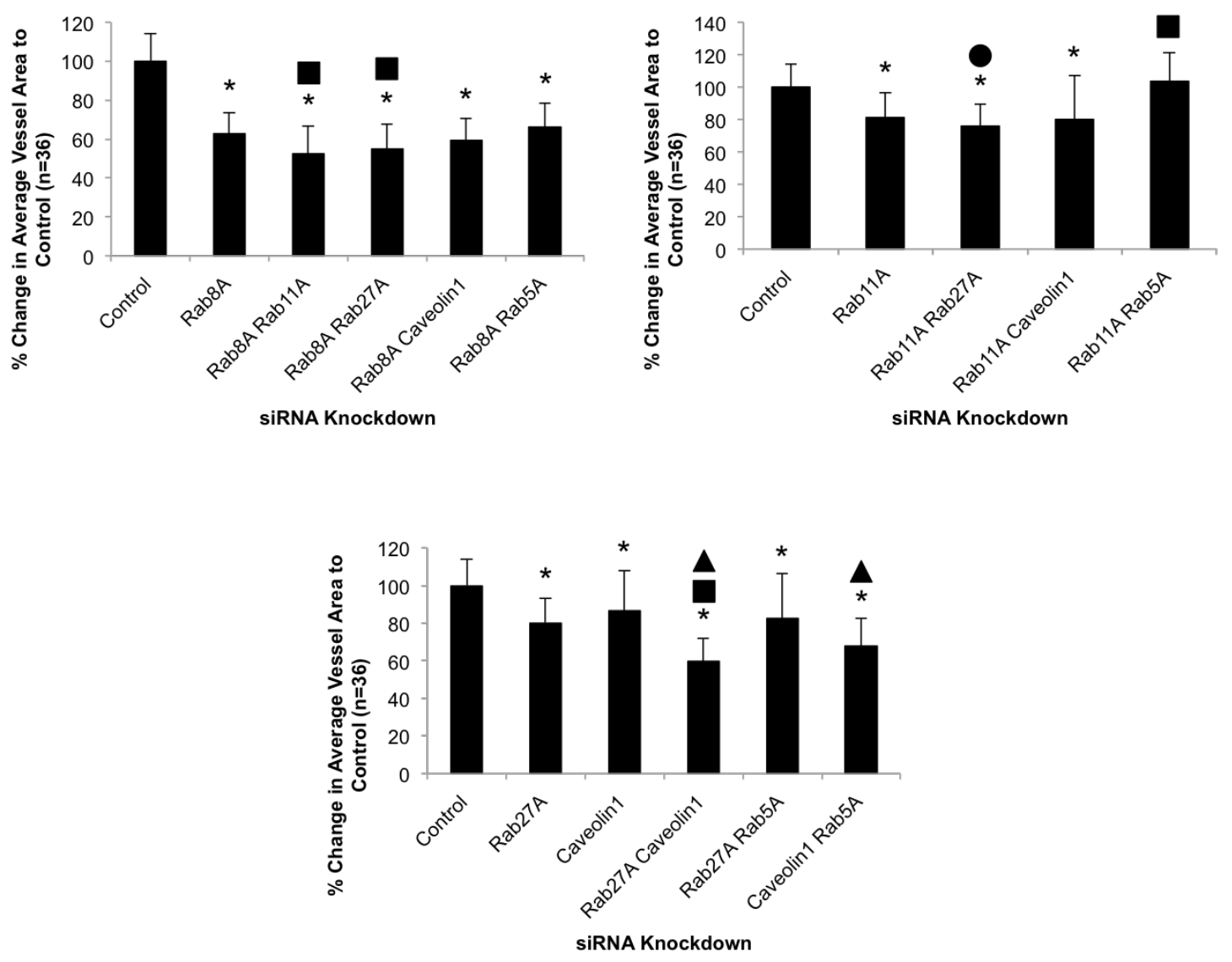

Figure 3.2. Combined siRNA suppression of Rab GTPases and Caveolin-1 delineate functional differences during EC tubulogenesis. Individual EC cultures were transfected with the indicated siRNAs singly or in combinations of two, and suspended in 3D collagen matrices for $72 \mathrm{hr}$. before being fixed, stained, photographed and quantified. Data are normalized to control samples and are presented as average vessel area per HPF $\pm \mathrm{SD}(\mathrm{n}=36)$. Asterisks indicate significance at $\mathrm{p}<.01$ to control whereas squares indicate significance at $\mathrm{p}<.01$ to Rab8A, Rab11A, or Rab27A respectively, circles indicate significance at $\mathrm{p}<.05$ to Rab11A, and triangles indicate significance at $\mathrm{p}<.01$ to Caveolin-1. 


\section{FIGURE 3.3}
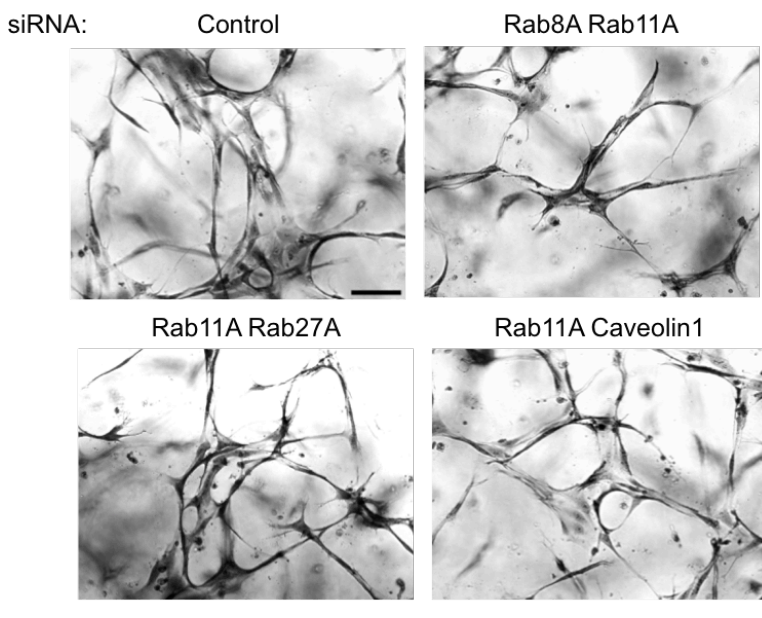

Rab11A Caveolin1
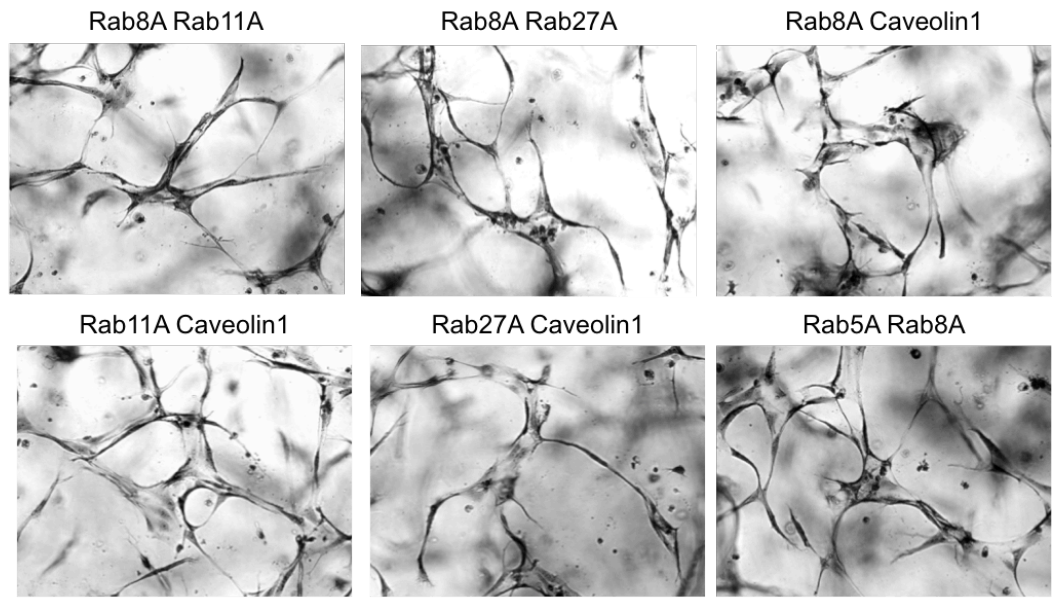

Rab27A Caveolin1

Rab5A Rab8A

Rab5A Rab11A

Rab5A Rab27A
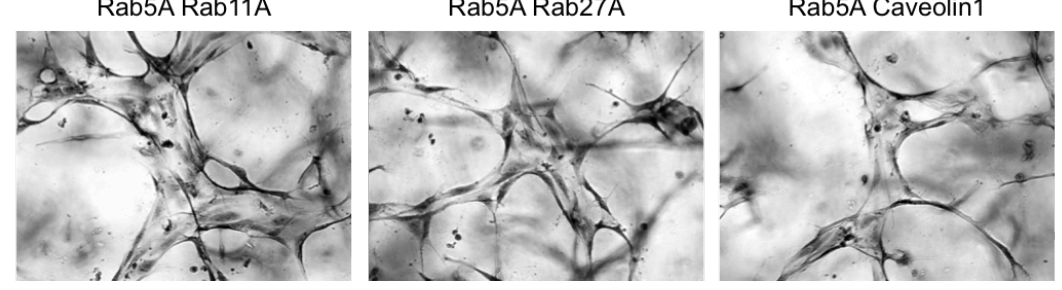

Figure 3.3. EC tubulogenesis requires differential regulation of membrane trafficking events by Rab8A, Rab11A, Rab27A and Caveolin-1. ECs were transfected with control siRNA or the indicated combinations of siRNAs and suspended in 3D collagen matrices for $72 \mathrm{hr}$. before being fixed, stained and photographed. Representative images of cultures are shown. Bar equals $100 \mu \mathrm{m}$. 


\section{FIGURE 3.4}

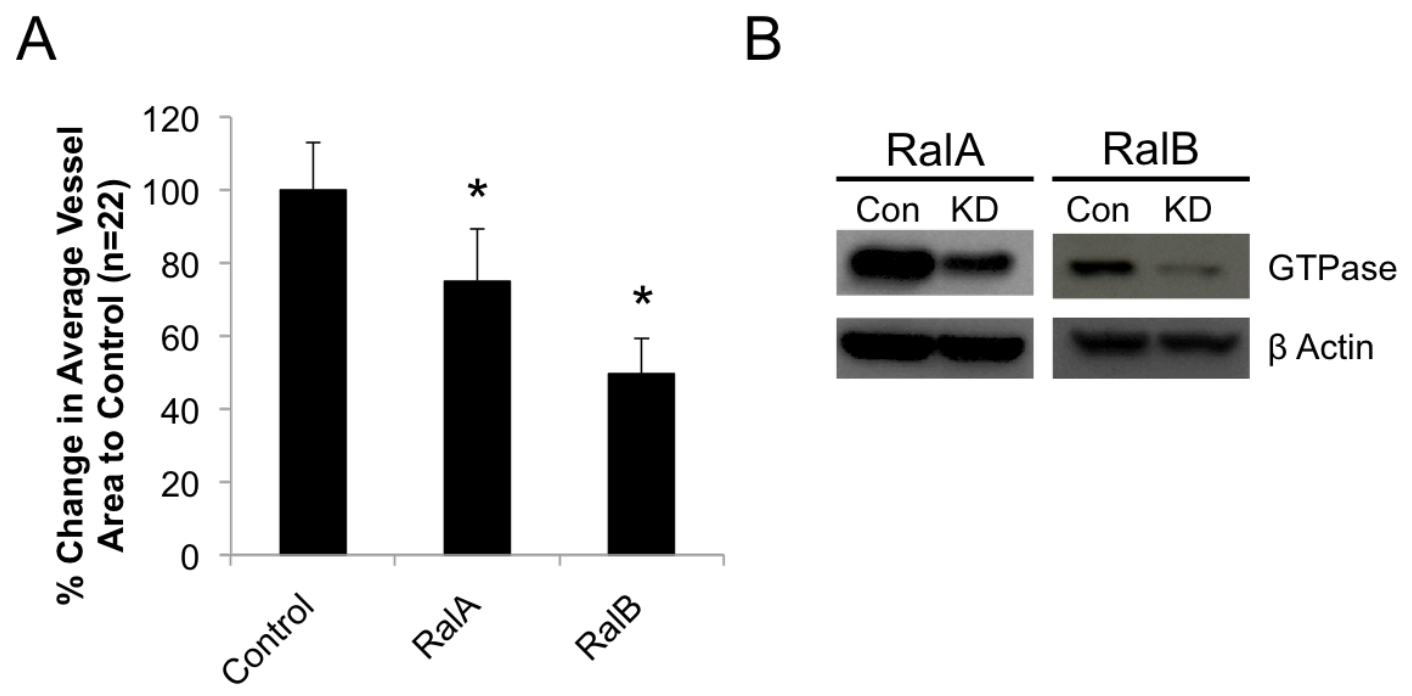

siRNA Knockdown

C

SiRNA:

Control

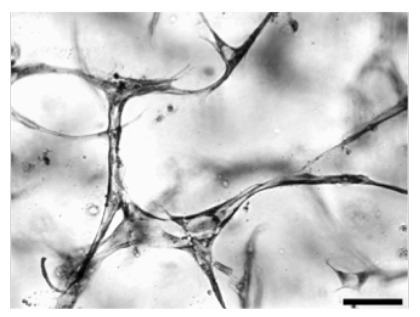

RalA

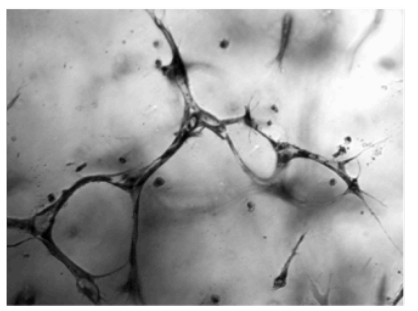

RalB

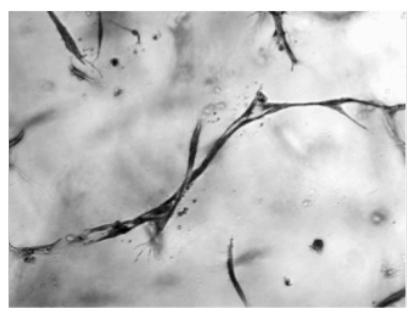

Figure 3.4. RalA and RalB are required for EC tubulogenesis in 3D matrices. (A) EC cultures were transfected with a control siRNA or siRNA directed to RalA or RalB and cultures were suspended in 3D collagen matrices for $72 \mathrm{hr}$. before being fixed, stained, photographed and quantified. Data are normalized to control samples and are reported as average vessel area per HPF $\pm \mathrm{SD}(\mathrm{n}=22, \mathrm{p}<.01)$. Asterik indicates significance to control samples. (B) Lysates generated from transfected cultures in (A) were used in Western blots to assess specific protein knockdown versus control. (C) Representative images from transfected cultures in (A) are shown. Bar equals $100 \mu \mathrm{m}$. 


\section{FIGURE 3.5}
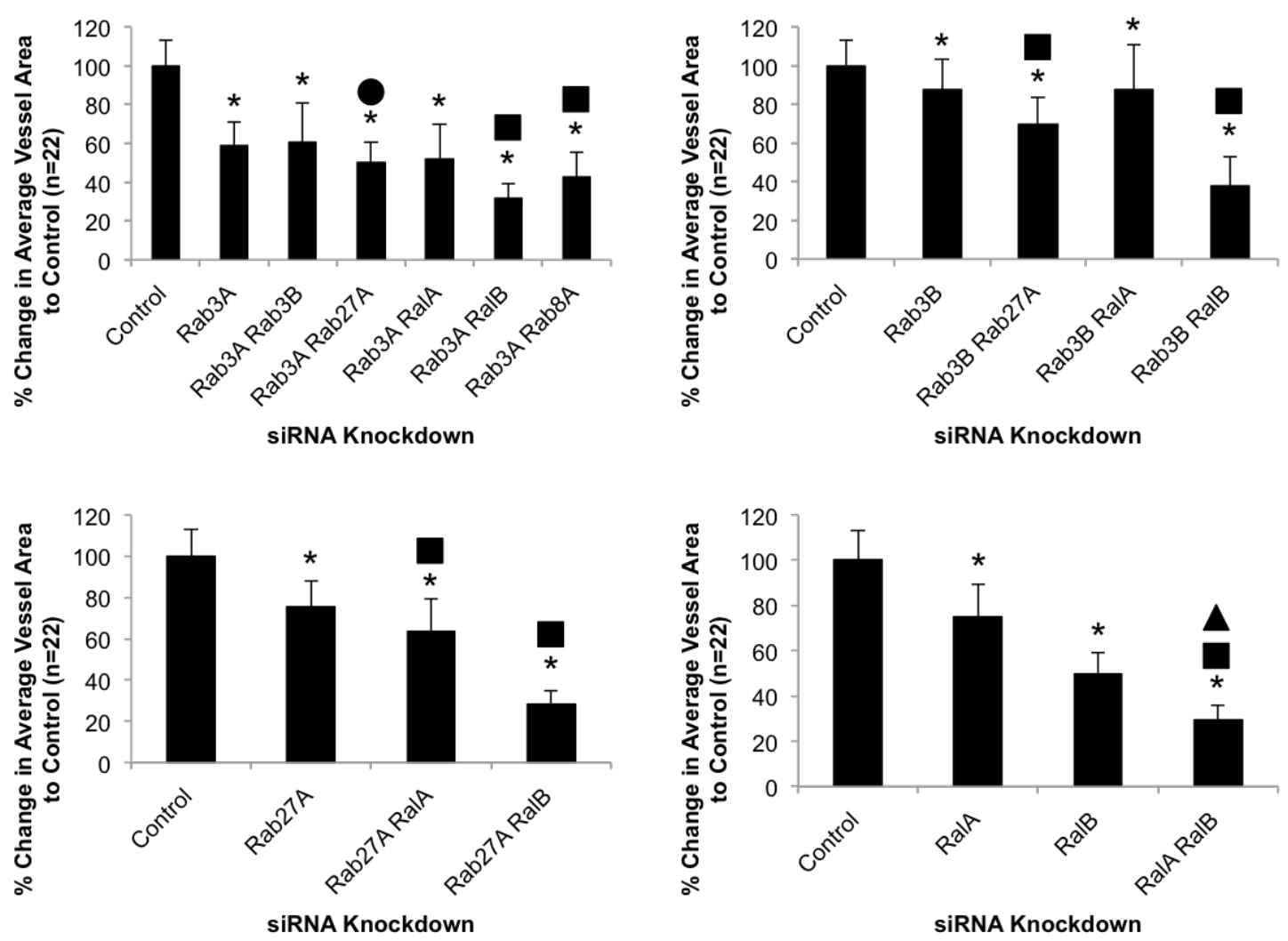

Figure 3.5. Functional differences of small GTPase regulators of exocytosis and endocytosis are critical during EC tubulogenesis in $3 D$ matrices. Individual EC cultures were transfected with the indicated siRNAs singly or in combinations of two, and suspended in 3D collage matrices for $72 \mathrm{hr}$. before being fixed, stained, photographed and quantified. Data are normalized to control samples and are presented as average vessel area per $\mathrm{HPF} \pm \mathrm{SD}(\mathrm{n}=22)$. Asterisks indicate significance at $\mathrm{p}<.01$ to control whereas squares indicate significance at $\mathrm{p}<.01$ to Rab3A, Rab3B, Rab27A, or RalA respectively, circles indicate significance at $\mathrm{p}<.05$ to Rab3A, and triangles indicate significance at $\mathrm{p}<.01$ to RalB. 


\section{FIGURE 3.6}

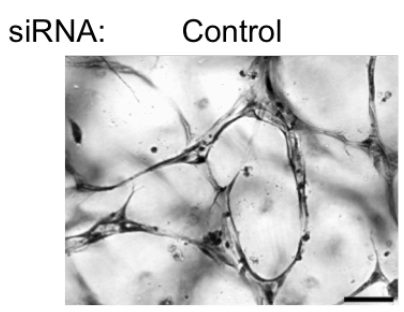

Rab3A RalB

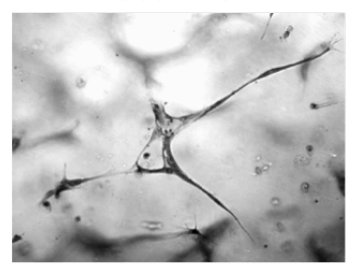

Rab3B RalB

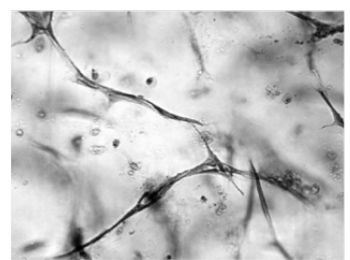

Rab3A Rab3B

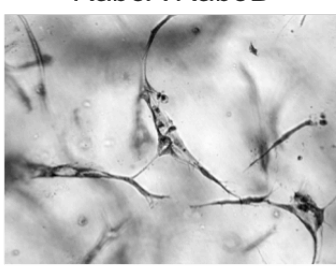

Rab3A Rab8A

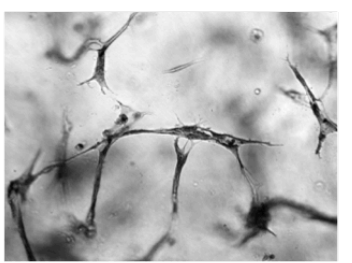

Rab27A RalA

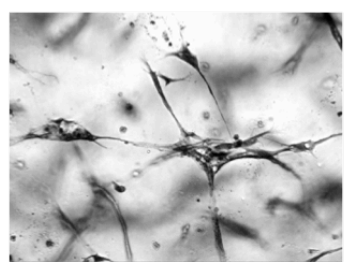

Rab3A Rab27A

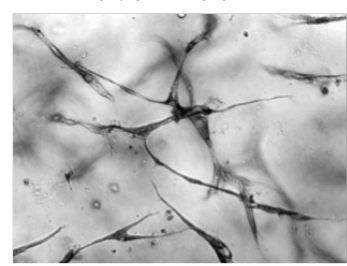

Rab3B Rab27A

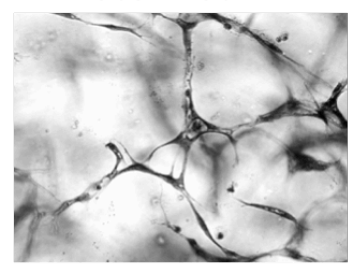

Rab27A RalB

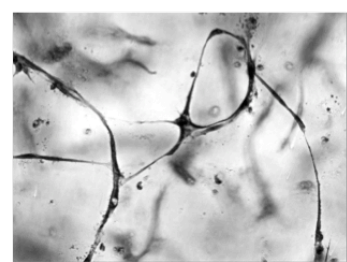

Rab3A RalA

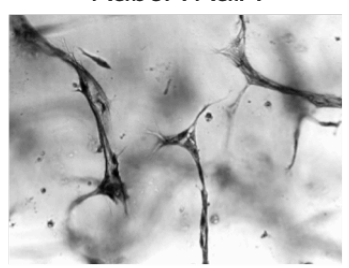

Rab3B RalA

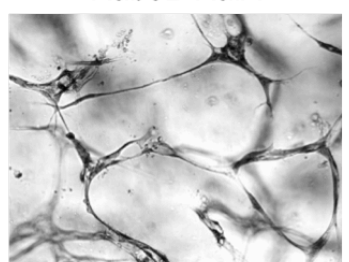

RalA RalB

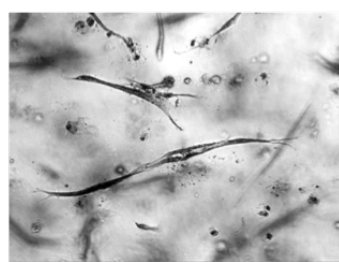

Figure 3.6. RalB is a critical regulator of EC tubulogenesis functionally distinct from Rab3A, Rab3B, Rab27A and RalA. ECs were transfected with control siRNA or the indicated combinations of siRNAs and suspended in 3D collagen matrices for $72 \mathrm{hr}$. before being fixed, stained and photographed. Representative images of cultures are shown. Bar equals $100 \mu \mathrm{m}$. 


\section{TABLE 3.1}

\begin{tabular}{|c|c|c|c|}
\hline $\begin{array}{c}\text { Recombinant } \\
\text { Adenovirus Tools }\end{array}$ & Functional Properties of Proteins & $\begin{array}{l}\text { Cellular Localization During } \\
\text { Endothelial Lumen Formation }\end{array}$ & References \\
\hline S-Ch-Cdc42 & $\begin{array}{l}\text { Required for EC tube formation by association } \\
\text { in signaling complexes with } \alpha 2 \text { integrin, MT1- } \\
\text { MMP, Src, PAK2, PAK4, Par3, Par6b, JamB, } \\
\text { JamC, IQGAP1 and MRCK } \beta\end{array}$ & $\begin{array}{l}\text { Partially localized to intracellular } \\
\text { vacuoles and localizes to the } \\
\text { subapical domain. Strongly co- } \\
\text { localizes with acetylated tubulin. } \\
\text { Co-localizes with Src in the } \\
\text { subapical domain. }\end{array}$ & $\begin{array}{l}\text { Bayless and Davis, } 2002^{12} \\
\text { Kamei et al., } 2006^{2} \\
\text { Koh et al., } 2008^{13} \\
\text { Koh et al., } 2009^{14} \\
\text { Sacharidou et al., } 2010^{15} \\
\text { Norden et al., In revision }\end{array}$ \\
\hline S-Ch-Rab5A & $\begin{array}{l}\text { Implicated in clathrin mediated endocytosis } \\
\text { and early endosomes. May be involved in } \\
\text { macropinocytosis }\end{array}$ & $\begin{array}{l}\text { Punctate distribution in the } \\
\text { cytoplasm in early tube formation } \\
\text { with moderate distribution in a } \\
\text { perinuclear fashion in the subapical } \\
\text { domain later. }\end{array}$ & $\begin{array}{l}\text { Schnatwinkel et al., } 2004^{54} \\
\text { Doherty and McMahon, } 2009^{17} \\
\text { Stenmark, } 2009^{20}\end{array}$ \\
\hline S-Ch-Rab11A & $\begin{array}{l}\text { Required for epithelial cyst and lumen } \\
\text { formation by association with Myosin Vb motor } \\
\text { protein. Activates Rab8A through association } \\
\text { with GEF Rabin8a during epithelial lumen } \\
\text { formation }\end{array}$ & $\begin{array}{l}\text { Punctate distribution in a } \\
\text { perinuclear fashion in early tube } \\
\text { formation and later localizes to the } \\
\text { subapical domain. Exhibits } \\
\text { moderate colocalization with Src in } \\
\text { the subapical domain. }\end{array}$ & $\begin{array}{l}\text { Bryant et al., } 2010^{22} \\
\text { Roland et al., } 2011^{23}\end{array}$ \\
\hline S-Ch-Rab27A & $\begin{array}{l}\text { Involved in exocytic trafficking along with } \\
\text { Rab3A-Rab3D. Involved in Weibel-Palade } \\
\text { body exocytosis. Regulates vesicle tethering } \\
\text { and fusion during epithelial cyst and lumen } \\
\text { formation with Rab3 and Rab8 }\end{array}$ & $\begin{array}{l}\text { Distributed within the cytoplasm } \\
\text { during early morphogenesis and } \\
\text { localizes to the subapical domain } \\
\text { later. Exhibits moderate } \\
\text { colocalization with Src in the } \\
\text { subapical domain. }\end{array}$ & $\begin{array}{l}\text { Hannah et al., } 2003^{26} \\
\text { Stenmark, } 2009^{20} \\
\text { Bierings et al., } 2012^{30} \\
\text { Gálvez-Santisteban et al., } 2012^{24}\end{array}$ \\
\hline GFP-RalA & $\begin{array}{l}\text { Involved in exocytosis through regulation of } \\
\text { the exocyst complex and is association with } \\
\text { Weibel-Palade bodies. Targets to intracellular } \\
\text { vacuoles during EC tube formation }\end{array}$ & $\begin{array}{l}\text { Highly enriched in intracellular } \\
\text { vacuoles and is localized at the } \\
\text { apical membrane surface. }\end{array}$ & $\begin{array}{l}\text { Lowenstein, Morrel, and } \\
\text { Yamakuchi, } 2005^{29} \\
\text { Davis et al., } 2007^{37} \\
\text { Moskalenko et al., } 2012^{43}\end{array}$ \\
\hline Src-GFP-S & $\begin{array}{l}\text { Tyrosine kinase involved in macropinocytosis } \\
\text { and is necessary for EC tube formation and } \\
\text { activation of the Raf/Mek/Erk1/2 kinase } \\
\text { cascade. }\end{array}$ & $\begin{array}{l}\text { Localizes to intracellular vacuoles } \\
\text { and the subapical domain. Exhibits } \\
\text { colocalization with Cdc42, Rab11A } \\
\text { and Rab27A }\end{array}$ & $\begin{array}{l}\text { Doherty and McMahon, } 2009^{17} \\
\text { Koh et al., } 2009^{14}\end{array}$ \\
\hline sfGFP-Caveolin1 & $\begin{array}{l}\text { Component of caveolae and has important } \\
\text { role in transcytosis. Caveolin1 deficient mice } \\
\text { exhibit defects in tight junction formation and } \\
\text { capillary EC adhesion to basement } \\
\text { membrane. Associates with SFKs, Raf, MEK, } \\
\text { Ras, and Rap1 among other proteins in ECs. }\end{array}$ & $\begin{array}{l}\text { Highly enriched in intracellular } \\
\text { vacuoles and strongly localizes to } \\
\text { the apical membrane surface. }\end{array}$ & $\begin{array}{l}\text { Schubert et al., } 2002^{55} \\
\text { Frank, Woodman, and Lisanti, } \\
2003^{56}\end{array}$ \\
\hline
\end{tabular}

Table 3.1. Generation of recombinant adenovirus constructs to assess subcellular localization and polarization of key regulators of EC tubulogenesis and membrane trafficking during EC lumen formation. 


\section{FIGURE 3.7}
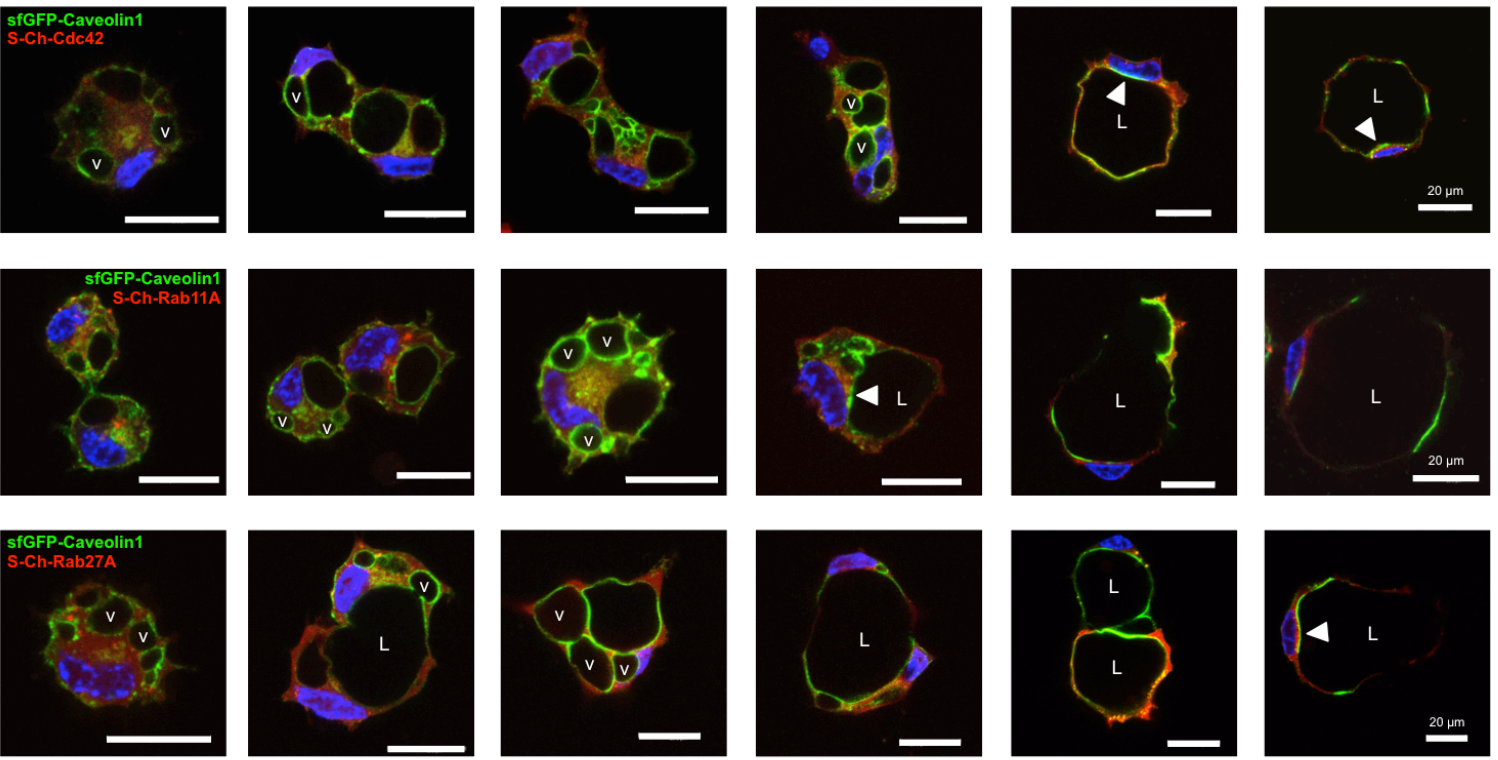

$12 \mathrm{hr}$

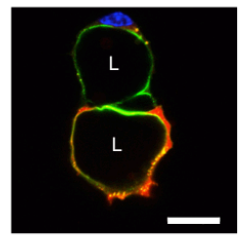

$24 \mathrm{hr}$

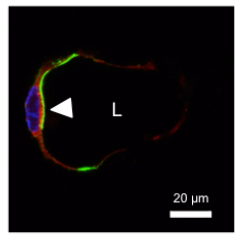

$48 \mathrm{hr}$

Figure 3.7. Caveolin-1 targets to pinocytic intracellular vacuoles and the apical membrane surface during EC tubulogenesis in $3 D$ matrices. ECs were infected with recombinant adenovirus carrying sfGFP-Caveolin1 in combination with recombinant adenoviruses expressing S-Ch-Cdc42 (top row), S-Ch-Rab11 A (middle row), or S-ChRab27A (bottom row) and were allowed to form vacuoles and lumens in 3D collagen matrices for 3-48 hr. to visualize this process. Fixed cultures were then analyzed by confocal microscopy and representative images are shown. White arrowheads indicate targeting of Caveolin-1 to the apical membrane surface and subapical distribution of Cdc42, Rab11A or Rab27A. v indicates representative examples of vacuoles containing Caveolin-1. L indicates the EC lumen space. Bar equals $20 \mu \mathrm{m}$. 
FIGURE 3.8
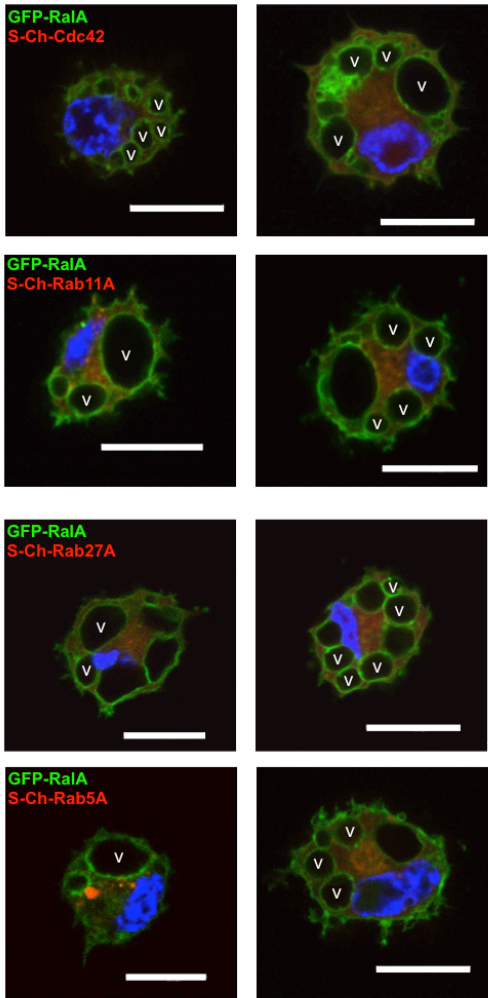

$3 \mathrm{hr}$
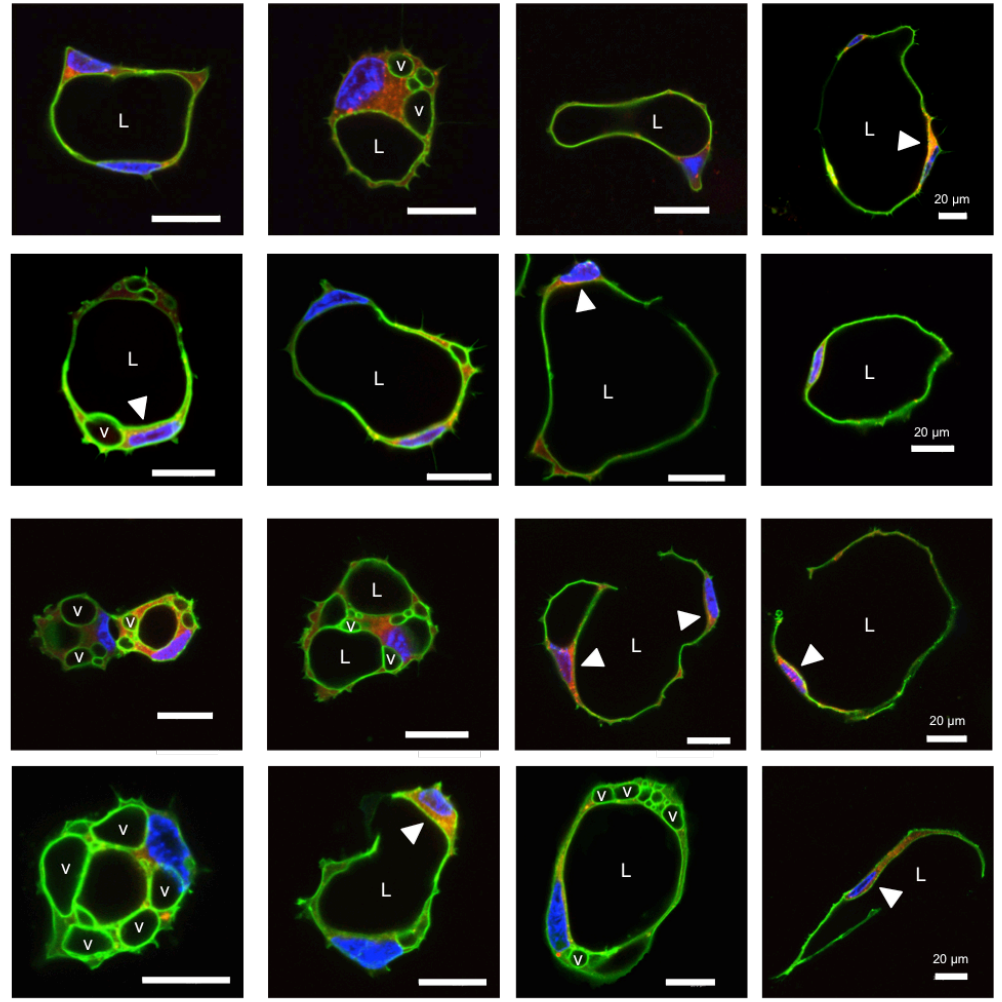

$9 \mathrm{hr}$

$12 \mathrm{hr}$

$24 \mathrm{hr}$

$48 \mathrm{hr}$

Figure 3.8. RalA targets to pinocytic intracellular vacuoles and the apical membrane surface during EC tubulogenesis in $3 D$ matrices. ECs were infected with recombinant adenovirus carrying GFP-RalA in combination with recombinant adenoviruses expressing S-Ch-Cdc42 (first row), S-Ch-Rab11A (second row), S-Ch-Rab27A (third row) or S-Ch-Rab5A (fourth row) and were allowed to form vacuoles and lumens in 3D collagen matrices for 3-48 hr. to visualize this process. Fixed cultures were then analyzed by confocal microscopy and representative images are shown. White arrowheads indicate targeting of Ral-A to the apical membrane surface and subapical distribution of Cdc42, Rab11A, Rab27A or Rab5A. $v$ indicates representative examples of vacuoles containing RalA. L indicates the EC lumen space. Bar equals $20 \mu \mathrm{m}$. 


\section{FIGURE 3.9}
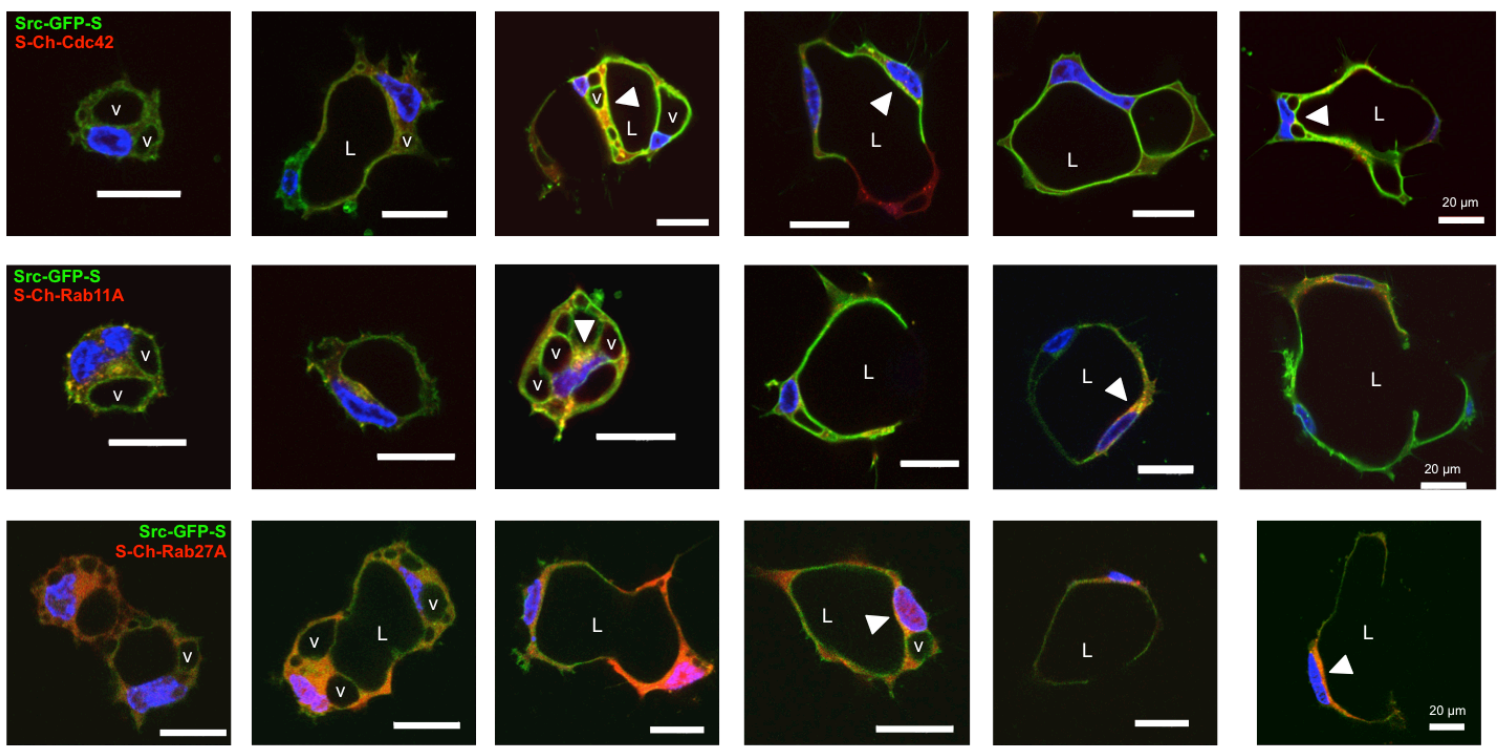

$3 \mathrm{hr}$

$6 \mathrm{hr}$

$9 \mathrm{hr}$

$12 \mathrm{hr}$

$24 \mathrm{hr}$

$24 \mathrm{hr}$

Figure 3.9. Src targets to pinocytic intracellular vacuoles and the subapical domain during EC tubulogenesis in $3 D$ matrices. ECs were infected with recombinant adenovirus carrying Src-GFP-S in combination with recombinant adenoviruses expressing S-ChCdc42 (top row), S-Ch-Rab11A (middle row), or S-Ch-Rab27A (bottom row) and were allowed to form vacuoles and lumens in 3D collagen matrices for 3-24 hr. to visualize this process. Fixed cultures were then analyzed by confocal microscopy and representative images are shown. White arrowheads indicate subapical distribution of Src and Cdc42, Rab11A or Rab27A. v indicates representative examples of vacuoles containing Src. L indicates the EC lumen space. Bar equals $20 \mu \mathrm{m}$. 


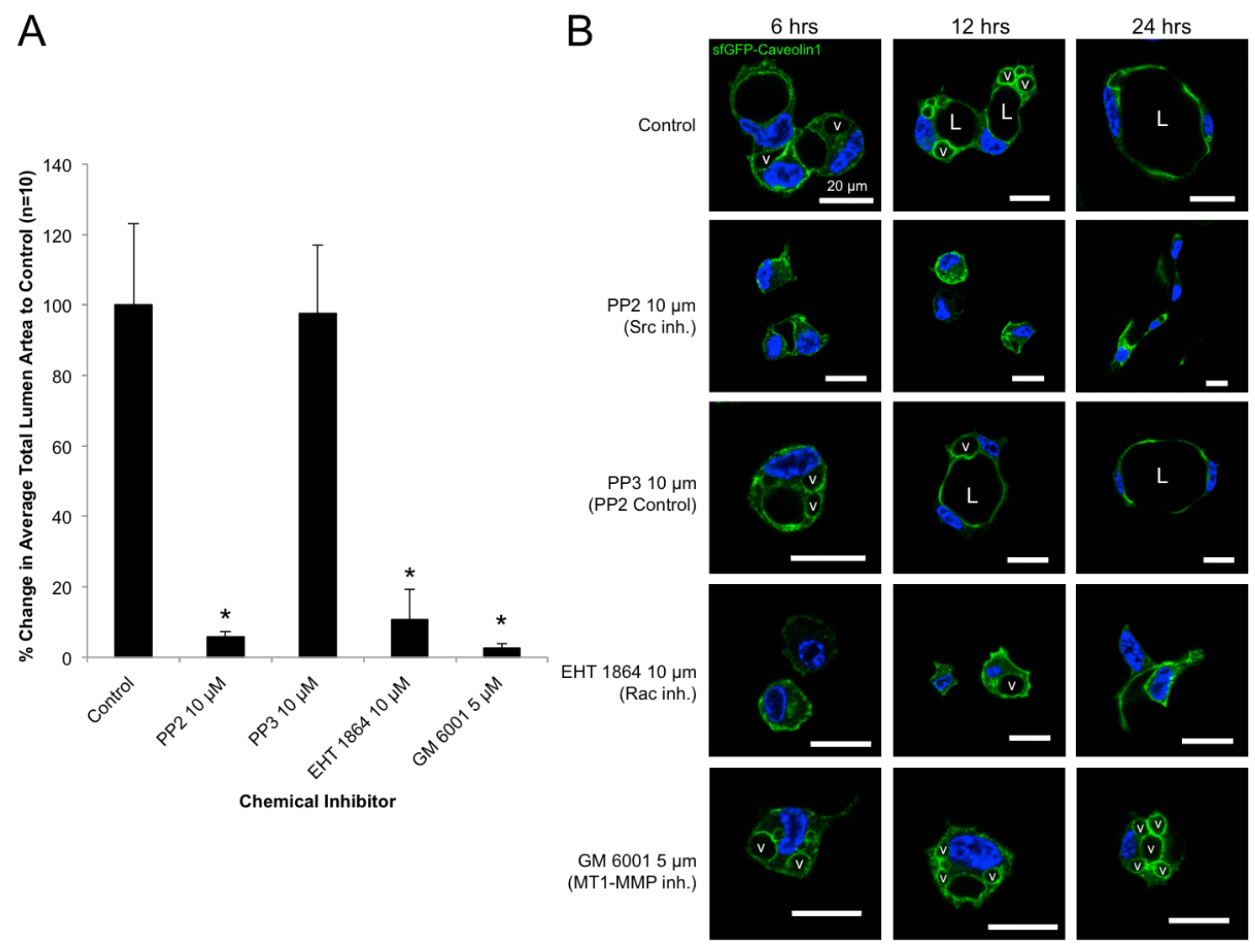

Figure 3.10. Src and Rac activity regulate pinocytic intracellular vacuolization necessary for EC tubulogenesis whereas MT1-MMP activity regulates fusion of intracellular vacuoles downstream. (A) Cultured ECs were harvested, suspended in 3D collagen matrices and fed with control culture media or media containing either PP2 10 $\mu \mathrm{M}$, PP3 $10 \mu \mathrm{M}$, EHT $186410 \mu \mathrm{M}$ or GM $60015 \mu \mathrm{M}$ and allowed to form vacuoles and lumens for $24 \mathrm{hr}$. before being fixed, stained, photographed and quantified for lumen area. Data are normalized to control samples and are reported as average lumen area per $\mathrm{HPF} \pm \mathrm{SD}(\mathrm{n}=10, \mathrm{p}<.01)$. Asterik indicates significance to control samples. (B) ECs cultures were infected with recombinant adenovirus carrying sfGFP-Caveolin 1 and treated with control culture media or culture media containing chemical inhibitors as in (A) and were allowed to form vacuoles and lumens over a period of 6-24 hr. Fixed cultures were then analyzed by confocal microscopy and representative images are shown. L indicates the EC lumen space and v indicates vacuoles. Bar equals $20 \mu \mathrm{m}$. 
FIGURE 3.11

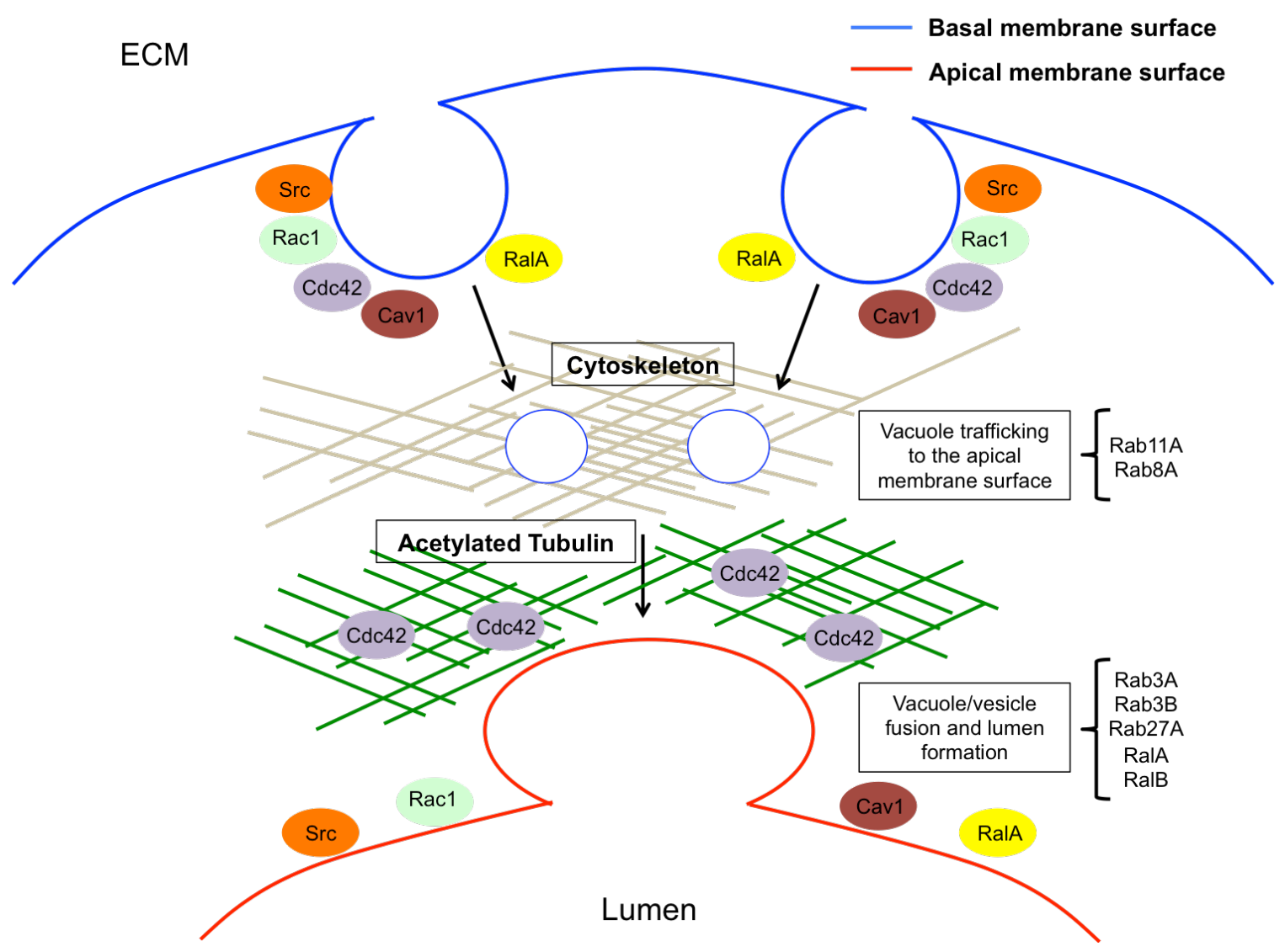

Figure 3.11. Generation, apical trafficking and fusion of pinocytic intracellular vacuoles along modified tubulin tracks is controlled by Cdc42, Racl, Rab and Ral GTPases in coordination with Src and Caveolin-1 during EC tubulogenesis. A schematic diagram is shown illustrating key steps in endothelial lumen formation differentially regulated by small GTPase, Src kinase and Caveolin-1 signaling. Pinocytic intracellular vacuoles containing Src, Rac1, Cdc42, Caveolin-1 and RalA are generated at the basal membrane surface where they are then trafficked apically by Rab11A and Rab8A regulated activity along acetylated tubulin tracks. Rab3A, Rab3B, Rab27A, RalA and RalB then regulate fusion of these vacuoles and other membrane vesicles to form the lumen. 


\section{CHAPTER IV}

\section{CONCLUSION}

In this work, molecular mechanisms of small GTPase regulation of asymmetric cytoskeletal polarization, polarized vacuole/vesicle trafficking and vesicle-to-vesicle fusion events during EC lumen formation and tubulogenesis are elucidated through use of 3D models of vasculogenesis under serum-free, defined conditions in type I collagen matrices. These assays have allowed for careful analysis of distinct signaling pathways regulated by GTPase activity to control early events of vasculogenesis as well as the spatiotemporal activity of key molecules involved in this process. Much of the work presented here is focused on the identification of new GTPases, critical downstream effectors and upstream regulators of GTPase activity controlling these events. In this way, we are able to begin to dissect key signaling pathways involved in asymmetric cytoskeletal polarization and lumen formation during EC tubulogenesis downstream of growth factor, integrin and matrix metalloproteinase signaling.

Previous work in our lab was the first to identify a necessary requirement for Cdc42 and Rac1 activity during EC tubulogenesis. We have now shown that Rac2, k-Ras and Rap1B activity are necessary during EC lumen formation and capillary assembly in addition to Cdc42 and Rac1 (Fig. 2.1). siRNA suppression of Cdc42 in combination with k-Ras, Rac2 and Rap1B resulted in the most profound blocking effects (Fig. 2.1) while suppression of key downstream effectors PAK2 and PAK4 in different combinations with one another or IQGAP1, MRCK $\beta, \beta$-Pix or Rasip1 resulted in similar effects (Fig. 2.6, 
Suppl. Fig. 2.2, Suppl. Fig. 2.3). Also, it was shown that increased expression of Cdc42, k-Ras particularly, and Rap1B stimulated EC tubulogenesis (Suppl. Fig. 2.1). We have also shown results from biochemical pulldown assays identifying multiprotein signaling complexes with Cdc42, Rac1, Rac2 and k-Ras with $\alpha 2$ integrin, MT1-MMP and key downstream effectors. Additionally, these signals were increased through increased expression of WT PKCE, correlating with stimulated EC morphogenesis and lumen formation (Fig. 2.2, Fig. 2.7). Thus, we have demonstrated that multiple GTPases and GTPase effectors work in concert with critical kinases such as Src, PKCE, Raf, MEK, and Erk to control EC tubulogenesis.

This notion is also supported by our findings showing that multiple GTPase activating proteins are responsible for negatively and positively regulating EC tubulogenesis. We have now shown that activation of endogenous Cdc42/Rac through siRNA suppression of Arhgap31, and k-Ras through suppression of Rasa1 stimulates EC tubulogenesis and increases vessel area whereas activation of endogenous RhoA by suppression of Arhgap29 inhibits EC tubulogenesis and impairs lumen formation (Fig. 2.4). Importantly, activation of $\mathrm{Cdc} 42$, Rac and k-Ras activity is synergistic and stimulates key downstream signaling pathways controlling EC tubulogenesis, such as PAK, ERK and other tyrosine kinase activity and tubulin modifications (Fig. 2.2, Fig. 2.4, Fig. 2.5). In contrast, activation of RhoA inhibits signaling through these pathways (Fig. 2.5) suggesting that a critical balance of signaling activity regulated by GTPases must be maintained during EC tubulogenesis to form a functional vasculature.

This work also demonstrates the critical role of the key GTPases and effectors in controlling EC asymmetric polarization during tubulogenesis. Through use of 
recombinant adenovirus reagents and immunostaining techniques, we showed that Cdc42, Rac, k-Ras and phospho-c-Raf accumulate at the developing apical membrane (Fig. 2.3, Fig. 2.6, Suppl. Fig. 2.1) and additionally show Rasip1's ability to target to the apical membrane surface both in vitro and in vivo (Fig. 2.6). We have also shown evidence that asymmetric polarization of cytoskeletal components by the subapical distribution of modified tubulins (particularly acetylated tubulin) and basal distribution of F-actin is necessary to direct membrane trafficking of vacuoles/vesicles to the nascent apical luminal membrane as vacuoles were observed to accumulate around regions of highly localized acetylated tubulin (Fig. 2.3). Finally, we have shown that acetylated tubulin and $\mathrm{Cdc} 42$ are highly co-localized at the subapical domain and demonstrated that the signaling activity of $\mathrm{Cdc} 42$, Rac, Ras, PAK and RhoA regulate these critical tubulin modifications during EC tubulogenesis (Fig. 2.3, Fig. 2.5, Suppl. Fig. 2.2). In this way, the activity of several GTPase signaling pathways in coordination with one another are critical in regulating events that control EC tubulogenesis (Fig. 4.1).

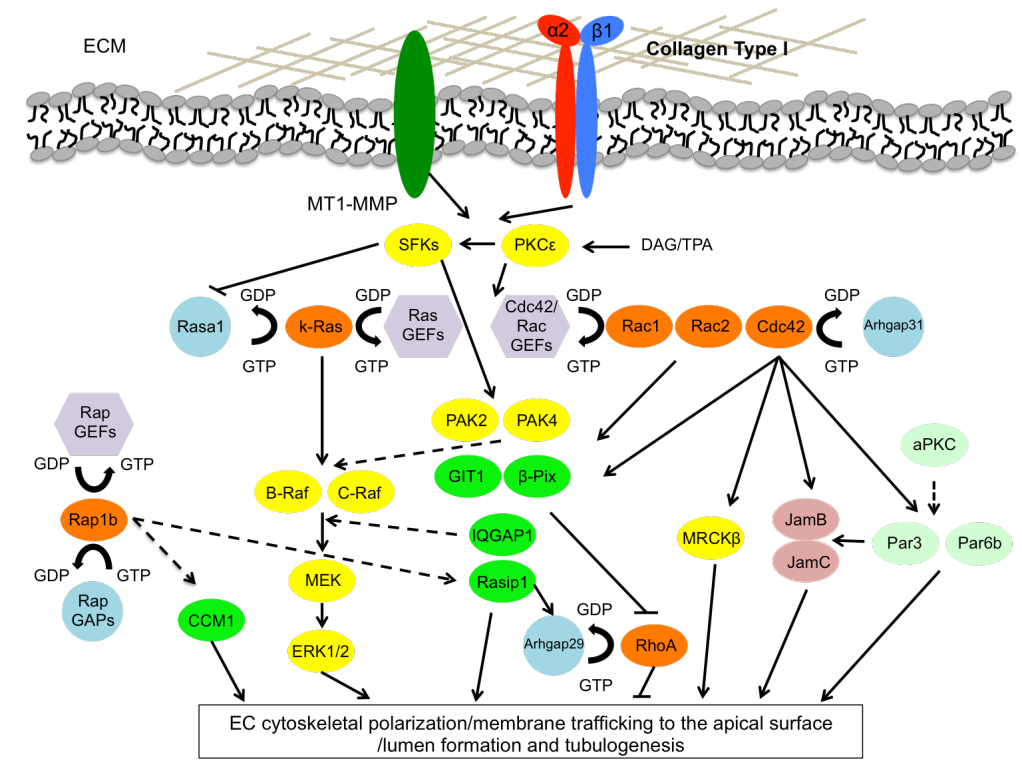


Figure 4.1. This schematic illustrates key molecular mechanisms regulated by GTPase activity controlling the different events in EC lumen formation and tubulogenesis.

Fundamental to EC lumen formation is the polarized trafficking of vacuoles and vesicles to the site of the developing apical luminal membrane from the basal membrane surface. Through work presented in this thesis, we identified novel roles for Rab3A, Rab3B, Rab8A, Rab11A, Rab27A, RalA and RalB small GTPases and Caveolin-1 in controlling endocytic membrane trafficking and exocytic vesicle fusion events (Fig. 3.1, Fig. 3.4). In contrast, siRNA suppression of the endocytic regulator Rab5A did not impair EC morphogenesis suggesting that Rab5A does not regulate macripinocytic mechanisms involved in EC tubulogenesis nor is EC lumen formation dependent on clathrin-mediated endocytosis (Fig. 3.1). In continued support of the conclusion that these GTPases possess different functional roles during the different stages of EC lumen formation, we observed greater blockade of EC tubulogenesis when combinations of siRNAs were used, such as Rab3A and Rab3B, Rab8A and Rab11A, RalA and RalB, and Rab3A and Rab8A (Fig.

\section{2, Fig. 3.3, Fig. 3.5, Fig. 3.6).}

Confocal microscopy analysis of the subcellular localization of several of these molecules during EC tubulogenesis demonstrated that they control EC polarization through distinct mechanisms. Through use of different combinations of recombinant adenovirus reagents carrying fluorescent fusion-proteins, we have shown that Caveolin1and RalA strongly target to intracellular vacuoles and the apical membrane and subapical domain as vacuoles coalesce (Fig. 3.7, Fig 3.8). However, Cdc42 and Rab11A are localized in a perinuclear, subapical region where vacuoles accumulate around (Fig 
3.7, Fig. 3.8, Fig. 3.9), similar to the distribution of acetylated tubulin (Fig. 2.3) suggesting that $\mathrm{Cdc} 42$ and Rab11 A positive vacuoles/vesicles migrate along modified tubulin tracks. Interestingly, we also observed that Src targets to intracellular vacuoles (Fig. 3.9) and its functional requirement along with Rac in controlling pinocytic vacuolization is supported through blockade of lumen formation and the reduction of vacuoles by use of chemical inhibitors (Fig. 3.10). Furthermore, we have shown a novel function for MT1-MMP activity downstream of intracellular vacuolization as blockade of its activity inhibited fusion of vacuoles/vesicles during EC lumen formation (Fig. 3.10).

The studies presented in this thesis represent a novel assessment of the molecular mechanisms controlling GTPase regulation during EC tubulogenesis, and are focused on delineating the separate steps involved in this process (Fig. 4.2). Through consideration of the findings presented here, it is of great importance in the vascular biology field to continue to investigate the highly coordinated mechanisms controlling EC polarization and lumen formation. In particular, the molecular mechanisms of EC tubulogenesis controlled by growth factor, integrin, MT1-MMP, Src family kinase and PKC $\varepsilon$ activity to regulate the downstream signaling pathways controlled by the GTPases identified in this work are critical to understanding blood vessel development and maturation, as well as what roles these signaling pathways may have in the context of vascular disease states. 


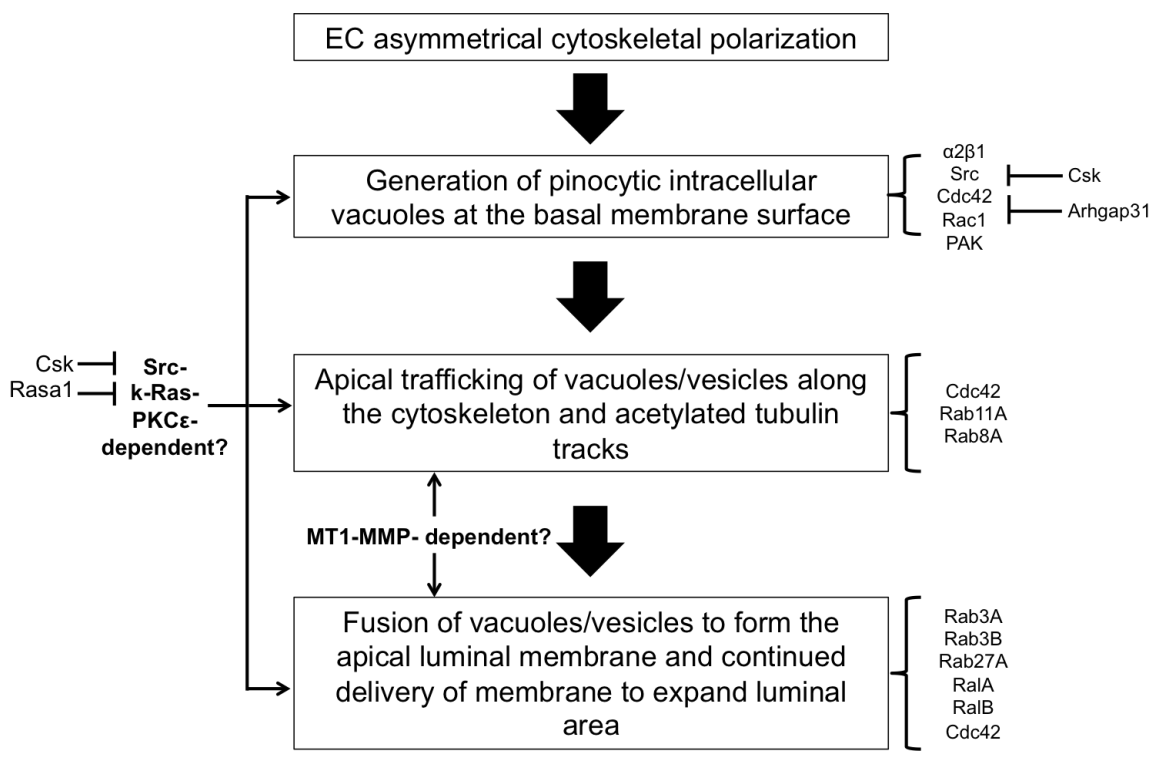

Figure 4.2. A schematic representation of the ideas and findings presented in this work, highlighting GTPase regulation of different steps in EC lumen formation in coordination with key kinase and integrin activity. 


\section{VITA}

Pieter Russell Norden was born August 16, 1989 in Columbus, Ohio. He graduated from Dublin Scioto High School in June 2007. He received a B.S. majoring in Biology and Chemistry from Hope College in Holland, Michigan in May 2011. In August 2011 he began working on his PhD in the Genetics Area Program at the University of Missouri-Columbia and continued his thesis work with Dr. George E. Davis in March 2012. During this time, he was awarded a Life Sciences Fellowship from the Univeristy of Missouri-Columbia to support his research. Following graduation he will pursue career opportunities in Chicago, Illinois. 